Portland State University

PDXScholar

Fall 12-18-2013

\title{
Battery Energy Storage Systems to Mitigate the Variability of Photovoltaic Power Generation
}

Heath Alan Gurganus

Portland State University

Follow this and additional works at: https://pdxscholar.library.pdx.edu/open_access_etds

Part of the Power and Energy Commons

Let us know how access to this document benefits you.

\section{Recommended Citation}

Gurganus, Heath Alan, "Battery Energy Storage Systems to Mitigate the Variability of Photovoltaic Power Generation" (2013). Dissertations and Theses. Paper 1495.

https://doi.org/10.15760/etd.1494

This Thesis is brought to you for free and open access. It has been accepted for inclusion in Dissertations and Theses by an authorized administrator of PDXScholar. Please contact us if we can make this document more accessible: pdxscholar@pdx.edu. 
Battery Energy Storage Systems to Mitigate the Variability of Photovoltaic Power Generation

\author{
by \\ Heath Alan Gurganus
}

A thesis submitted in partial fulfillment of the requirements for the degree of

\author{
Master of Science \\ in \\ Systems Science
}

Thesis Committee:

Wayne Wakeland, Chair

Robert B. Bass

Timothy R. Anderson

Portland State University

2013 
(c) 2013 Heath Alan Gurganus 


\begin{abstract}
Methods of generating renewable energy such as through solar photovoltaic (PV) cells and wind turbines offer great promise in terms of a reduced carbon footprint and overall impact on the environment. However, these methods also share the attribute of being highly stochastic, meaning they are variable in such a way that is difficult to forecast with sufficient accuracy. While solar power currently constitutes a small amount of generating potential in most regions, the cost of photovoltaics continues to decline and a trend has emerged to build larger PV plants than was once feasible. This has brought the matter of increased variability to the forefront of research in the industry. Energy storage has been proposed as a means of mitigating this increased variability - and thus reducing the need to utilize traditional spinning reserves — as well as offering auxiliary grid services such as peak-shifting and frequency control. This thesis addresses the feasibility of using electrochemical storage methods (i.e. batteries) to decrease the ramp rates of PV power plants. By building a simulation of a grid-connected PV array and a typical Battery Energy Storage System (BESS) in the NetLogo simulation environment, I have created a parameterized tool that can be tailored to describe almost any potential PV setup. This thesis describes the design and function of this model, and makes a case for the accuracy of its measurements by comparing its simulated output to that of well-documented real world sites. Finally, a set of recommendations for the design and operational parameters of such a system are then put forth based on the results of several experiments performed using this model.
\end{abstract}




\section{Dedication}

I would like to thank the many people who played either direct or indirect roles in making this thesis possible. My friends, family, and coworkers have all contributed in ways large and small - most often simply having a level of patience and understanding uncommon to most people. Most of all I would like to thank my wife, Jordan, whose ceaseless support and encouragement from day one of graduate school up to the last night of writing has enabled me to focus the entirety of my efforts on this one endeavor, and I will be forever grateful to her for that. I dedicate this thesis to her. 


\section{Acknowledgements}

I would like to acknowledge the generous help and support of my thesis committee members, Dr. Robert Bass and Dr. Timothy Anderson, and my thesis committee chair, Dr. Wayne Wakeland. This thesis is in no small part a product of their insight and thoughtful feedback throughout the process, and I thank them for that. I would also like to thank the faculty and staff of the Systems Science program for their guidance throughout my graduate career. Finally I would like to thank my peers in the Systems Science program for making me feel completely at home in Portland, and for always finding beauty in the complexity that is the world around us. 


\section{Contents}

Abstract

Dedication $\quad$ ii

Acknowledgements

List of Tables vii

List of Figures $\quad$ viii

1 Introduction and Background 1

1.1 Introduction . . . . . . . . . . . . . . . . . . . . 1

1.1.1 Wind and Solar Power . . . . . . . . . . . . . . . . . . 3

1.1.2 Ramp Rates . . . . . . . . . . . . . . . . . . . 4

1.1.3 Energy Storage . . . . . . . . . . . . . . . . . . 6

1.2 Anticipated Contribution . . . . . . . . . . . . . . 8

1.2.1 Research Question ................. . 9

2 Research Methods 10

2.1 NetLogo Model . . . . . . . . . . . . . . . . . . . . . . . . . 10

2.1 .1 Solar Irradiance . . . . . . . . . . . . . . . . . . . . . . . 13

2.1.2 Photovoltaic Power Generation . . . . . . . . . . . . 20

2.1.3 Battery Energy Storage System . . . . . . . . . . . . . . . 21

2.2 Model Testing . . . . . . . . . . . . . . . . . . . . . 31

2.2.1 Comparison with NREL MIDC Data . . . . . . . . . 31

2.2.2 Comparison with UO SRML Data . . . . . . . . . . . . 39

2.2.3 Model Parameters . . . . . . . . . . . . . . . . . . . 40

2.3 Search for Reasonable Configuration of Battery Parameters . . . . . 43

2.3.1 Absolute Leveling . . . . . . . . . . . . . . . . . . . . . 44 
2.3.2 Reactive Leveling . . . . . . . . . . . . . . . . . . . 45

2.3 .3 Time-Shifting . . . . . . . . . . . . . . . . . 46

$\begin{array}{lll}3 & \text { Results } & 47\end{array}$

3.1 BehaviorSpace Experiments . . . . . . . . . . . . . . . . 47

$3.1 .1 \quad$ Experiment $1 \ldots \ldots \ldots \ldots$

3.1 .2 Experiment $2 \ldots \ldots \ldots \ldots$

3.1 .3 Experiment $3 \ldots \ldots \ldots \ldots \ldots$

3.1 .4 Experiment $4 \ldots \ldots \ldots \ldots \ldots$

3.1.5 Experiment $5 \ldots \ldots \ldots \ldots \ldots$

3.1.6 Experiments 6, 7, and $8 \ldots \ldots \ldots \ldots$

3.1 .7 Experiment $9 \ldots \ldots \ldots 1 \ldots \ldots \ldots$

3.1 .8 Experiment $10 \ldots \ldots \ldots 6 . \ldots \ldots$

3.1 .9 Experiment $11 \ldots \ldots \ldots 2 \ldots \ldots . \ldots \ldots$

4 Discussion and Conclusion $\quad 65$

4.1 Analysis and Interpretation of Primary Results . . . . . . . . . . 65

4.1.1 Optimal Battery Chemistry . . . . . . . . . . 65

4.1.2 Feasible Steady State Charge Based on Predicted Variability 66

4.1.3 BESS Energy Capacity and Leveling Target . . . . . . . . 67

4.1.4 Optimality and Robustness . . . . . . . . . . . 71

4.2 Limitations and Opportunities for Future Improvement . . . . . . . 73

4.3 Effect of PV Dispersal Area on Average Variability . . . . . . . . . 75

4.4 Role of Infrastructure Integration . . . . . . . . . . . . . . . . 78

4.5 Conclusion . . . . . . . . . . . . . . . . . . . . . . 79

$\begin{array}{lr}\text { Bibliography } & 82\end{array}$

$\begin{array}{ll}\text { Appendix A: Supplemental Data } & 91\end{array}$

A.1 Energy Price per kWh . . . . . . . . . . . . . . . . . . 91

A.2 Initial Model Versions _. . . . . . . . . . . . . . . . 91

A.3 Preliminary Results . . . . . . . . . . . . . . . . . . . . . 92 
A.4 Experiment 5 Parts I \& II . . . . . . . . . . . . . . . . . 92

$\begin{array}{ll}\text { Appendix B: Data Processing } & 94\end{array}$

B.1 FileIO Script . . . . . . . . . . . . . . . . . . 94

B.2 Shell Script . . . . . . . . . . . . . . . . . . . 97 
List of Tables

2.1 Battery Types and Their Attributes . . . . . . . . . . . . . . 29

2.2 Sensitivity Table for Model Parameters . . . . . . . . . . . . . . . . 42

3.1 Lowest Variability Configurations from BehaviorSpace Experiment 149

3.2 Lowest Variability Configurations from BehaviorSpace Experiment 251

3.3 Lowest Variability Configurations from BehaviorSpace Experiment 356

3.4 Lowest Variability Configurations from BehaviorSpace Experiment 458

3.5 Lowest Variability Configurations from BehaviorSpace Experiment 560 


\section{List of Figures}

2.1 User Interface of the BESS Model in NetLogo . . . . . . . . . . . . 11

2.2 Preliminary Design of BESS Model . . . . . . . . . . . . . . . . . . 22

2.3 Map of Module Placement at NREL Oahu Site . . . . . . . . . . . . 32

2.4 Simulated and Observed Peak and Average Irradiance (NREL Data) 36

2.5 Simulated and Observed Peak Variability (NREL Data) . . . . . . . 37

2.6 Simulated and Observed Average Variability (NREL Data) . . . . . 38

2.7 Simulated and Observed Daily Irradiance (SRML Data) . . . . . . . 40

2.8 Simulated and Observed Monthly Irradiance (SRML Data) . . . . . 41

2.9 Simulated and Observed Average Variability (SRML Data) . . . . . 43

3.1 Output of an Optimal Configuration from Experiment 1 . . . . . . 49

3.2 Output of an Optimal Configuration from Experiment 2 . . . . . 51

3.3 Output of an Optimal Configuration from Experiment 3 . . . . . 57

3.4 Output of an Optimal Configuration from Experiment 4 . . . . . 58

3.5 Output of an Optimal Configuration from Experiment 5 . . . . . 60

3.6 Results of BehaviorSpace Experiment 9 . . . . . . . . . . . . . 61

3.7 Results of Experiment 9 for NaS_15 Type . . . . . . . . . . . . . . 62

3.8 Results of BehaviorSpace Experiment 11 . . . . . . . . . . . 63

3.9 Results of Experiment 11 for Vanadium Type . . . . . . . . . . . . 64

4.1 Suboptimal Output of an Optimal Configuration from Exp. 2 . . . 72

4.2 Map of Regions of Interest at NREL Oahu Site . . . . . . . . . . . 76 


\section{Introduction and Background}

\section{$1.1 \quad$ Introduction}

Since the beginning of the industrial revolution, the scale and complexity of human technology have increased greatly; since the beginning of the information age the pace of this increase has quickened exponentially. Modern technological advancement has, by most accounts, improved both the length and quality of human life in most places of the civilized world. Advances in medicine and healthcare have eliminated many diseases that just a hundred years ago decimated entire populations. Advances in communication have not only brought together peoples of disparate societies and improved understanding between them, but have also given those in oppressed cultures the means to unify their voices and overcome the obstacles that would otherwise hinder them from living fulfilled, prosperous lives. Advances in physics have increased the scale of the universe that is known to us by a factor of $3.8 \times 10^{5}$, while also revealing scales $3 \times 10^{5}$ smaller than what was a hundred years ago deemed to constitute the "basic building blocks" of the universe. Advances in travel in the past century have taken us from the age of horse power, which had persisted since the dawn of civilization thousands of years ago, to the age of space travel - not to mention intercontinental terrestrial travel in a twentieth of the time required in the year 1900 [1].

One of the most unifying attributes of all this advancement, however, is the need for enormous amounts of energy. Many of the advancements of the twentieth century involved the ability to harness ever greater amounts of energy — but that energy must first be produced. The vast majority of the energy that was produced 
in the past century originated from non-renewable sources, including hydrocarbon sources such as coal, oil, and natural gas. Coal and natural gas continue to dominate power generation because of their low cost and high level of dispatchability which means that their output can be adjusted on demand. However, there are myriad reasons why continued reliance on these sources will be unwise. There are many well-documented health and public safety concerns with the procurement, transport, and combustion of these fuels [2]. Furthermore, it has been shown that the carbon dioxide emitted from hydrocarbon fuel sources is contributing to the warming of our atmosphere through the greenhouse effect [3]. Strategically, placing such a high dependence on a fuel source such as petroleum that many countries must import from foreign nations places them at a disadvantage in international trade negotiations.

Nuclear power, discovered halfway through the last century, has contributed a great deal toward our understanding of low-emissions power generation. However, the fuel used for generating power through nuclear fusion, uranium-235, is considered non-renewable [4]. There is also an ongoing public concern over the inherent risks of radiation leaks from a nuclear core overheating [5]. While these risks may be effectively mitigated in the future, work remains to reengineer the necessary failsafe mechanisms. Furthermore, there are serious concerns about the ability of any man-made system to effectively contain the nuclear waste that is generated through nuclear power generation — especially when one considers the timescales that are involved in the half-life of spent nuclear fuel.

Some forms of power generation based on renewable sources such as biofuels may be effective in reducing carbon dioxide emissions, and may offer the ability to be "regrown" and thus not easily depleted, yet may be unsustainable on a large scale for other reasons. Foremost of these is the possible connection between biofuel demand and global food prices [6]. 
Hydroelectric power offers a host of benefits including dispatchability, potential scalability, low-cost, sustainability, and lack of detrimental carbon dioxide emissions. In fact, in several regions of the world hydroelectric sources constitute the majority of locally-sourced power. The limiting factor with hydro power is its highly localized nature. The regions that benefit most from hydro power, such as the northwestern United States, have abundant supplies of quickly moving water - as hydropower potential is a product of hydraulic head (i.e. the liquid surface elevation) and the rate of flow of the water. In areas of the world where adequate levels of each of these measures is not attainable either naturally or through man-made structures, hydroelectric generation is simply not practical.

\subsubsection{Wind and Solar Power}

Finally there is electric power generated from wind and solar energy. The methods utilizing these energies include wind turbines that harness the mechanical force of the wind, solar thermal facilities that use the Sun's energy to heat a liquid to power a steam generator, and solar photovoltaic (PV) arrays, which use the excitation energy from the Sun's light rays to create an electric potential across a series of semiconductors - turning sunlight directly into DC electrical power.

The benefits from these power sources are numerous and distinct. Their "fuel", (i.e. wind and sunlight), is not physically harvested in the traditional sense, and there is no waste byproduct due to generation, so there is minimal impact on the environment relative to the mining, drilling, refining, and transport required of other fuels. In general, while any endeavor of advanced technology in the modern world is not going to be without some kind of effect on the natural world, the most widely perceived negative effects of solar and wind energy are indeed an extreme improvement over the use of fossil fuels for power generation. The two prominent issues raised with wind power in particular are in regard to their effects on bird 
population [7], and their relationship to a host of ill-defined and almost universally reputed maladies to human health. Studies have shown that the reported health effects of wind farms are most likely based on one's perceived expectation of health effects [8] or a physical manifestation of one's attitudes on the subject of wind energy in general [9].

In regard to the effect of wind farms on bird populations, it has been shown that while there is a measurable negative effect, this effect can be minimized by following certain siting and operational guidelines [7]. Furthermore, it has been shown that the measured negative effects to bird populations due to wind farms are negligible both in magnitude and scope relative to the negative effects to bird populations due to hydrocarbon-based power generation [10, 11, 12].

The primary negative effects of solar arrays are found in the manufacturing of the PV panels. While a substantial amount of energy is used to build them, this is offset by 9-17 times that much energy being generated over the lifetime of the panel [13]. The main perceived concerns relate to the materials used during this process - including sustainable procurement of scarce Earth elements, air and water pollution from the waste materials, and disposal of the module after the end of its operational life. While there certainly remains room for improvement in these areas, enormous improvements have already been made in the past 5-10 years as new operational practices have been discovered and implemented. Furthermore, by similar logic to that used with wind energy, the concerns associated with PV production and use are minimal compared with those faced by continued reliance on fossil fuels for energy generation $[13,14,15,16,17]$.

\subsubsection{Ramp Rates}

The primary fundamental limiting factor for wind and solar PV power is that they are both highly stochastic, albeit on different timescales. Wind farm ramp 
rates are often more manageable in engineering terms than solar PV ramp rates. An average maximum ramp rate for a wind farm is about $2 \%$ per minute, while that of solar PV facilities can be much higher - up to $70 \%$ per second [18]. This is due to the fact that changes in wind speed are normally less sudden and less drastic than changes in solar radiation intensity due to passing cloud cover, as well as the fact that the mechanical operation of wind turbines affords it momentum that tends to mitigate small scale drops in wind speed. There are no such moving parts to provide momentum in PV generation. The ramp rates of solar thermal plants are less than that of solar PV arrays because they possess both thermal and mechanical inertia that the PV plants do not. However, the ramp rates of solar thermal will still be substantially greater than that of wind plants.

Because of the need for the generation and load of the electric grid to be exactly in sync, the loss of wind or solar power must be mitigated elsewhere by more traditional generation methods; and the intensity of the ramp rate necessitates that those facilities "spin-up" in a way that is both inefficient and expensive [19]. Furthermore, even this approach has its limitations, and any decrease in the renewable generation on the grid that exceeds the ability of reserve facilities to spin-up could create blackout scenarios. The maximum rate of change of a facility's output to the grid (i.e. its ramp rate) is determined by the NERC control criteria and is based on both the availability of standby generation on the grid interconnect as well as the mechanical properties of the switching mechanisms at the local substation. These switching mechanisms have stated operational lifetimes that can be reduced precipitously by highly variable power generation. Because of the stochastic nature of wind and solar energy, it has been said that their integration into the overall electric grid cannot surpass $35 \%$ without creating reliability issues $[20,21]$. 


\subsubsection{Energy Storage}

There are many methods that have been proposed to mitigate the variability of these stochastic sources, and one of the most promising is that of energy storage. Energy storage of various kinds has been around since prehistoric times. Since the advent of electrical power, energy has typically been stored in the form of fuel, and then converted to electricity as needed. By far the most common form of this today is pumped hydro, in which water is pumped up an elevation to a reservoir during times of low power demand, and allowed to flow back downhill through hydroelectric generators during time of high power demand. This method, while relatively efficient, affordable, and scalable, has the same fundamental limiting factor as hydroelectric generation at large which is that it is not viable in places without both an abundant supply of water and a high topographical gradient.

Among the other forms of energy storage, including flywheels, supercapacitors, and compressed air storage, electrochemical energy storage in the form of batteries has the most promise for mitigating short to long-term variability in wind and solar power generation because batteries have a high level of efficiency, low response time, and dispatchability. The cost and limited service life of batteries continues to restrict their mainstream implementation, but these factors are changing because of the lowering costs of battery technology from new chemical compositions, as well as evolutionary improvements in existing compositions. As numerous civil bodies establish goals for the percentage of their energy generated from renewables, the need grows to alleviate concerns of the reliability of these sources. Batteries offer the speed, reliability, and dispatchability to directly address these issues.

There are many different concerns that may arise as batteries are integrated into power generation systems. This thesis addresses some of the most basic and am- 
biguous of these in a comprehensive and holistic manner. In doing so, I outline the probable benefits of several different configuration choices for these storage systems, so that utilities and project designers have a clearer indication of the capabilities offered by the inclusion of such a system. I have taken a systems approach to problems that are somewhat beyond the scope of electrical engineering in the strictest sense, in the hope of bringing perspective to the higher-level design choices. In particular, I have attempted to define a battery storage system as the completion of a complete energy system. A rigorous definition of this system is given in Section 2.1.

Photovoltaic (PV) solar arrays have ramp rates that are the highest of the generation methods listed - though typically the decreases in solar radiation due to cloud cover are much shorter lived than decreases in wind speed. Furthermore, they generally have an overall generation curve that is much easier to predict, (i.e. the length of a solar day). The quick response times of batteries are an ideal match for the second-to-second variability of PV farms, while the short duration of PV variability necessitates only a minimum energy storage capacity — which would otherwise significantly increase the cost of battery systems. For this reason battery energy storage systems are relatively better suited for PV plants than wind generation projects.

The goal of this thesis is to determine the ideal configuration of a battery energy storage system (BESS) to suit the given priorities and goals of an entire utility or those of an individual project, including finding the most feasible settings for parameters determining:

- What battery type to use

- How much energy storage capacity they should maintain

- How much energy to store initially each day before outputting to the grid

- How much variability should the system attempt to mitigate 


\subsection{Anticipated Contribution}

In general, the goal of this thesis is to deepen our understanding of what is possible in terms of the mitigation of variability in renewable power generation through energy storage. Specifically, this thesis aims to determine the reasonable configurations of a potential energy storage system to mitigate variability and increase revenue for a given PV power plant, by modeling, simulating, and analyzing the output of a standard battery energy storage system.

It is important to understand the limitations of the study, as well. It is not intended to address issues such as reactive power and frequency response - mostly because this would necessitate circuit-level modeling that would increase the complexity and runtime of the simulation considerably, without increasing the information pertaining to the core feasibility of the system substantially. A manageable runtime was important in making adequate scope and granularity in the results practical.

Likewise, this thesis is not meant to be interpreted as a cost-benefit analysis of a BESS system. While certain feasibility measures are based on estimated initial and operating costs, these measures are not sufficient to address the overall cost effectiveness of energy storage for every system. While the cost of implementing battery storage for a particular project will be relatively consistent across a geographic region, the return on this investment will vary considerably, based on the overall rate of energy and the premium paid for consistency in generation. Finally, state and local incentives vary widely from one potential site to another, and are not easily modeled in a systematic way. 


\subsubsection{Research Question}

Ultimately, the model described in this thesis is meant to supplement, rather than replace, existing tools being used in the industry and to provide a resource for those looking for comprehensiveness and parameterization. This thesis is meant to describe the workings of the model in sufficient detail, to verify the output of the model against reliable irradiance observations, and to find the configurations of a potential BESS that would result in desirable system output when coupled with a given PV plant. The primary research question throughout the process has been whether a BESS would be feasible under the circumstances seen by most PV projects, and how such a BESS should be configured to enable that feasibility. 


\section{Research Methods}

The general method of research for this thesis was to build a model of a PV plant with an integrated BESS and observe how such a storage system could be used to reduce the variability of the plant's output. This system was modeled in NetLogo, and then its simulated irradiance was verified against observed irradiance using data from the National Renewable Energy Laboratory (NREL) Measurement and Information Data Center (MIDC), and the University of Oregon Solar Radiation Monitoring Laboratory (SRML). Finally, I performed experiments with different configurations of the BESS model to find desirable output characteristics based on certain user-defined system goals, such as leveling the output of the system. I analyzed the results of these tests and outlined a summary of the anticipated practicality of such a system under several different common scenarios.

\subsection{NetLogo Model}

The model was designed to address the needs of the present research and also to be used as a tool by utility companies and project managers. With that in mind, I wanted to build the model in an environment with a capability for robust calculation and runtime performance, while also including a reasonably simple user interface so that there would be relatively less of a software-specific learning curve compared with other software packages or command line tools. NetLogo was chosen as the simulation environment of choice for these reasons. The user interface of the model is shown in Figure 2.1. 

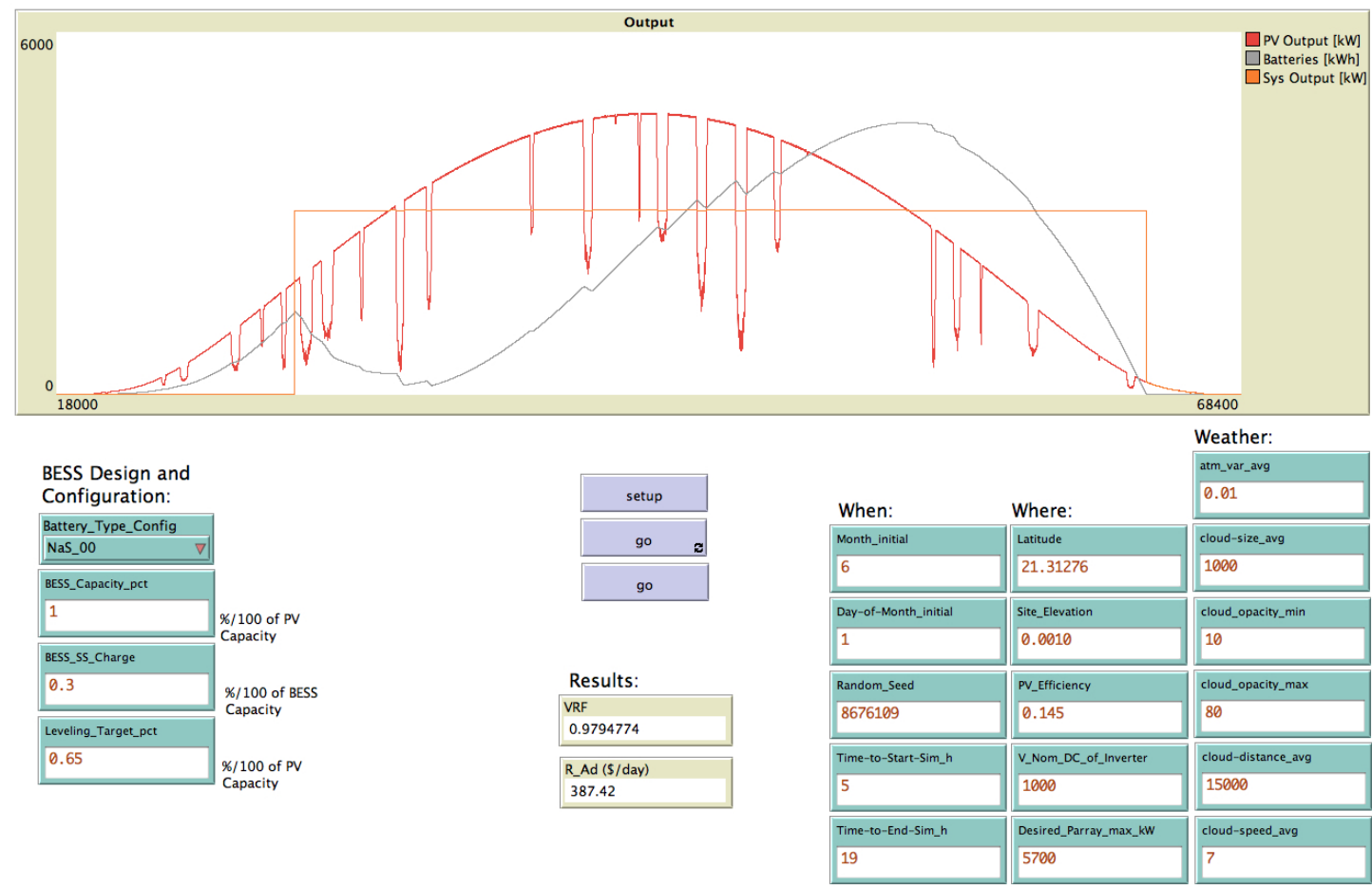

Figure 2.1: The user interface of the BESS model in NetLogo, shown with simulated output for a particular day in June

The general design of the model is based on a series of variables and parameters as follows ${ }^{1}$ :

- When and Where

1. Month

2. Day of Month

3. Latitude

4. Site Elevation

- PV Array

1. PV Area

2. PV Efficiency

- Weather Characteristics

1. Average Atmospheric Variability (cloudless)

2. Average Cloud Size

3. Minimum Cloud Opacity

\footnotetext{
${ }^{1}$ Note that not all variables listed here are implemented in the UI of the model - some less frequently used variables are set in the code of the model. Furthermore, some parameters visible in the UI lead to the calculation of the variables listed here, such as the PV efficiency, nominal DC voltage of the inverter, and the desired peak power of the array determining the number of modules to be used and the total array area.
} 
4. Maximum Cloud Opacity

5. Average Distance Between Clouds

6. Average Wind Speed

- BESS Configuration Parameters

1. Capacity

2. Minimum Charge

3. System Efficiency

4. Peak Charge Rate

5. Peak Discharge Rate

6. Discharge Time

7. Leveling Target

\section{General Operation}

After entering the values for these parameters, (which are pre-populated with default values), the user first clicks the 'Setup' button, and then the 'Go' button. When the Setup button is clicked, a number of calculations are carried out by the model that $A$ ) are useful to display to the user before the simulation begins, such as the ramp rate limit and the forecasted peak system output or $B$ ) are necessary for the calculation of the rest of the model and need not be carried out at each step in the simulation. Examples of this latter type include the solar flux upon the outer atmosphere of the Earth from space, and the solar declination - neither of which change over the course of a day.

When the Go button is clicked, the simulation begins by counting off ticks, which can be set equal to any timescale desired. One tick is set equal to one second for the sake of simplicity in this model. The entirety of the code of the model (outside the Setup) is contained within this Go process. The model loops through further iterative ticks of this command until a preconfigured stopping point, at which time the model stops and awaits user input. In a general sense, the model follows the following outline during the Go command:

1. The solar energy at the top of the atmosphere is calculated 
2. The cloud cover and atmospheric variability is calculated

3. These effects are applied to the irradiance to find the energy incident upon the PV array

4. A section of charge/discharge logic determines when the BESS charges and discharges for each given application

5. The Go process ends, and then restarts at exactly one "tick" later

The results of these runs are shown as output on a plot in the UI window, and can be saved as txt or csv files for data analysis in other programs.

The model follows a modular design for debugging purposes and to allow for easy expansion and added accuracy in the future. It consists of three components: Solar Irradiance, Photovoltaic Power Generation, and the Battery Energy Storage System.

\subsubsection{Solar Irradiance}

The model includes the applicable inputs, systemic variables, terminal outputs, and internal logic of the system as a whole. The first, most basic input is incident solar radiation, hereafter referred to as solar irradiance. Irradiance is a measure of the power incident on a surface, and is measured in units of $W \cdot m^{-2}$. The solar irradiance of a site depends primarily on the latitude of the PV installation and the frequency, duration, and albedo of cloud cover [22]. When modeling the solar irradiance on the PV modules at ground level, I considered where the site would be, when the simulation would begin (i.e. date and time), and how it would be affected by the atmosphere. The first two variables were fairly straightforward and could be adequately summarized by the latitude of the installation and the datetime the simulation would begin to approximate with a high degree of accuracy the solar energy at the upper atmosphere. During the initial testing phase, the default setting of the model was to begin the simulation at 5 am and end it at 8 pm, simply because that is when the data from the Oahu station begins and ends. This is due to the fact that this array is in the tropics, and daylight never persists 
outside of these hours for the tropical latitudes [23, 24]. Here, "daylight" refers to the time between solar sunrise and sunset, which for Oahu on the Summer Solstice in 2010 was 13 hours and 27 minutes from 5:52 am to 7:18 pm [25]. This setting can be changed for scenarios outside of the tropics, when the length of the solar day would be longer in the summer, or for when a simulation over the course of more than a day would be desired to test the cyclical effects of energy storage.

The variables for latitude $(\phi)$ of the installation and the date to begin the simulation are then used to find the solar radiation at ground level in the absence of cloud cover. I began by finding the solar declination $\left(\delta_{\odot}\right)$, which is a measure of the seasonal variation of the Sun's position in the sky, and is outlined in [26]. This is given as

$\delta_{\odot}=\arcsin \left(\sin (-23.44) \cos \left(\frac{360}{365.24}(\right.\right.$ Date +10$)+\frac{360}{\pi} \cdot 0.0167 \cdot \sin \left(\frac{360}{365.24} \cdot(\right.$ Date -2$\left.\left.)\right)\right)$

The only user-supplied value in this equation is Date, which is the day number out of 365. Next the reduction of irradiance due to the air mass of the atmosphere was incorporated. The air mass factor Air_Mass represents the relative amount of atmosphere that sunlight must pass through to reach the ground at any given point in the day. In the formulation used in the model, this factor is representative only of the relative amount of atmosphere due to the position of the sun relative to the zenith. A separate absorption coefficient $k$ is used to account for the effect of the elevation of the site on the irradiance. The equation relating these factors to the irradiance is given further in Equation 2.10. The air mass factor Air_Mass is greatest at sunrise and sunset, and minimal at noon [27]. This equation is given as

$$
\text { Air_Mass }=\left(\cos \left(\theta_{\odot}+0.15 \cdot\left(93.885-\theta_{\odot}\right)^{-1.253}\right)^{-1}\right.
$$


where $\theta_{\odot}$ is the solar zenith angle, or the angle of the sun relative to if it were directly overhead $[28,29]$. This is given by the equation

$$
\theta_{\odot}=\arccos \left(\sin (\phi) \cdot \sin \left(\delta_{\odot}\right)+\cos (\phi) \cdot \cos \left(\delta_{\odot}\right) \cdot \cos (\text { Hour Angle })\right)
$$

where Hour Angle is given by

$$
\text { Hour Angle }=15 \cdot\left(\text { Time_h }_{-}-12\right)
$$

Furthermore, the solar altitude angle $\left(\alpha_{\odot}\right)$, or the angle between the center of the Sun's disk and the horizon, is given by

$$
\alpha_{\odot}=\arcsin \left(\sin (\phi) \cdot \sin \left(\delta_{\odot}\right)+\cos (\phi) \cdot \cos \left(\delta_{\odot}\right) \cdot \cos (\text { Hour Angle })\right)
$$

or, more simply

$$
\alpha_{\odot}=90^{\circ}-\theta_{\odot}
$$

These equations make some approximations. The first is that they are based on the geocentric, rather than geodetic latitude — which is related to the fact that the latitudes used approximate from the center of the Earth as a sphere, rather than from the equatorial plane as an ellipsoid. This alters the calculated value by not more than 12 arc minutes. The second approximation is that the equations ignore the effects of atmospheric refraction. Refraction causes the Sun's image to be shifted by 35.4 arc minutes at the horizon, down to 9.9 arc minutes at $5^{\circ} \alpha_{\odot}$, and then quickly dissipating to zero above this point $[30,31]$. Because the energy incident upon an average PV installation from sunlight refracted at these angles is so low, these approximations were foreseen to not have a major impact on the results of the model. 
The length of the solar day (LOD) was found by

$$
L O D=\frac{48}{360} \cdot \arccos \left(-\tan \left(\delta_{\odot}\right) \cdot \tan (\phi)\right)
$$

This was used as a secondary measure (with the solar zenith angle) to determine when the PV system should expect to begin to see solar irradiance.

An assessment was made regarding the inclusion of the accurate solar noon time for each day. It was concluded that this calculation - especially the equation of time - would add significantly to the complexity of the model without offering much in the way of increased model precision. For the model, the mean solar time (i.e. $12 \mathrm{pm}$ ) was used each day as the point that the sun would reach its highest point of the day. Because of this approximation, the simulated output is timeshifted by up to half an hour from the observed output. This was determined to not be too much of a detriment to the core utility of the model. Even in multi-day simulations, the extreme amount of capacity that would be required to maintain a charge above the minimum charge from one day to the next was not evaluated because that configuration would currently be unfeasible under almost any scenario.

The direct normal irradiance $\left(I_{D i r N}\right)$ is the amount of solar radiation directly incident on a surface normal to the Sun, (i.e. without first being reflected by air particles). This is the type of irradiance that is the most energetic, and forms the largest portion of any PV array's incident energy. It is typically measured by an instrument rotating on a two-axis tracking system to maintain a normal orientation to the Sun, while having a collection area with a narrow field of view of usually about 5 degrees. To find this, we would first calculate the solar flux at the outer atmosphere $\left(I_{o}\right)$ as given in [32] by the equation

$$
I_{o}=I_{S C} \cdot\left(1+0.0344 \cdot \cos \left(360 \cdot \frac{\text { Date }}{365}\right)\right)
$$


where $I_{S C}$ here is equal to the solar constant, which is $1.367 \mathrm{~kW} / m^{2}$ [33], and the date is the number of the day out of 365, (i.e. 121 for June 20). Then, to calculate $k$, which is an absorption constant equal to

$$
k=0.2711+0.01858 \cdot(2.5-h)^{2}
$$

where $h$ is the elevation of the site [33]. The variable $h$ is set to be equal to the parameter Site_Elevation in the initial setup of the model. Finally, $I_{D i r N}$ can be calculated for a clear day by $[32,34,35]$

$$
I_{D i r N}=I_{o} \cdot e^{\left(-k \cdot A i r_{-} M a s s\right)}
$$

There are several factors that affect the transmission of solar radiation through the Earth's atmosphere. The three most prominent are absorption by atmospheric gases and conversion to heat, Rayleigh scattering by atmospheric gases, and aerosol scattering by atmospheric particulate matter. Each of these will reduce the direct incident solar irradiance, yet the latter two are collectively referred to as diffuse irradiance. Because their energy is reflected by the atmospheric particles, rather than absorbed, some of it is reflected to the ground, and thus to the PV array. Although diffuse radiation can be incident upon a PV array from any point in the atmosphere above it, the amount of energy from diffuse radiation is small because of the fact that much of the reflected radiation is reflected toward space, and because each time the photons are reflected they lose energy. Therefore on clear days at peak capacity, diffuse irradiance usually accounts for $10 \%$ or less of overall power generation. On partly cloudy or overcast days, when the direct irradiance is greatly reduced, diffuse irradiance can make up the majority of the incident radiation - yet the overall output is still much less than that of clear days [32, 36, 37].

Diffuse normal irradiance $\left(I_{D i f f N}\right)$ is the solar radiation incident on a surface nor- 
mal to the direction of the Sun that has been refracted from atmospheric particles such as air molecules, water vapor, and particulate matter from pollution. It can typically be approximated as $I_{D i f f N}=.1 \cdot I_{D i r N}$ under most circumstances. This was later found to be an inadequately accurate approximation, and a formulation that more closely matched the observed data is outlined in Section 2.2.

When combined with direct normal irradiation, these constitute the global normal irradiation $\left(I_{G N}\right)$ - which is the overall amount of power that would be incident on an array of PV panels mounted on two-axis tracking systems to maintain an ideal orientation to the Sun throughout the day. ${ }^{2}$ The effects of cloud cover and atmospheric disturbances could then be included in the value of the $I_{\operatorname{DirN}}$ by

$$
I_{\text {DirN }}=I_{\text {DirN }} \cdot(\text { CloudInput }+ \text { AtmVar })
$$

where AtmVar is the atmospheric disturbance present with or without cloud cover, and was approximated by setting it equal to a random number set to a normal distribution with a mean of .010 and a standard deviation of .0010. It varies each second, and represents the very slight fluctuations in irradiance seen even on relatively clear days. CloudInput is a measure used to describe the affect of clouds on the irradiance, and is explained later in this section.

Additionally, this increase in the diffuse irradiance due to a passing cloud can be seen before the decrease in direct irradiance due to the same cloud. This causes a slight rise in the overall output of the PV module just before a drop, and results from the way light that would otherwise have been directed to the side of the module is redirected to the module by the cloud just before it blocks the direct

\footnotetext{
${ }^{2}$ Here I have omitted reflected irradiance, which is the solar radiation incident upon a given surface after being reflected from a terrestrial surface such as the ground, ice, or water. It varies widely with any given application, but under most conditions constitutes a near negligible percentage of total irradiance, and is in fact considered negligible by most calculations in the photovoltaic industry.
} 
irradiance. Therefore, the larger and more opaque the incoming cloud, the higher the preliminary spike — and the larger the subsequent drop in output.

For the purposes of verifying the model's output to that of real-world data, it was necessary to convert the simulated data for global normal irradiance to global horizontal irradiance. Global horizontal irradiance $\left(I_{G H}\right)$ is the combined irradiance of direct solar energy and diffuse solar energy on a non-rotating horizontal surface (i.e. parallel to the ground throughout the day). This was the type of measurement taken by NREL's Oahu Solar Measurement Grid and can be derived from the direct normal and diffuse normal by the following equation:

$$
I_{G H}=\left(I_{D i r N}+I_{D i f f N}\right) \cdot \cos \left(\theta_{\odot}\right)
$$

The effects of the cloud cover, which are represented above by CloudInput, were not simple to formulate in an elegant way. There have been many approaches to this over the past few years, and it continues to be one of - if not the primary difficulties in planning for solar power generation. The difficulties of planning for the stochastic output of PV plants, which motivated the present study, are based primarily on the difficulties of cloud cover forecasting [38, 39, 40, 41].

The formulation I settled on in my model was a result of several iterated attempts to arrive at output results that sufficiently matched those from the Oahu facility, since it was not the goal of this thesis to find new methods of modeling cloud cover, but rather to find ways to implement current technology to overcome the stochasticity of the resulting PV power. First I would need to devise ways to compare the simulated solar irradiance data from the NetLogo model to data from the real-world in a way that would ensure that the data were reliable under a variety of conditions and starting scenarios. I discuss the metrics used to make this comparison in Section 2.2. The parameters used to configure the model to the 
specific site were from macro-level sources that could be applied to any region in the world.

\subsubsection{Photovoltaic Power Generation}

In regards to the photovoltaic array itself, there have been a few key parameters chosen to represent the vast majority of array types. There are many ways of categorizing photovoltaic arrays: by total area, by module material, as rotating/nonrotating, or as centralized/distributed, to name a few. The general type used as a reference in this model is that of a centralized utility-level array of reasonably high efficiency PV modules on a fixed horizontal mount. This type is one of the most frequently used in large array installations [42, 43].

Besides the fixed mount system — which is evident only in the fact that the simulated output is calculated as the global horizontal irradiance - other metrics include the efficiency of the PV modules, the desired peak power output, and the nominal voltage of the inverters on each string. These factors would in turn determine the overall size of the array and the number of modules per string. In general, the factors limiting the concentration of panels is simple compared to something like a wind farm, where myriad factors related to wind turbulence must be taken into account. With a PV array, the most important rule is that the arrays are spaced far enough apart to get the maximum uninterrupted irradiance from sunrise to sunset [44]. The closer they are together, the more of the twilight periods the inner modules will be shaded by the outer ones (at least in arrays with a grid-like formation). While the absolute difference in power generation from one minute to the next at these hours is minimal, one of the only limiting factors on the spread of PV modules is the size of the site being used for the farm, which is in turn usually limited by the property values in the area and the logistical constraints of building arrays above certain sizes. The general approach is that the modules are spaced just close enough together that an additional module would not fit between them. 
At that point it would obviously make more sense to install an additional module than to continue spreading the modules because of the nearly negligible increase in twilight-hour irradiance it would bring [44].

The electrical load on the system at any given time is another input to the system, and this varies on an hourly, daily, and seasonal basis. Because of the relatively small size of the average PV system, however, it is not expected that the output of any one facility will be sufficient to match the entire load of the power grid, and so instead the facility must simply meet agreed upon specifications for ramp rate and guaranteed overall power delivery.

\subsubsection{Battery Energy Storage System}

The battery energy storage system (BESS) that was integrated into the PV array in the model was based on a number of different industry projects that are either operational or under construction. The general setup of the BESS as it is designed in this model is that it would be connected to the grid at a common bus with a PV project. The initial design principles for this configuration used to implement the model are shown in Figure 2.2. There are other ways to integrate these energy storage systems into the grid, but this is one of the most common for its ease of control, and re-configurability. It is also common because it yields itself well to situations where utilities would want to construct a system at the substation for regional energy storage, as well as situations where a particular project manager would like to build a system at the substation to mitigate the variability of their privately held PV array nearby. The latter of these situations is the variety that much of this thesis is based on, and regardless of the owner of the PV array, many BESSs are setup based specifically on the parameters of a nearby solar site in terms of the anticipated required capacity and discharge capabilities. 


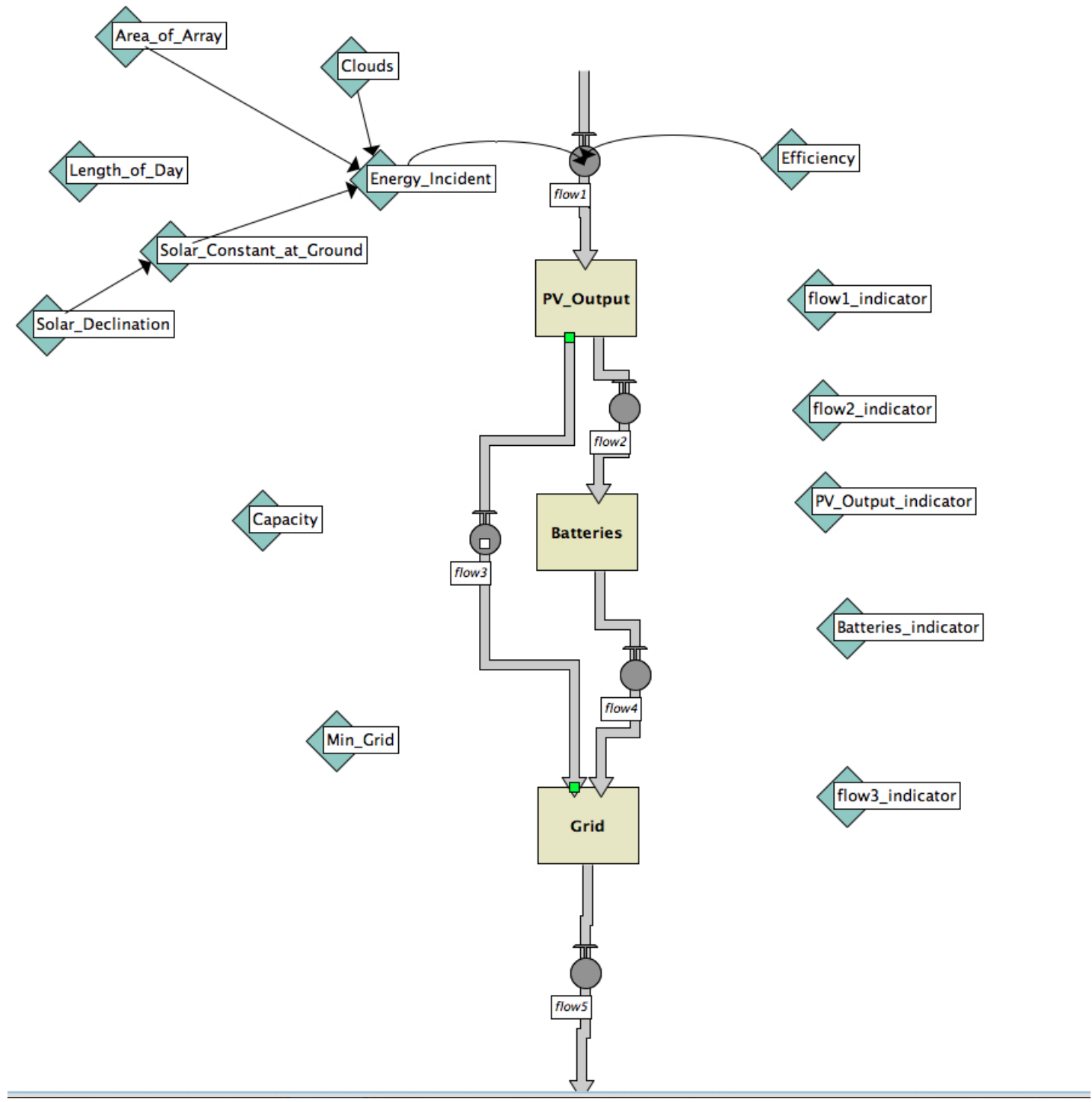

Figure 2.2: Preliminary design of the BESS model, showing the direction of causation between different user inputs, calculated variables, energy flows, and output measurements 


\section{Core Energy Storage Attributes}

The goal of this thesis has been to distill a variety of different technologies down to several core attributes, and to model these attributes in such a way that could be adjusted dynamically to observe the performance of the system under certain configurations. In order to observe the affect of the battery type on the performance of the system, the fundamental attributes of the battery type were determined to be

- peak charge/discharge rate

- energy capacity

- maximum depth of discharge

- capacity factor

- charge/discharge efficiency

\section{Battery Type}

The battery type is of the utmost importance in terms of the possible range of the parameters of the BESS. Some of the technical specifications of the battery types were not used explicitly in the model, but rather implicitly. The technical specifications for some battery types commonly used in grid-level storage are outlined in this section, as well as some less-commonly used ones - to give the reader a sense of perspective and why some qualities are preferred over others when choosing a battery configuration for a particular system. For each of these types, the relevant model parameter range is given and related to the chemical or physical makeup of the battery type. These are then summarized in Table 2.1.

Lead-Acid (Pb-Acid) batteries are known for their high power to weight ratio from high maximum current throughput, but are hampered by their low overall energy to weight ratios. It is for this reason that their primary application has been in traditional internal combustion automobiles as a method to supply power to the 
lighting and ignition systems [45]. They are not typically found in consumer electronics or electric vehicle applications because of this low energy to weight ratio. In applications where weight and size are unimportant, such as grid-level energy storage, they are more common. Still, they are often overshadowed in grid storage applications by other battery types such as NiCd and NiMH that offer substantially greater capabilities in terms of energy and power density, and average cycle life — albeit at a higher cost.

Nickel-cadmium (NiCd) batteries have similar applications to those of NiMH batteries. They have the disadvantage of being produced using the highly toxic metal cadmium. However until recently they were cheaper to produce than other similar batteries [45]. The lowering cost of production of $\mathrm{NiMH}$ batteries has led to stricter regulation on NiCd batteries for consumer use, though their use in specialized application remains popular because of their ability to tolerate high discharge rates with no loss of capacity or damage to the battery cells. They can also be discharged much deeper and for more prolonged periods of time than other batteries.

NiCd batteries have a charge cycle energy efficiency of between $60 \%$ and $90 \%$ - the upper extent of which places them ahead of almost every other battery type [45]. They have an energy density of $40-60 \mathrm{Wh} / \mathrm{kg}$, and a power density of $140-180 \mathrm{~W} / \mathrm{kg}$. The self discharge rate is low, at about $1 \%$ per day, though this would not likely be relevant in a BESS system connected at a common bus with a PV array [46]. In this setup, the battery would likely cycle once every 1-2 days. One of the more relevant aspects of NiCd batteries, however, is their cycle life which averages approximately 3000 charge cycles. This would be a major component of the lifetime cost of a BESS that selected this particular battery chemistry.

NiCd batteries are some of the most commonly used battery types in grid-scale 
energy storage. They are the battery type used in the world's current largest battery array - the Golden Valley Electric Association BESS in Fairbanks, Alaska. There, four strings of 344 battery modules connected in a series configuration to create a tested maximum output power of $46 \mathrm{MW}$ [47], though it is designed to deliver $27 \mathrm{MW}$ of power for a period of 15 minutes [48]. The project includes a total of 13,760 battery cells, and each battery has a stated anticipated life of between 20 and 30 years. The overall cost of the project is stated at $\$ 35$ million $[48]$.

Nickel-metal hydride (NiMH) batteries have two to three times the energy density of nickel-cadmium batteries - though less than that of lithium-ion. Their main disadvantage is a higher rate of self-discharge relative to other battery chemistries. They can discharge safely from $1.4 \mathrm{~V} /$ cell at full charge to a maximum discharge of just over $1 \mathrm{~V} /$ cell, with an average $1.25 \mathrm{~V} /$ cell during discharge, (under a current load of $0.5 \mathrm{~A}$ ). This delivers a more constant voltage over the entire charge cycle than other battery types, though over-discharging can damage the cells by polarity reversal $[49,50]$. NiMH batteries are well suited for high current drain applications because of their low internal resistance. They are often used in digital cameras, as well as electric automobiles.

NiMH batteries are well suited for many applications where high power-to-weight ratio is a priority. They are typically not chosen for grid-level storage because there is rarely a impetus to find a smaller, lighter battery - only one that stores enormous amounts of energy efficiently. Unfortunately, overall charge cycle efficiency is not a strong suit of a typical NiMH battery, with documented rates of between $50-80 \%$. Furthermore, their self-discharge rate without separator devices is considered relatively high. They have a working life of between 500 and 2000 cycles, depending on application [49]. Because of these reasons, they have not been included in the model. 
Molten salt batteries, (alternatively referred to as a liquid metal batteries or thermal batteries), are not reactive at ambient temperatures, but when heated to a certain threshold, they achieve a measure of power and energy density unmatched by many other batteries. Furthermore, because of the lack of necessity for thermal control devices required of most typical batteries, they are generally much cheaper to build and deploy. The three primary varieties of thermal batteries are sodiumion, sodium-sulfur, and magnesium-antimony [51].

Sodium-sulfur (NaS) batteries are a type of molten salt battery that consists of positive and negative electrodes of liquid sulfur and liquid sodium, respectively, separated from one another by a beta alumina ceramic electrolyte in a solid state [45]. While a phenomena referred to as thermal runaway is considered a potential problem that must be avoided in most batteries, part of the simplicity of $\mathrm{NaS}$ batteries and molten salt batteries in general is that their operating temperature is extremely high $\left(572-680^{\circ} \mathrm{F}\right.$ for NaS batteries), and what would be considered thermal runaway with other battery types is in fact the normal operating temperature for these types. In fact, heating is sometimes required when the battery is not in active use. Although the requirement of external heat requires additional energy, it is usually less than the cooling required of traditional battery types, and the systems required for this aspect of the battery bank are less complex. In most configurations, this heating is only required initially and during times of standby [52]. Finally, many of the required materials for the construction of these batteries is inexpensive and can be sourced locally — further decreasing the resultant cost and overall environmental footprint of any project utilizing these batteries.

The electrochemical specifications of NaS make them ideal for grid storage applications. They have an excellent stated cycle life that is dependent on the manner in which they are used. Their operating life is stated at 15 years, and the cy- 
cle is determined by the depth of discharge (DOD) at which they are operated. When operating at a DOD of $100 \%$ (i.e. the battery is discharged each charge cycle until no voltage difference remains), the cycle life will consist of 2500 cycles. When operating at a DOD of $85 \%$ (i.e. the battery is discharged each charge cycle until it arrives at a $15 \%$ charged state), the cycle life will consist of 4500 cycles [52]. Furthermore, their charge and discharge efficiency lies between $89 \%$ and $92 \%$, placing them well within the "high-efficiency" classification of batteries. These characteristics are greater than that in almost every other battery type.

While recent developments of molten salt batteries have been promising, such as those by Ambri and their magnesium antimony batteries [53], this battery type is still considered experimental, and real-world deployments of BESSs utilizing this battery chemistry are not yet common. However, one such example of this technology in action is the American Electric Power (AEP) Charleston Energy Storage project, in Charleston, VA. This project consists of a NaS battery array rated at 1.2 MW of instantaneous power, and an energy storage capacity of 7.2 MWh [52]. This is the first MW-scale NaS project outside of Japan, and it was installed to provide peak-shaving and grid support services at the local substation [54].

Lithium-ion (Li-ion) batteries have good energy efficiency rates of 85-95\%, and of course have excellent energy density and power density ratings of 100-200 Wh/kg and $360 \mathrm{~W} / \mathrm{kg}$, respectively. It is these characteristics that enable them to be the dominant form of energy storage in consumer electronics, where small size and weight are paramount. They have a relatively low self-discharge rate between $5 \%$ and $10 \%$ per month, depending on the specific type of Li-ion battery chemistry, and their working lifetime consists of approximately 3000 charge cycles — in line with previously mentioned battery types. Their cost is prohibitively high for most applications in grid-level energy storage, where larger, cheaper alternatives 
abound. The cost of lithium based batteries is expected to continue to rise as they are used more frequently in consumer electronics and electric vehicle applications, while the world's known supply of lithium is being depleted faster than new stores are discovered [55]. However, at the AES Laurel Mountain project in West Virginia, $32 \mathrm{MW}$ of Li-Ion batteries provide reserve capacity for a $97 \mathrm{MW}$ wind farm. The installation costs of the BESS are said to have been $\$ 900$ per $\mathrm{kW}$ [56], bringing the total installation cost to $\$ 28.8$ million.

Flow batteries are a type of electrochemical cell that functions in much the same way as a traditional battery, except for the fact that the electrolyte solution is not stored within the cell, but instead outside of the cell. This has several benefits in terms of scalability, as well as in the operational lifetime of the battery. It also offers benefits in terms of the pricing structure of the batteries, as a much higher proportion of the investment capital required for these batteries is in the form of on-going support and maintenance costs to maintain the requisite electrolyte solution levels, rather than in the initial and ongoing hardware operational costs. This is counter to most traditional battery technologies [57]. One of the most promising types of flow batteries, and the type included in this research, has been vanadium.

Vanadium flow batteries offer somewhat greater simplicity because of their use of only one electrolyte solution, rather than two as is the case with most other flow batteries. They can be discharged completely without damaging the system components, and their stated cycle life is greater than 10,000 cycles [57]. Their overall cycle efficiency, however, ranges from $65 \%$ to $72 \%$, depending on the manufacturer $(70 \%$ is the value used in the model). This contributes to an approximate overall lifetime cost of $\$ 915$ per $\mathrm{kWh}$. Vanadium flow batteries have a low energy-to-volume ratio, though this is less of a concern with grid-level energy storage applications. Additionally, though they constitute less complex systems than 


\begin{tabular}{|c|c|c|c|c|}
\hline Type & Min. Charge (\%) & Efficiency (\%) & Cycles & Cost $(\$ / \mathrm{kWh})$ \\
\hline Pb-Acid & 30 & 75 & 1500 & 135 \\
\hline $\mathrm{Ni}-\mathrm{Cd}$ & 0 & 75 & 3000 & 540 \\
\hline $\mathrm{Na}-\mathrm{S}$ & $0 / 15$ & 89 & $2500 / 4500$ & 500 \\
\hline Li-Ion & 20 & 93 & 3000 & 1145 \\
\hline Vanadium & 0 & 70 & 10000 & 915 \\
\hline
\end{tabular}

Table 2.1: Battery types included in the model, and their attributes, ordered approximately by their date of first demonstration

other flow batteries, they still require more complex systems than most other traditional batteries, and thus introduce more points of potential failure and required maintenance.

\section{BESS Component of Model}

In designing the BESS component of the model, I was determining the parameters that would most actively be manipulated to analyze the possible configurations of the energy storage at the PV site. In setting up the parameters, I looked at what values were available to project managers to change during the design phase of the system, (such as the charge and discharge speed, which are related to battery chemistry), as well as the parameters that would be adjusted from month-to-month, week-to-week or day-to-day by the facility operator or utility coordinator. These parameters included the application that was being utilized by the system, and according to which application was being used the parameters would include items such as the steady-state charge level, the output leveling target, or a parameter such as the point of transition from off-peak to on-peak power for time-shifting purposes.

The ramp rate was of course a primary constraint for each application, and when looking at what the ramp rate of the BESS should be, I looked at what the goal of a system operator would be in terms of how long the PV plant gave the utility to adjust other reserves when the plant came online in the morning or reduced 
its output in the evening. I felt that a good goal would be to reduce the need to utilize spinning reserves, if not the need to maintain reserves altogether. Spinning reserves are a source of wasted energy in that for a plant to be considered to have spinning reserves it must essentially "idle" with no power output so that if needed, it can ramp up in response to variabilities such as those from solar and wind power over the course of just a few minutes. The ability of any one plant to ramp up in response to variability is finite, with the best current natural gas plants only able to ramp up $63 \mathrm{MW}$ within a minute $[58,59]$.

We will not be able to completely eliminate the need for reserve capacity on the grid until we are able to store enough wind and solar energy to reliably power the grid 24/7. Therefore, non-spinning reserve will still be needed. Non-spinning reserve consists of power generation capabilities that sit in a ready state for when they are needed, but generally are not powered on continuously in the way that spinning reserves are. Non-spinning reserves generally require approximately 30 minutes to "spin-up" from their initial position, and so I felt that this 30 minute window would be a good goal for the model to achieve. Therefore, the ramp-rate constraint was defined to be not more than the nameplate power capacity of the PV facility over 30 minutes. Depending on the application, not all second-to-second variation that exceeded this would be eliminated. For example, in the Absolute Leveling application, the only ramp rates over the course of the day would be the initial ramp-up from zero to steady-state output, and the final ramp-down from the steady-state output back to zero. Both of these ramping periods would be constrained to last at least 30 minutes. However, for other applications, such as Reactive Leveling, some intermittent variability throughout the day that might be at a higher rate than 100\%/30 minutes would be allowed because of the caveat that variability within a certain threshold would be allowed, such as that within $10 \%$ of the peak output. 
In order to set both the ramp up and ramp down times to the minimum ramp period, the model had to first calculate the maximum projected output for that particular day. Therefore it first calculated the total instantaneous output of the system at culmination for that particular day, and then it set the maximum rate of increase or decrease to be that amount divided by 30 minutes. For the initial and final ramping periods, this was true down to the second-to-second timescales. For other ramping periods, the included timescales were dependent on the application.

\subsection{Model Testing}

In order to compare the simulated output from the model with observed data, several metrics were used to describe each dataset's variability. Using these, I would be able to objectively ascertain the differences between the two datasets. The metrics used included the following:

- Peak Global Horizontal Irradiance

- Average Global Horizontal Irradiance

- Total Daily Global Horizontal Irradiance

- Peak Variability

- Average Variability

\subsubsection{Comparison with NREL MIDC Data}

In order to verify that my model was working as intended, the results of the model needed to be verified against reputable real-world data. I would need data taken at the smallest timescale possible in order to observe the effects of battery storage on PV energy output, and for this, I turned to NREL's Measurement and Instrumentation Data Center (MIDC). Their data archives from the Oahu Solar Measurement Grid have some of the smallest timescales of any publicly available data, with second-to-second measurements of global horizontal irradiance for March 2010 through March 2011. A map of the Oahu site, with the solar modules marked accordingly, is given in Figure 2.3. 


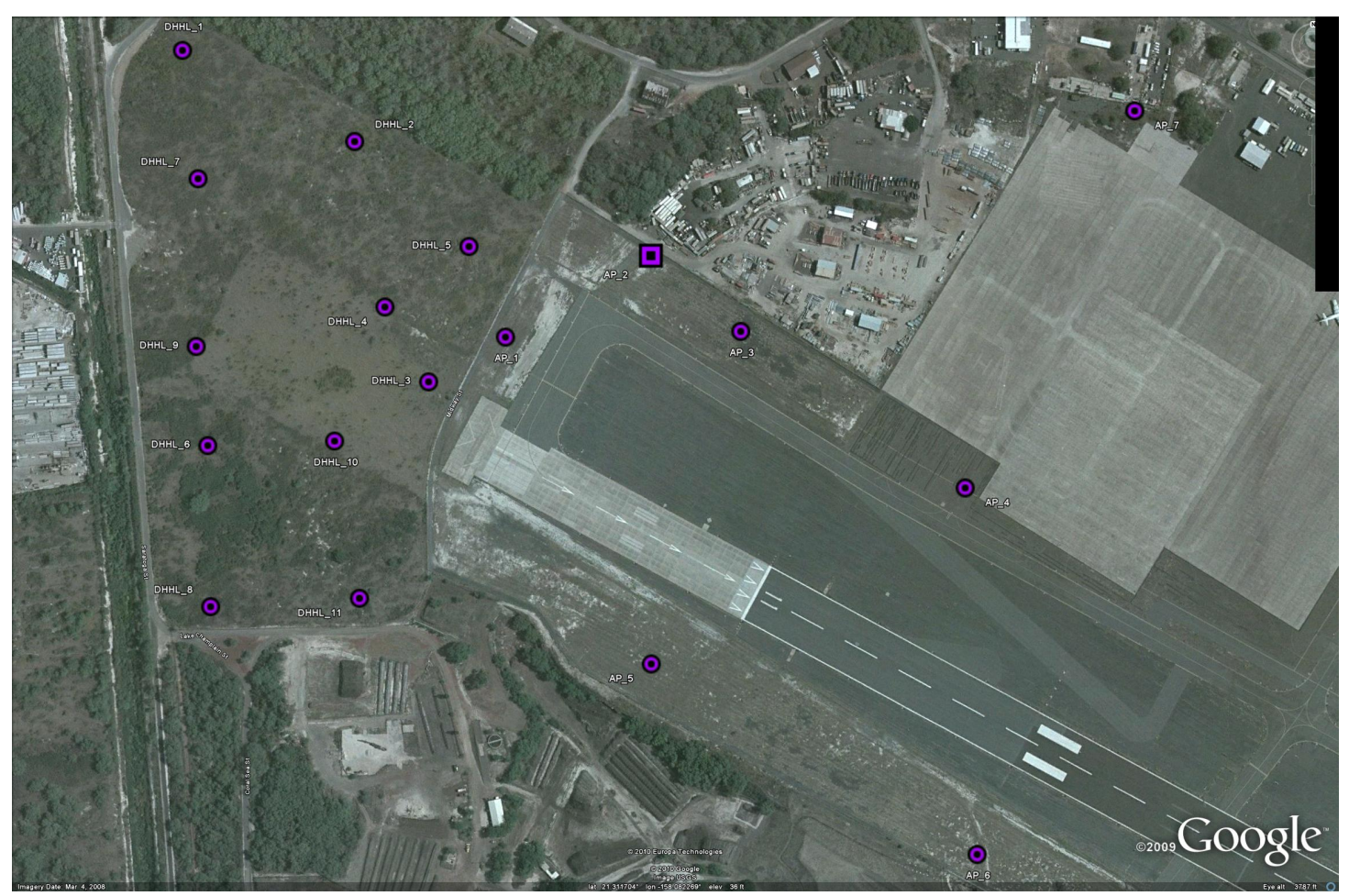

Figure 2.3: Map of the NREL Oahu site, showing the solar module placement

\section{Instrumentation at Oahu Site}

The second-to-second global horizontal irradiance data was collected across 17 LICOR LI-200 Pyranometer modules, which were mounted on 7 foot stationary tripods at an angle horizontal to the Earth's surface [60]. The error on these instruments has been observed at less than $5 \%$ under normal daylight conditions (i.e. when used to measure natural light, unobstructed by foliage) [61, 62, 63].

An important thing to remember about the data from Oahu is that, likely because of data storage constraints, NREL chose to only include data from 5:00am to $8: 00 \mathrm{pm}$. This is why there is not a full 86,400 data points per day, but instead 54,000. I also took out two of the columns of data (columns 13 and 15) for simplicity, because these were both global tilt measurements and would have confused the data.

Before making this comparison, the global normal irradiance simulated in the 
model was converted to global horizontal irradiance so that it would be the same type of measurement found in the NREL data. Converting my simulated data to the same type as that from NREL required the use of Equation 2.13.

$$
I_{D i r H}=I_{D i r N} * \cos \theta_{\odot}
$$

When comparing the datasets, I compared three different points, each a week apart, for four different months. Specifically, June 13, 20, 27; September 13, 20, 27; December 13, 20, 27; and March 13, 20, 27. Thus, I was able to find the systematic differences related to weather patterns from timescales of seconds up to several months (i.e. seasonal).

The average of the mean global horizontal for the dates used in September were only $0.07 \%$ less than the output of the model, and the average for the dates used in March were only 3.9\% higher. The higher variability in March $(-22.6 \% / 19.3 \%)$ relative to September $(-4.8 \% / 9.6 \%)$ was attributed to the greater amount of moisture in the air during March than September at this location. This aligned with documented climatic trends in this area, which showed an average precipitation level of $29 \%$ in March and 23\% in September [64]. The higher moisture levels would bring higher spikes of diffuse radiation - thereby raising the peak output, as well as more frequent drops in output due to outright cloud cover - thereby reducing the minimum output. The closeness of simulated output to the mean of these observed days indicated that there was not a systematic error evident at these times.

Conversely, an analysis of the simulated data for the months of June and December yielded a different picture altogether. The simulated data for the month of June was $18 \%$ higher than the average of the mean of observed data for that month. The extreme offsets of the observed data were also weighted rather low - at most, the simulated data was $41 \%$ higher than the observed data, and at a 
minimum it was only $.75 \%$ less than the observed data. This indicated that the simulated output for the month of June was approximately $18 \%$ too high. Furthermore, the simulated data for the month of December showed an even greater averaged error, though the variability in that month was much greater as well. The simulated data was $26 \%$ less than the mean of the averages across those dates in December, while the simulated data was at most $9.7 \%$ less than the averages of one of the dates, and at a minimum $32 \%$ less than the averages of one of the dates. ${ }^{3}$ Thus, this indicated that the simulated output for the month of December was approximately $26 \%$ too low.

Both of these results caused me to reevaluate the earlier stated approximation of the diffuse irradiance (which set it equal to $10 \%$ of direct irradiance across all dates and times). It would stand to reason that generalizing a measure of the sunlight reflected through the atmosphere would attribute too much diffuse irradiance to the summer months (when diffuse irradiance is lower because of less air mass from a less inclined solar arc, and less moisture in the air); and also attribute too little diffuse irradiance to the winter months (when diffuse irradiance is higher because of precisely the same reasons). I felt that tying the amount of diffuse irradiance to not only the direct irradiance, but also the solar declination would serve to better represent what would be observed in the real world.

\section{Improvements to Irradiance Model}

The adjustments that were made to the model at this point were based primarily on the findings of several papers outlining potential improvements to the calculation of direct and diffuse irradiance with varying amounts of cloud cover using empirical constraints [32, 36, 37, 65, 66]. For example, in Correlation Equation for Hourly Diffuse Radiation on a Horizontal Surface, the authors J. F. Orgill and K. G. T.

\footnotetext{
${ }^{3}$ This analysis omitted the values from two dates (December 20 and 27, 2010) that had outputs egregiously lower than the other four dates. This lower output was shown by the plots to be because of uncharacteristic cloud cover across those entire days, and thus these dates were not suitable for a comparative analysis of the solar output between different datasets.
} 
Hollands provide multiple fit equations for different clarity coefficients [66]. Using these results, the diffuse irradiance can be related to the overall irradiance with equation

$$
K=1.557-1.84 K_{T}
$$

for $0.35 \leq K_{T} \leq 0.75$, or days which are between $35 \%$ and $75 \%$ cloudy, whereas the relation

$$
K=1-0.249 K_{T}
$$

represents days that are less than $35 \%$ cloudy. The authors point out that, contrary to what one might think, the days which were very clear were the most difficult to predict. This is because on these days a cloud would often pass by without inhibiting the direct irradiance, but instead would act as a "diffuse concentrator" and reflect sunlight onto the modules from time to time. This would cause periodic and mostly unpredictable spikes in solar output — at times even exceeding the solar constant (which is the amount of solar radiation incident upon the Earth in the upper atmosphere, before being reduced by the process of passing through the gases and particulate matter that make up the atmosphere). Furthermore, there was relatively little data for days with this range of clarity, with the range $K_{T}>0.75$ representing only $5.6 \%$ of the dataset. For this reason, they specified a constant value of the $K=0.177$ for $K_{T}>0.75$, i.e., for days which were greater than $75 \%$ clear. Implementing these relations caused the model to align somewhat better with the observed data - especially on the types of particularly clear days mentioned previously.

\section{Comparison of Revised Model Output to NREL Oahu Data}

Figure 2.4 shows the peak and average output of the final model relative to the observed data from NREL's Oahu site. The observed data consists of the average for peak values across all the modules over the course of a day, and the average for average values across all the modules over the course of a day. I wrote a file IO 
script in $\mathrm{C}++$ to take each file (one for every day from 4/1/2010 to 3/31/2011), and find these metrics for that given day, and then process these into a file to be compiled as a year and then compared to the simulated data. This script is included in Appendix B.1.

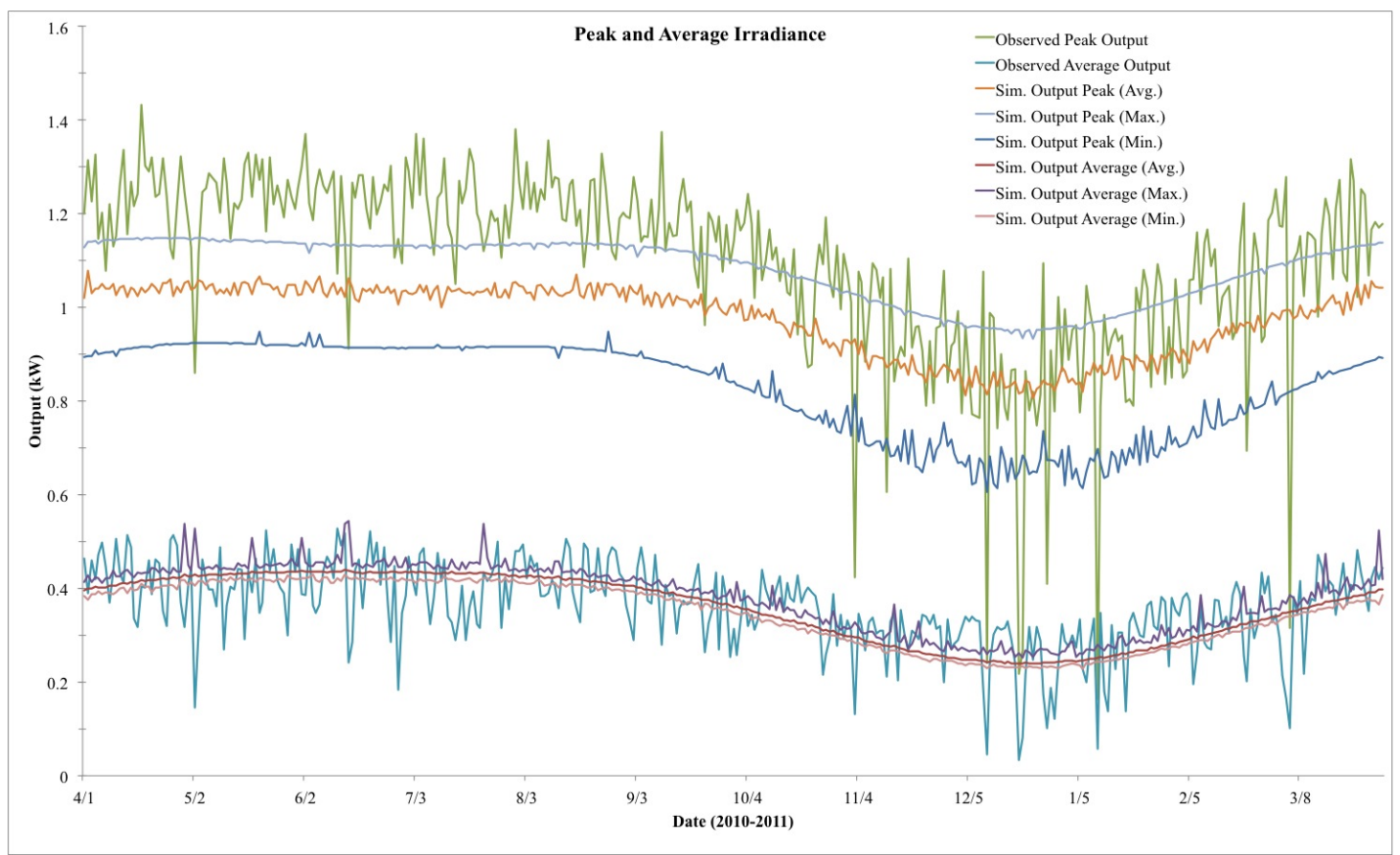

Figure 2.4: Comparison of both peak and average values for simulated and observed global horizontal irradiance data, using the NREL MIDC dataset

The simulated data consists of the mean average value of five model runs for each day of the year. Again, the plotted values reflect the average global horizontal and the peak global horizontal for the model as well. The model was configured with an array of default settings, which are manipulable in the model, and can be suited for any given geographical location. Several of the settings are fairly straightforward such as latitude, elevation, and the date to be simulated. Others, such as the various controls affecting cloud cover are representative of the most general terms concerning cloud cover, and possess the range to describe almost any conditions on Earth. They account for the fact that on a statistical level, cloud cover is one of the most mathematically random events that can occur in 


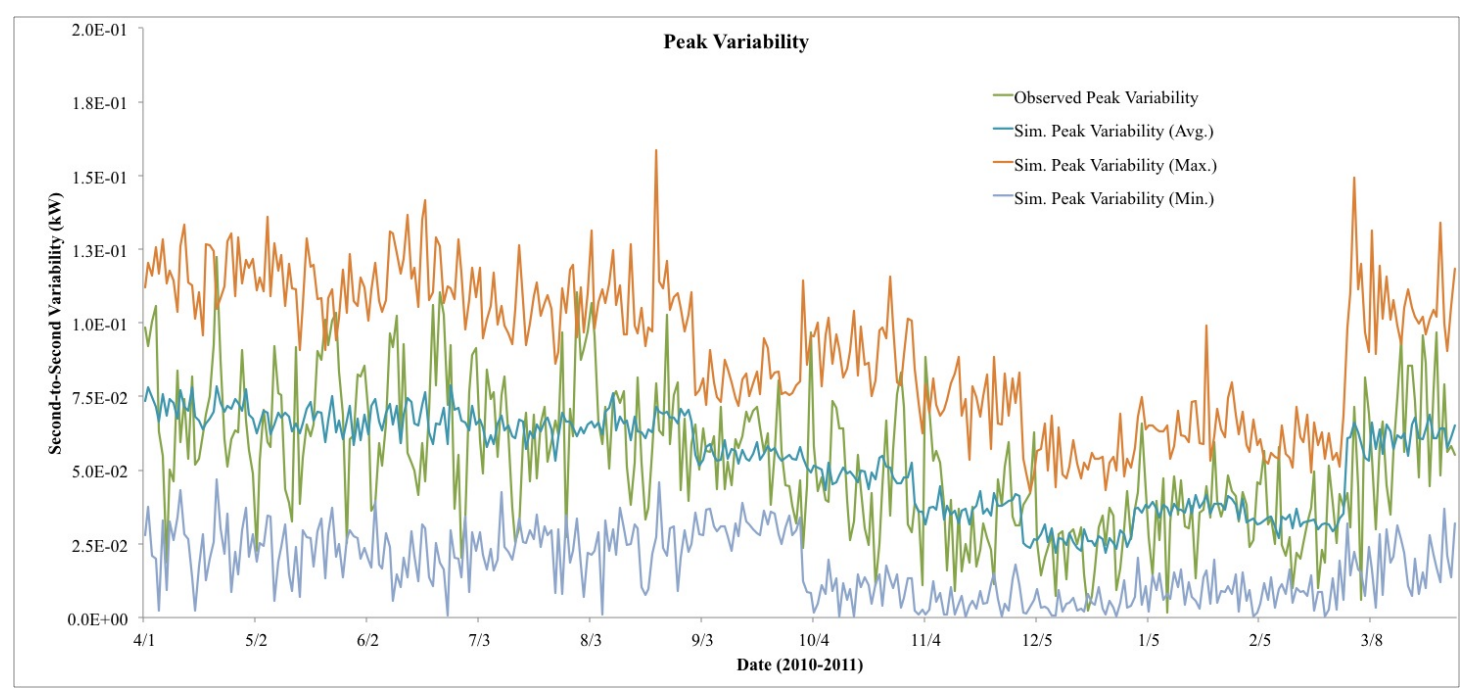

Figure 2.5: Comparison of daily peak second-to-second variability for simulated and observed global horizontal irradiance data, using the NREL MIDC dataset

nature, and modeling cloud cover for a given location with any level of accuracy is an entire field of science unto itself - and quite beyond the scope of this thesis. My goal was to give the user the ability to simulate the effects of this type of randomness with carefully constructed parameters. This creates the effect of cloud cover from a second-to-second to hour-to-hour timescale and matches reasonably well with the metrics for observed peak and average second-to-second variability in the data. These comparisons are shown in figures 2.5 and 2.6, respectively.

Overall, the data compared well. The daily peak and average irradiance outputs were $-11.25 \%$ and $-0.57 \%$ relative to the observed data, respectively. The daily peak and average variability measures were $0.41 \%$ and $12.88 \%$ relative to the observed data, respectively. These were calculated from the total amounts over the course of the year. Therefore the amount of irradiance simulated was somewhat lower than observed, while the amount of variability simulated was somewhat higher than observed. This was judged to be a reasonable estimation - because when calculating the feasibility of a potential BESS the effects of under-estimating output and over-estimating variability would serve to balance one another, and provide a realistic view of the possible improvements to be had from such a sys- 


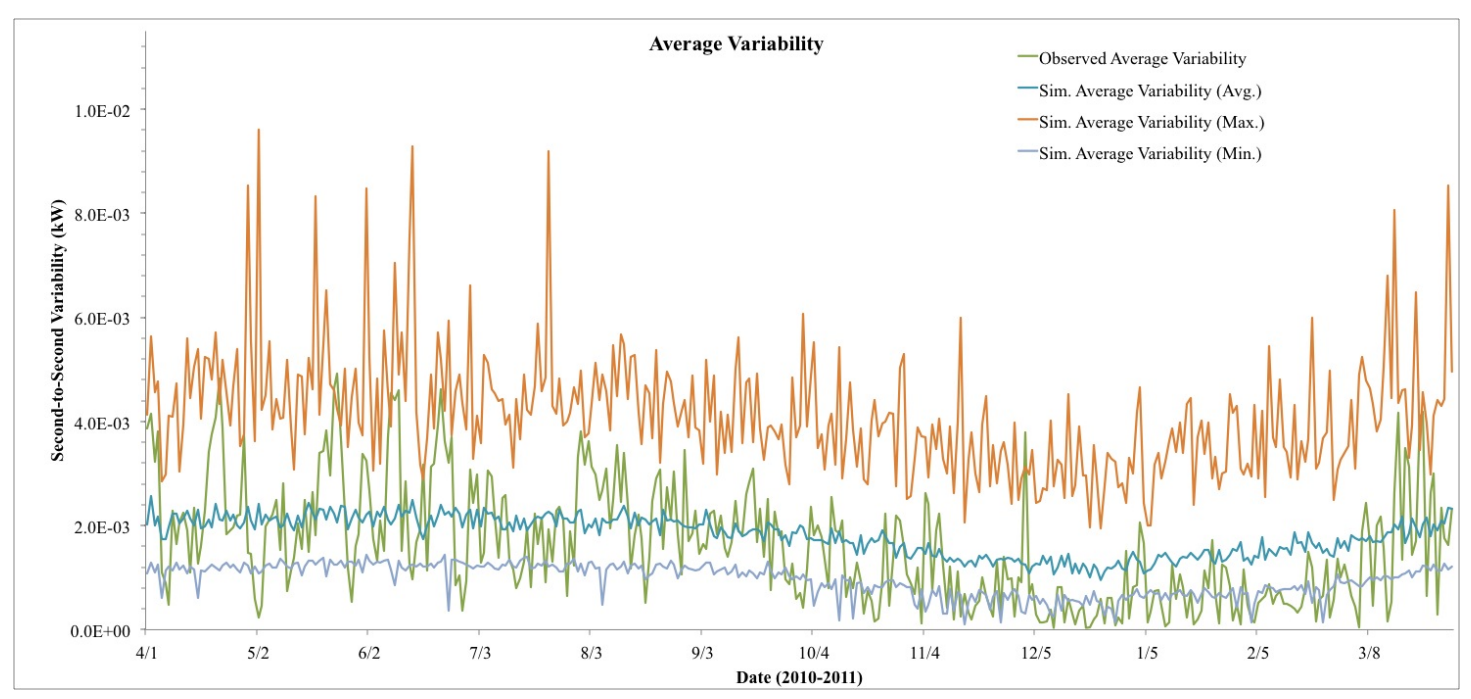

Figure 2.6: Comparison of daily average second-to-second variability for simulated and observed global horizontal irradiance data, using the NREL MIDC dataset

tem and, thus, whether it would prove a successful investment.

An important consideration, and something that is clear with a cursory glance of the global horizontal comparison data, is that while the model does a fairly good job of representing variability due to cloud cover throughout the day, it is ultimately highly deterministic in nature and thus the daily output changes very little from one day to the next. Large changes in output can only be seen hourly or seasonally. This is because, in the real world, some days will be extremely cloudy, and others will be quite sunny. For the purposes of ascertaining the mitigation of variability at timescales of several seconds, the model was purposefully built to give a mean value for the expected daily output. The cloud variables also remained unchanged throughout the year-long modeling experiment. Furthermore, this smoothing effect is made greater by the fact that the compared data given here was the result of averaging 30 different runs of the model with the same parameter settings for each individual day. 


\subsubsection{Comparison with UO SRML Data}

The initial ongoing comparison that was performed during the design stage of building the model was done with the MIDC Oahu second-to-second global horizontal irradiance data. This data was also then used to test the completed model, using the comparative metrics outlined previously. These datasets were shown to align well, yet because they were used both in the design and testing of the model, additional verification by other datasets was required to evaluate and minimize the effects of "over-fitting," whereby a model is tailored to a specific scenario - and does well in that scenario - but cannot be generalized for other variable inputs.

The University of Oregon Solar Radiation Monitoring Laboratory has archives of solar data from monitoring stations across the Pacific Northwest. These datasets consist of varying measurement types, data periods, and sample rates. One of their datasets with the highest granularity (i.e. sample rate) is collected at their Eugene, Oregon station. Data is collected here at one minute time intervals by a YES Multifilter Rotating Shadowband Radiometer, and recorded using a YESDAS-2 datalogger. It collects measurements continuously, calculating the diffuse irradiance when the shadowband is directly over the sensor and the global irradiance when the shadowband is at rest. It is then able to calculate the direct irradiance from these values. The unit is mounted on a stationary platform pointed directly up. It therefore captures only the horizontal component of irradiance [67].

Figure 2.7 shows that when comparing the simulated and observed irradiance, the peak of the curve, as well as its shape, are fairly identical over the course of an average day. The slight offset to the left is symptomatic of the approximation of solar noon in the model, as well as a slight underestimation of the diffuse irradiance from cloud cover, which affects the results most in the morning and evening, and least at midday. The data in Figure 2.8 shows significant differences 


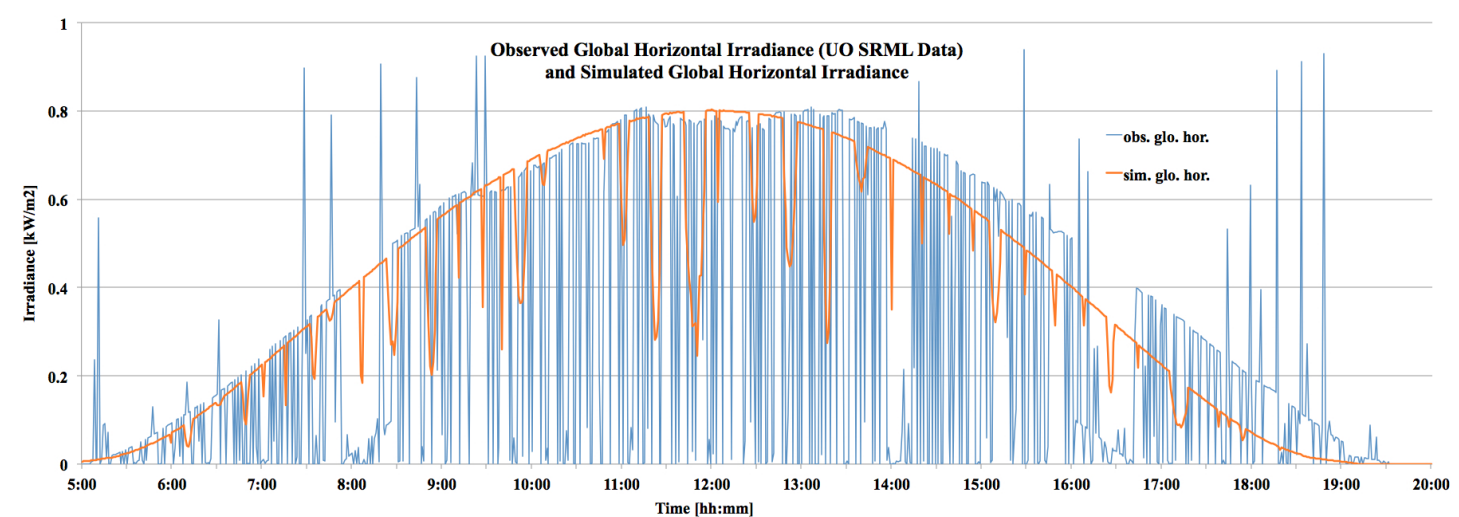

Figure 2.7: Comparison of simulated global horizontal irradiance data with data observed at Eugene, Oregon solar monitoring site by the University of Oregon SRML on August 2, 2010. The model was set to the same date, latitude, and elevation as the monitoring site.

between the simulated and the observed data over the course of the year. In the observed data, the highly opaque and seasonally intermittent cloud cover of the Willamette River Valley caused the second-to-second variability of the observed horizontal irradiance to be much higher than in Oahu; thus the average output was much lower than expected. This difference in average second-to-second variability between the simulated and observed data for the Oregon site is shown in Figure 2.9. While the model can be tailored to describe cloud cover more accurately by adjusting the cloud cover parameters to reflect local weather patterns, doing so for multiple sites was outside the scope of this thesis.

\subsubsection{Model Parameters}

For the research into which configuration of a potential BESS would be the most feasible, I set up the model to simulate the output of such a system by varying a number of parameters. Each of these parameters governed a particular component of the system, some of which approximate environment conditions, while others describe system design and configuration variables. A sensitivity table of these parameters is given in Table 2.2. The parameters of the model that describe environmental factors local to a particular site are shown with the ${ }^{l o c}$ qualifier, 


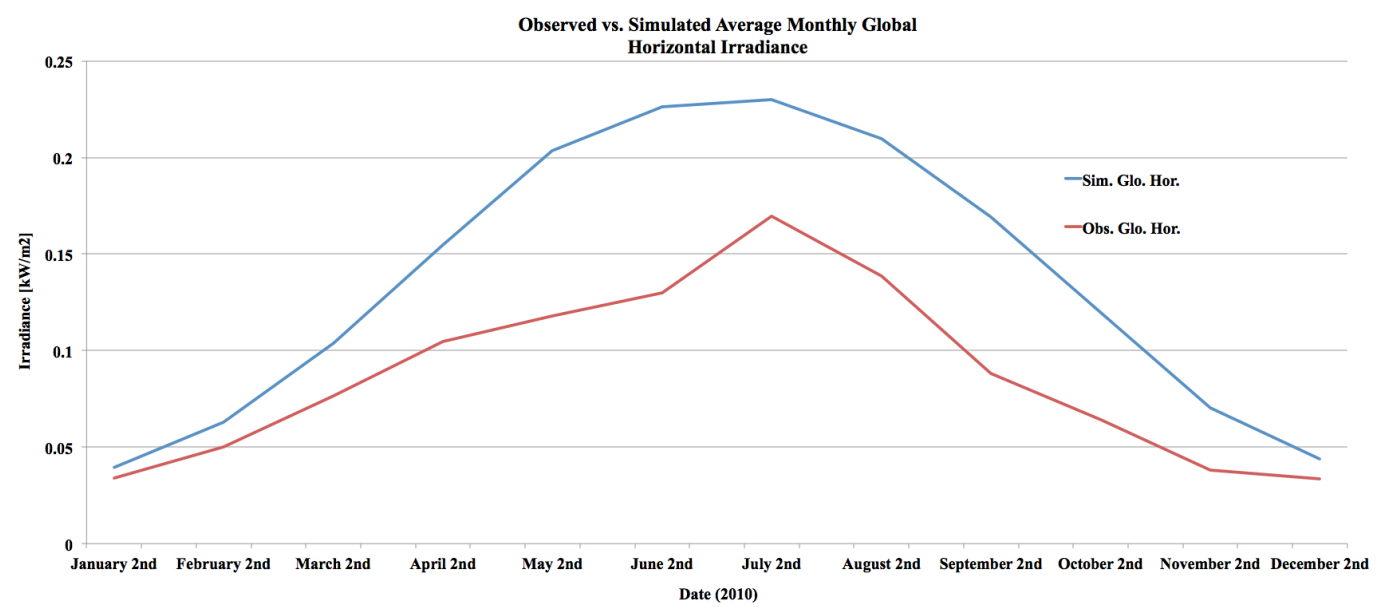

Figure 2.8: Comparison of monthly simulated global horizontal irradiance data with data observed at Eugene, Oregon solar monitoring site by the University of Oregon SRML on August 2, 2010. The model was set to the same latitude, and elevation as the monitoring site. The overestimation of the model's output in this case was seen to be related to the underestimation of the average variability at this site.

and the default values for these are given as the values for the NREL Oahu site. The parity among the standard deviation of the $\mathrm{R} \_$Ad changes is reflective of the fact that much of the variance in the result of changing a particular parameter is caused by variances in the stochasticity of the system that occur even when all parameters remain unchanged.

Many aspects of the model are shown here, but one seemingly peculiar finding warrants some explanation. The Site_Elevation parameter is shown here as having zero effect on the $R_{A d}$ value, on average. However this is an effect of the fact that the Oahu site is nearly at sea level (an elevation of approximately $10 \mathrm{~m}$ ). Therefore a change in $20 \%$ of this value is only a change of $2 \mathrm{~m}$ in either direction, and this has a nearly negligible effect on the atmospheric absorption coefficient, which represents the amount of atmosphere that sunlight must travel through to reach the ground. Conversely, if the starting value of the Site_Elevation parameter was the elevation of the highest point on the island of Oahu $(1.22 \mathrm{~km}$, or approximately 4000 feet) then changing the elevation by $20 \%$ would result in a change of $244 \mathrm{~m}$. 


\begin{tabular}{|c|c|c|c|c|}
\hline Parameter & $\begin{array}{r}\text { Parameter } \\
\text { Default }\end{array}$ & $\begin{array}{l}\text { Parameter } \\
\text { Change }[\%]\end{array}$ & $\begin{array}{r}R_{\text {Ad }} \text { Change }[\%] \\
\quad \text { (avg. } 30 \text { runs) }\end{array}$ & $\begin{array}{c}\text { St. Dev. of } \\
R_{A d} \text { Change }\end{array}$ \\
\hline ALL DEFAULT & $\mathrm{N} / \mathrm{A}$ & $-20 / 20$ & $0.00 / 0.00$ & $68 / 68$ \\
\hline BESS_Capacity_pct & 0.5 & $-20 / 20$ & $-4.9 /-11$ & $60 / 69$ \\
\hline BESS_SS_Charge & 0.5 & $-20 / 20$ & $-7.7 /-1.1$ & $67 / 63$ \\
\hline Leveling_Target_pct & 0.5 & $-20 / 20$ & $-5.2 /-29$ & $43 / 84$ \\
\hline atm_var_avgloc & 0.010 & $-20 / 20$ & $0.67 /-7.6$ & $61 / 70$ \\
\hline cloud-size_avg $^{l o c}$ & 1000 & $-20 / 20$ & $-16 /-3.2$ & $65 / 58$ \\
\hline cloud_opacity_min ${ }^{l o c}$ & 10 & $-20 / 20$ & $-3.0 / 0.90$ & $66 / 70$ \\
\hline cloud_opacity_max ${ }^{l o c}$ & 80 & $-20 / 20$ & $5.0 / 2.0$ & $45 / 63$ \\
\hline cloud-distance_avgloc & 15000 & $-20 / 20$ & $9.6 /-23$ & $67 / 65$ \\
\hline cloud-speed_avgloc & 7.0 & $-20 / 20$ & $15 / 6.5$ & $58 / 58$ \\
\hline Latitude $^{l o c}$ & 21.31276 & $-20 / 20$ & $-12 / 4.3$ & $65 / 70$ \\
\hline Site_Elevation $^{l o c}$ & 0.0010 & $-20 / 20$ & $0.00 / 0.00$ & $68 / 68$ \\
\hline PV_Efficiency & 0.145 & $-20 / 20$ & $-32 / 17$ & $55 / 75$ \\
\hline V_Nom_DC_of_Inverter & 1000 & $-20 / 20$ & $-0.035 / 1.7$ & $68 / 66$ \\
\hline Desired_Parray_max_kW & 5700 & $-20 / 20$ & $-18 / 12$ & $47 / 79$ \\
\hline Time-to-Start-Sim_h & 5 & $-20 / 20$ & $6.9 /-1.6$ & $57 / 62$ \\
\hline Time-to-End-Sim_h & 20 & $-20 / 20$ & $-16 /-0.78$ & $61 / 68$ \\
\hline BESS_Min_Charge & 0.13 & $-20 / 20$ & $0.62 /-1.7$ & $67 / 68$ \\
\hline BESS_Efficiency & 0.85 & $-20 / 20$ & $-3.3 / 1.0$ & $68 / 66$ \\
\hline Cycle_life & 4000 & $-20 / 20$ & $-24 / 16$ & $68 / 68$ \\
\hline C_BESS & 620 & $-20 / 20$ & $19 /-19$ & $68 / 68$ \\
\hline V_at_Pmodule_max & 33.5 & $-20 / 20$ & $-0.31 / 0.78$ & $66 / 66$ \\
\hline Pmodule_max_kW & 0.285 & $-20 / 20$ & $30 /-25$ & $57 / 49$ \\
\hline P_Re $e^{l o c}$ & 0.1031 & $-20 / 20$ & $-93 / 93$ & $35 / 100$ \\
\hline P_Ex ${ }^{l o c}$ & 0.06 & $-20 / 20$ & $54 /-54$ & $87 / 49$ \\
\hline Discharge_Time & 9 & $-20 / 20$ & $-8.8 / 0.00$ & $68 / 68$ \\
\hline rr_limit & 3 & $-20 / 20$ & $-0.02 / 0.02$ & $68 / 68$ \\
\hline module_area & 1.752192 & $-20 / 20$ & $-27 / 10$ & $47 / 75$ \\
\hline gcr & 0.20 & $-20 / 20$ & $5.3 /-0.00506$ & $55 / 60$ \\
\hline Month_initial & 6 & $-20 / 20$ & $-88 /-88$ & $30 / 30$ \\
\hline Day-of-Month_initial & 15 & $-20 / 20$ & $-1.05 /-1.8$ & $68 / 67$ \\
\hline
\end{tabular}

Table 2.2: Sensitivity table for the parameters used in the model. Each parameter was systematically decreased by $20 \%$ and then increased by $20 \%$, without any other changes in the model setup, to see how changes in each parameter affected the additional revenue $\left(R_{A d}\right)$ metric that would later be used to compare the different configurations; local parameters are denoted by the ${ }^{l o c}$ qualifier, and have their defaults set to that of the NREL Oahu site 


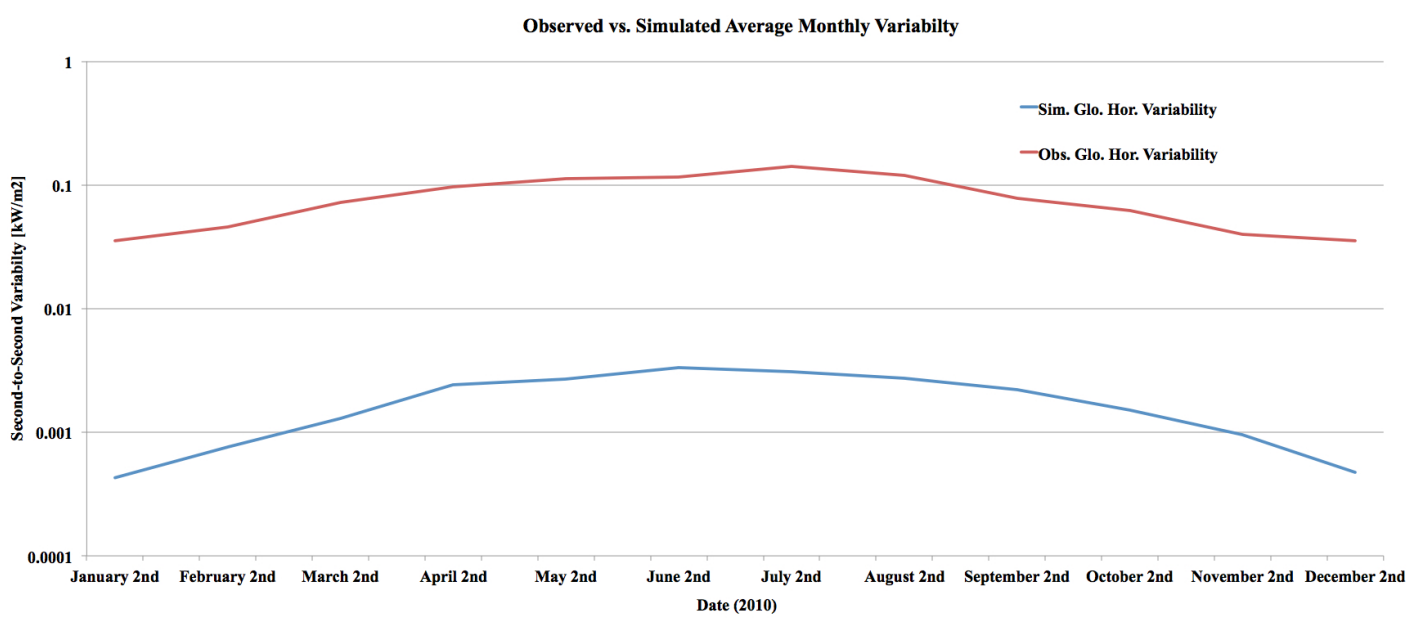

Figure 2.9: Comparison of simulated and observed average second-to-second variability, averaged over each month, using the University of Oregon SRML dataset (logarithmic scale)

Increasing or decreasing the elevation by this amount would change the resulting $R_{A d}$ by $1.2 \%$, or $-1.5 \%$, respectively.

\subsection{Search for Reasonable Configuration of Battery Parameters}

After properly implementing the model and designing the different relevant parameters, the direction of the research was to find how adjusting these changed the output of the model, primarily in terms of average and overall variability, and thus the improvement in ramp rates to be expected. Improving the average ramp rate is typically the overall purpose behind grid-level energy storage systems that are in use today. While ancillary services such as frequency response are not the driving need behind such systems, they do often provide additional incentive or complete the overall financial profile of the project to the point of long-term viability $[68,69]$. Therefore, they are important, yet were not included in the model outlined in this thesis because of the significant additional required computational time for measures as continuously variable as voltage frequency, and the benefits of including a survey of this capability did not outweigh the incurred drawbacks. 
Within the scope of power response there are several different applications that could be selected for the model, which represent different priorities a utility or project manager might have at a given time. A reasonable configuration of the parameters of the model are, therefore, specific to each application. The ideal combination of energy capacity and peak power capability for the purpose of ramp rate regulation will be different from the ideal combination of these for the purpose of peak-shifting. This type of system design is parallel to that seen in current projects such as the Golden Valley Electric Association BESS in Alaska, which is designed with 7 different "modes of operation" — mostly pertaining to improving the reliability of the overall power generation at the local substation, but also including modes of operation pertaining to frequency support and other auxiliary services to the grid [47]. The applications chosen for the NetLogo model outlined in this thesis included the following:

- Absolute Leveling

- Reactive Leveling

- Time-Shifting

\subsubsection{Absolute Leveling}

The Absolute Leveling application represented a scenario whereby the utility or project manager would want a constant output over a certain period of time. In this case, the peak output over that time would take less precedence than the lack of any variability whatsoever - so the resultant output to the grid would be less during the middle of the day than the raw PV output, but would be greater than the raw PV output earlier in the morning and later in the afternoon. This application is somewhat less common in practice at the current time than reactive leveling because of the considerable energy storage capacity requirements.

The search for reasonable configurations for this application was set up to maximize the additional revenue generated by a BESS by minimizing the variability 
of the system (by maximizing the period of time covered by the leveled output), while reducing the output power by a minimal amount. Furthermore, the search factored in the costs associated with different battery types to provide a more comprehensive view of the system's feasibility than an outline of the capabilities of the system could provide. This application constituted the majority of the research of this thesis because of its ability to be determined by relatively few metrics allowing for greater granularity in the parameter space covered in the experiments outlined in Section 3.1 .

\subsubsection{Reactive Leveling}

Reactive leveling is one of the more common applications of a typical BESS in the marketplace today, because they typically balance the contradicting needs of consistent output with relatively low battery costs. This is possible because, instead of mandating that a constant output be maintained over a period of time, the utility or project manager could simply require that the worst variations in output be mitigated. Therefore, instead of requiring that hours of energy be stored, only 15-30 minutes worth of peak power capacity are required to mitigate the very large drops in output that can occur suddenly with PV power, but typically do not persist for very long. In the case of sudden large drops that begin to persist longer than expected, the BESS can be programmed to begin to ramp down over a period of time to give utility planners adequate time to ramp up other resources. In this sense they address the concerns over drastic variability in PV to allow them to be more readily implemented, while not demanding that their variability drop to zero - which allows the costs of the BESS portions of the project to remain manageable.

The search for a reasonable configuration for this application would be set up to maximize the output over the course of the day by varying the maximum allowed variability, the steady-state charge, and a number of other charge variables. 
The constraints of the problem would be related to the need for a certain output level and a certain maximum energy storage capacity.

\subsubsection{Time-Shifting}

The third application of the model was that of Time-Shifting. This represented the times when there is a sufficiently large disparity between peak and off-peak power costs to incentivize storing off-peak power to be discharged during peak times. The general logic governing this application is that the BESS will store as much energy as possible during off-peak hours - even if this means allowing the output of the facility to fluctuate with the changing solar energy while it is reaching its constant charge state - and then discharging this power when the price is forecast to be the highest. This application in its pure form is also relatively rare in a typical BESS at this point in time because generally the cost of storing the energy in terms of the required battery units is greater than the potential profit from selling the stored energy at peak prices. This application was included in the model to address the theoretical potential capabilities of a BESS under certain conditions.

The search for a reasonable configuration for this application would be set up to maximize the amount of energy that could be stored for a given amount of time by adjusting the charge parameters. The constraints would include those related to maximum energy storage capacity due to battery costs, as well as feasibility measures related to battery chemistry and cell breakdown due to long-term energy storage at a constant capacity. 


\section{Results}

\subsection{BehaviorSpace Experiments}

BehaviorSpace is a tool included with NetLogo that allows users to set up extensive and wide-ranging experiments with their models to discover their behavior under a variety of conditions. Essentially, it automates the process of varying the parameters of a model and records the results of each run with these different parameters. This is what the NetLogo documentation refers to as "parameter sweeping" [70], though in practice it is similar to the so-called "brute force" methods used in other simulation environments. The purpose and strength of the tool lies in taking samples from a broad parameter space to look for trends toward a particular behavior of interest. It can certainly do this more quickly and reliably than a human operator could manually change the settings and record the results of each run.

However, because of the small number of samples, the BehaviorSpace tool is relatively less comprehensive than other true optimization tools. This is especially true in non-linear problems such as those often found in science and in fields with multiple variables that are not truly independent but instead interact in complex ways. In these cases, BehaviorSpace will often point to a local minima or maxima instead of a global minima or maxima meaning that any optimized solution must be taken as the optimized solution of the sampled set, and not of the problem as a whole. For this reason, the results given by BehaviorSpace will certainly be useful as an indication of the general trends and relationships of the system, but are not meant to represent a mathematically rigid optimization of the system. 
The experiments prior to Experiment 9 served mainly as evaluations of the comparative capabilities of various BESS configurations to simply mitigate the variability in PV output, regardless of their incurred cost. Therefore they should be seen as an indication of the future potential for the respective battery technologies, and the relationship between different design parameters, but not necessarily what is feasible at present. These initial experiments also served to aid in the evolutionary design of the model. The results of Experiments 9 through 11, represent the final stage of the model design and are the most realistic representation of what could be expected from a potential BESS.

\subsubsection{Experiment 1}

The first BehaviorSpace experiment I ran consisted of varying the Steady State Charge (BESS_SS_Charge), the Minimum Charge (BESS_Min_Charge), and the Leveling Target (Leveling_Target) for the output over four days each over four months. The text input for the experiment is shown below:

["BESS_SS_Charge" [0.2 0.21$]$ 1

["BESS_Min_Charge" [0.05 0.05 0.2] $]$

["Leveling_Target" [3000 400 5800]]

["Month_initial" 3609 12]

The general syntax of this follows the form ["parameter" [initial_value increment_size final_value]]. Alternatively, for sufficiently few variations, the form ["parameter" variation_1 variation_2 variation_3 ... ] was used. This created an experiment of 640 runs, each corresponding to a unique combination of the parameters. These results are shown in Table 3.1.

The results of this experiment showed a minimum average variation of $0.020 \mathrm{~kW} / \mathrm{s}$ (measured in terms of second-to-second variation of overall system output in $\mathrm{kW}$ ). This corresponded to a BESS_SS_Charge of .2, a BESS_Min_Charge of .05, and a Leveling-Target of 3400, during the month of March. The model output with this 


\begin{tabular}{lrrr}
\hline & lowest: & 2nd lowest: & 3rd lowest: \\
\hline Avg_Sys_Var & 0.020 & 0.029 & 0.039 \\
Run\# & 5 & 329 & 139 \\
BESS_SS_Charge & 0.2 & 0.6 & 0.4 \\
BESS_Min_Charge & 0.05 & 0.15 & 0.05 \\
Leveling_Target & 3400 & 3800 & 3800 \\
Month_Initial & 3 & 3 & 9 \\
Peak_Sys_Output & 3400 & 3800 & 3800 \\
Avg_Sys_Output & 1900 & 1900 & 2100 \\
\hline
\end{tabular}

Table 3.1: Lowest variability configurations from BehaviorSpace Experiment 1

configuration is included in Figure 3.1.

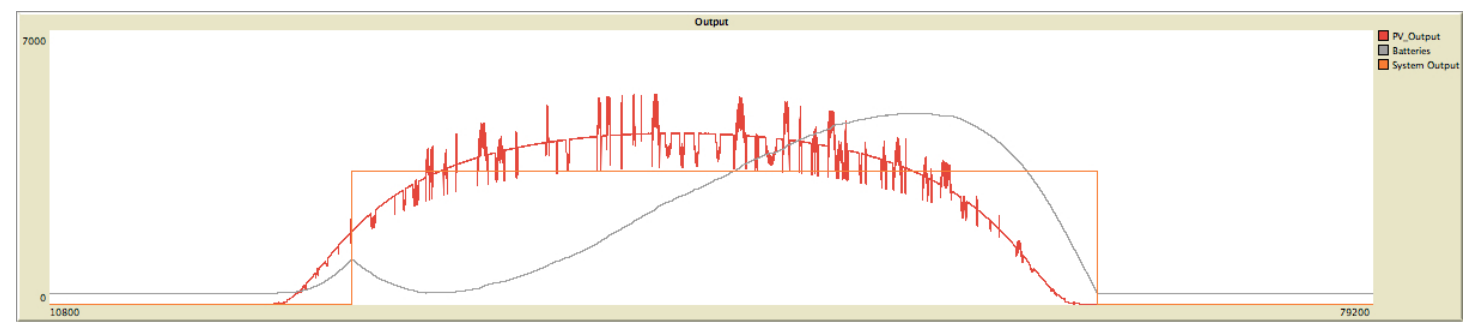

Figure 3.1: Output of an optimal configuration of BehaviorSpace Experiment 1. The values for PV Output and System Output are in units of kW, while the plot for Batteries is in units of $\mathrm{kWh}$, and represents the charge state of the BESS.

While likely not a true global optimum, this output was a relatively accurate illustration of the overall behavior that would be expected from the Absolute Leveling application - in that while the peak system output for the day was only $63 \%$ of what the PV array produced at its peak $(3400 \mathrm{~kW}$ System Output from a PV Output of approximately $5400 \mathrm{~kW}$ at noon), the average output was exactly the same, at $1900 \mathrm{~kW}$, respectively. The difference in average variability, however, was stark: the average variability of the system's output when utilizing the BESS was $.08 \%$ of the average variability when not utilizing the BESS (an average of 0.02 $\mathrm{kW} / \mathrm{s}$ with the BESS versus $0.24 \mathrm{~kW} / \mathrm{s}$ without). 


\subsubsection{Experiment 2}

Since the first experiment emphasized the need for increased granularity in the parameters, the second BehaviorSpace experiment I designed excluded the month of the year as an independent variable. For Experiment 2, I set up the parameter space as follows:

["BESS_SS_Charge" [0.05 0.051$]$ 1]

["BESS_Min_Charge" [ $\left[\begin{array}{lll}0.025 & 0.025 & 0.2\end{array}\right]$

["Leveling_Target" [3000 50 5800] ]

This brought the number of runs required to 9120, and with increased granularity came drastically increased computing time. The required time for the first experiment of 640 runs was about 25 minutes, and with this experiment the simulation time increased linearly to over 6 hours for 9120 runs. This also indicated a drawback of the parameter sweeping method - the tradeoff in computing time for even modest increases in sample granularity were relatively large.

Still, the results were intriguing. It showed a minimum simulated average variability of $0.013 \mathrm{~kW} / \mathrm{s}$. The parameter configuration that gave this consisted of a BESS_SS_Charge of .3, a BESS_Min_Charge of .075, and a Leveling_Target of 3400. This experiment, as the first one, was run with a constant overall BESS_Capacity of $5800 \mathrm{kWh}$. The parameter configurations with the five lowest average variability are shown in Table 3.2 .

One of the interesting things that was made evident by the results of this experiment was that although the minimum allowable charge was .05, the optimal Min_Charge in four of the five lowest variability configurations was .075. The output from the configuration of lowest average variability is shown here in Figure 3.2. Here, the SS_Charge is set to .3, the Min_Charge is set to .075, and the Leveling_Target is set to 3400 . 


\begin{tabular}{lrrrrr}
\hline & lowest & 2nd lowest: & 3rd lowest: & 4th lowest: & 5th lowest: \\
\hline Avg_Sys_Var & 0.013 & 0.015 & 0.017 & 0.019 & 0.021 \\
Run Number & 2403 & 2861 & 3778 & 3376 & 1946 \\
BESS_SS_Charge & 0.3 & 0.35 & 0.45 & 0.4 & 0.25 \\
BESS_Min_Charge & 0.075 & 0.075 & 0.075 & 0.1 & 0.075 \\
Leveling_Target & 3400 & 3500 & 3750 & 3600 & 3350 \\
Peak_Sys_Output & 3400 & 3500 & 3800 & 3600 & 3400 \\
Avg_Sys_Output & 1900 & 1900 & 1900 & 1900 & 1900 \\
\hline
\end{tabular}

Table 3.2: Lowest variability configurations from BehaviorSpace Experiment 2

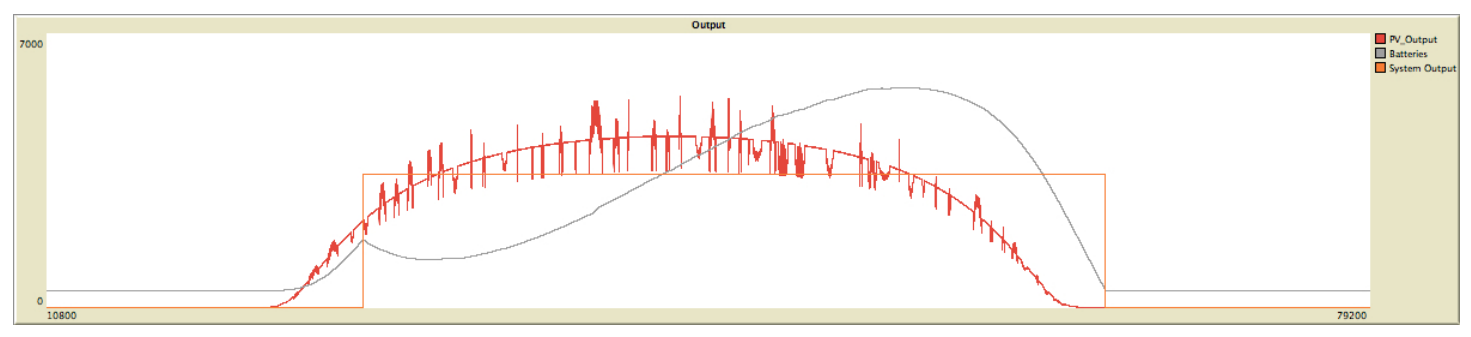

Figure 3.2: Output of an optimal configuration from BehaviorSpace Experiment 2. The values for $P V$ Output and System Output are in units of $\mathrm{kW}$, while the plot for Batteries is in units of $\mathrm{kWh}$, and represents the charge state of the BESS.

\subsubsection{Experiment 3}

The scenario represented in Experiment 3 is one where the PV array has been built, and a certain level of output is desired at a continuous rate during the entire operational period. The fundamental design choices would be what type and capacity of battery storage to install. The type of battery would cycle through the model and change the Min_Charge accordingly. ${ }^{1}$

To improve the efficiency of this experiment, a UI-facing variable called "Battery_Type" was added. This would enable a number of the parameters related to the type of battery chosen for a project to be set in the back-end code. Thus, the model would take on the following structure:

\footnotetext{
${ }^{1}$ For the NaS battery type, Min_Charge levels of both $0 \%$ and $15 \%$ were simulated, with the secondary effect of changing the cycle life between 2500 and 4500 cycles, respectively.
} 
- Model parameters to be varied directly by BehaviorSpace:

- BESS_Capacity

- BESS_SS_Charge

- Leveling_Target

- Discharge Time

- Battery_Type

- Model parameters to be set by varying battery type:

- BESS_Min_Charge

- BESS_Efficiency

- Cycle_Life

- Cost_BESS (per kWh Capacity)

The optimum configuration would then be found by, first, finding the ratio of cumulative variability of the output with the BESS system and without it, hereafter referred to as the Variability Reduction Factor (VRF). The effect of the reduction in variability is included in the overall calculation of the system as a dimensionless factor, because its affect on the return of investment in the BESS is not calculated in a rigid way in the energy market. A reduction in variability will almost certainly guarantee a higher financial return for the project and an increase in variability will almost certainly guarantee the opposite. However, these are determined by the contracts that utilities pay to one another and to plant owners for their energy over a certain period of time, and while they are largely predictable, they do not generally follow a standardized formula.

For this reason, a good generalization is that a decrease in variability will result in an increase in price return on the electricity up to a maximum of the average retail cost of electricity per $\mathrm{kWh}$ in the United States. Then, a reduction in variability from the amount seen without the BESS to zero would yield the maximum variability reduction factor of 1 . Then, the more power the system generated, the greater the return — as calculated simply by the price per kWh. For a clarification on the standardized price per $\mathrm{kWh}$ included in the model of $\$ 0.1031 / \mathrm{kWh}$, please see Appendix A.1 [71]. 
The Variability Reduction Factor (VRF) is given as

$$
V R F=\frac{\left(T_{o t} t_{-} V_{-} V a r\right)-\left(T_{o t} t_{-} S s_{-} V a r\right)}{\left(T_{0} t_{-} P V_{-} V a r\right)}
$$

where Tot_PV_Var is the total second-to-second variability of the photovoltaic output over the sampling period, in kWh, and Tot_Sys_Var is the total second-tosecond variability of the system output with the BESS installed over the sampling period, in $\mathrm{kWh}$. Then the formula to calculate the total system revenue over the lifetime of the BESS is found by first calculating the additional energy price $\left(P_{A d}\right)$ that a BESS could potentially net the PV plant, given as

$$
P_{A d}=P_{R e}-P_{E x}
$$

where $P_{R e}$ is the current average retail price of $\$ 0.1031 / \mathrm{kWh}$, and $P_{E x}$ is the existing price that the PV plant is being paid for the power it produces. Then additional revenue $\left(R_{A d}\right)$ is given as

$$
R_{A d}=V R F \cdot P_{A d} \cdot T o t \_S y s \_O u t-C_{B E S S} \cdot B E S S \_C a p a c i t y
$$

where Tot_Sys_Out is the total energy produced by the system over the operational life of the BESS, and $C_{B E S S}$ is the combined construction, operation, and maintenance costs of the BESS spread evenly over its operational life per kWh of energy storage capacity. BESS_Capacity is the energy storage capacity of the BESS in kWh. The existing revenue $\left(R_{E x}\right)$ is estimated similarly:

$$
R_{E x}=P_{E x} \cdot T o t_{-} P V_{-} O u t-C_{P V}
$$

where $C_{P V}$ is the combined construction, operation, and maintenance costs of the BESS spread evenly over its operational life. 
It is important to realize that, while the calculations of the change in variability and costs incurred by a BESS on a project are reasonably accurate, they are not intended to represent an accurate picture of the entire exact expected financial prospects of a potential project. This is because of the existence of several values that are either unknown or highly variable from one project to the next, including the construction and operational costs of the PV array, and the existing prices paid to PV operators for the energy they produce. In the absence of these values, one can make certain approximations to understand the dynamics of the improvements brought by a potential BESS, but not the exact final value. For example, one could evaluate the prospects of a potential BESS system if one were to say that the operations without the BESS dictated that the price paid to the plant operators per $\mathrm{kWh}$ was half of the average retail price per $\mathrm{kWh}$ (because of the variability of the output); and that in the absence of further government subsidies, a combination of the plant's construction costs spread equally over its operational lifetime and the operational and maintenance costs meant that the plant operated at a $25 \%$ loss.

Therefore the financial considerations included in the model should be seen to serve as an indication of whether a potential configuration could meet or exceed cost-neutrality. To a lesser extent it is also intended as an objective gauge to measure the feasibility of the system configurations relative to one another. The results that include the comparative financial feasibility metrics are included beginning with Experiment 9 in Section 3.1.7.

There are a few other aspects of the model to take note of at this point. The current formulation could conceivably favor a type of battery that is extremely cheap to install and maintain, but that is also highly inefficient. This could happen because the total cost could still work out, even though considerable energy 
would be lost. While it is unfortunate to not be able to fully utilize solar power as an energy resource, in the end, it would be little different than the choice between PV panels of varying quality. With a very expensive, high-efficiency panel, one pays more upfront, but also gets a higher output of energy over time and thus a higher return on their investment. Cheaper, low-efficiency modules could work out just as well in some cases. The energy output might not be as high, however, since their level of investment did not necessitate a higher output the system could be judged a success.

The main caveats to such a scenario are the waste involved with using more materials and the fossil fuel required at present to transport the materials throughout the various stages of the lifecycle. Most of the sources referenced for the costs of the battery types include in their calculation the costs not only of the battery module itself, but also the delivery, installation, maintenance, and end-of-life recycling or disposal. The costs in man-hours and fossil fuel would also be included in these figures, and would likely remain static or increase with a battery type for which the hardware cost was smaller The fact that the battery system as a whole would yield a lower net price indicates that the risk of increased secondary costs are limited. At a certain point, designing a less efficient battery system would require so many batteries that the increased installation and maintenance costs would cause the net price of the system to rise. In that case, the costs calculated by the NetLogo model would be higher than for the slightly more efficient systems. Consequently, the more efficient configurations would be seen as preferred, and the correct balance would be found.

For Experiment 3, the model was set up in BehaviorSpace with the following settings:

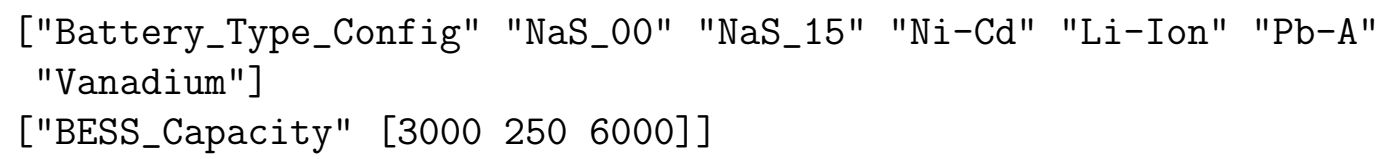


["BESS_SS_Charge" $\left[\begin{array}{lll}0.2 & 0.1 & 0.8\end{array}\right]$

The results of this experiment are tabulated according to the most optimal configurations in Table 3.3. The output of the model when using the optimal configuration is then shown in Figure 3.3.

The results in Table 3.3 show that, according to this model, there are some battery chemistries, such as the NaS_15 type, that less well-suited to grid-level energy storage in terms of their ability to mitigate the variability of solar PV output. This was likely because the additional lifetime charge cycles of the NaS_15 configuration when compared with the NaS_00 configuration only offer comparative advantage when considering their long-term financial feasibility, which was not included in these initial experiments.

\begin{tabular}{lrrrrr}
\hline Rank & VRF & Run \# & Batt_Type & BESS_Capacity & SS_Charge \\
1st & 0.896 & 800 & "Ni-Cd" & 6000 & 0.2 \\
2nd & 0.882 & 233 & "NaS_00" & 5750 & 0.2 \\
3rd & 0.870 & 260 & "NaS_00" & 6000 & 0.3 \\
4th & 0.870 & 694 & "Ni-Cd" & 4750 & 0.2 \\
\hline
\end{tabular}

Table 3.3: Lowest variability configurations from BehaviorSpace Experiment 3

The second thing that became evident with this experiment was that none of the highest VRF values eliminated more than $90 \%$ of the variability. This indicated that the "absolute leveling" application was not behaving properly with these attributes, and that at certain points the output was much more variable than it should have been while using the absolute leveling application. When plugging in the configuration from run number 800 , for example, into the NetLogo model, I found the output in Figure 3.3.

Here, the battery type is set to Ni-Cd, the BESS_Capacity is set to 6000, and the BESS_SS_Charge is set to 20\%. The piece of the puzzle that was missing here was 


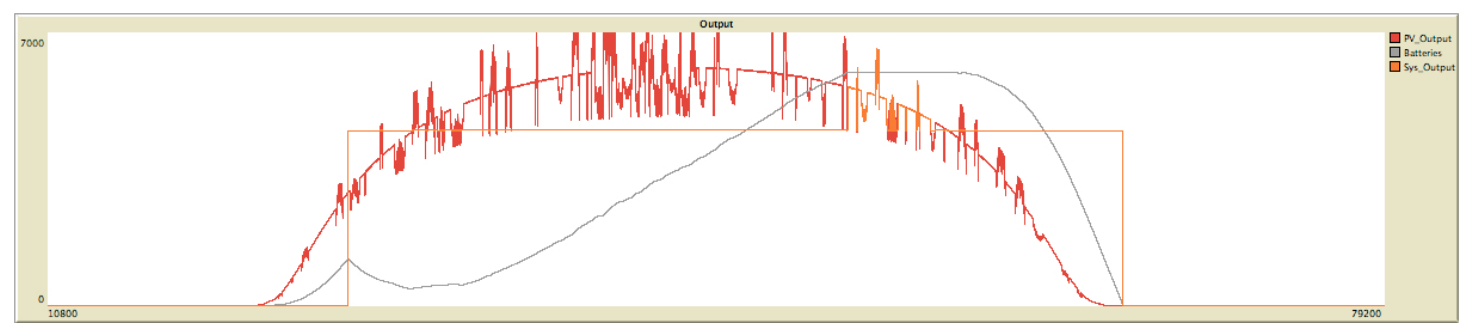

Figure 3.3: Output of an optimal configuration from BehaviorSpace Experiment 3 , showing undesirable spikes in system output during the early afternoon. The values for PV Output and System Output are in units of $\mathrm{kW}$, while the plot for Batteries is in units of $\mathrm{kWh}$, and represents the charge state of the BESS.

the leveling target. Experiment 3 showed that with a certain PV output came the necessity to be able to output a certain amount to the grid. Without the greater energy storage capacities required of an application such as time shifting, the leveling target would need to be set adequately high so that the batteries would not become fully charged during times of peak PV output, when the output of the PV array would be higher than the level of output the system is configured to supply to the grid.

I determined that it was helpful to take the properties that had been optimized thus far and examine them with greater granularity. One of the most important things found in Experiment 3 was the fact that a key relationship exists between the BESS capacity and the leveling target. This was tested further in Experiment 4 .

\subsubsection{Experiment 4}

In Experiment 4, the goal was to include as much of the entirety of the parameter space as possible. I also simplified comparisons by altering the model so that the BESS_Capacity was represented as a percentage of the peak PV output. The SS_Charge continued to be a percentage of the BESS_Capacity. The Leveling-Target was also changed to a percentage of the PV capacity. These were each allowed to vary from .1 to 1 , in increments of .1 . 


\begin{tabular}{lrrrrrr}
\hline Rank & VRF & Run \# & Batt_Type & Capacity & SS_Charge & Lvl_Tar \\
1st & 0.998 & 7969 & "Ni-Cd" & 0.7 & 0.6 & 0.7 \\
1st & 0.998 & 6439 & "Ni-Cd" & 0.2 & 0.5 & 0.7 \\
1st & 0.998 & 8269 & "Ni-Cd" & 0.8 & 0.6 & 0.7 \\
1st & 0.998 & 1339 & "NaS_00" & 0.5 & 0.5 & 0.7 \\
\hline
\end{tabular}

Table 3.4: Lowest variability configurations from BehaviorSpace Experiment 4, showing a high degree of parity in their comparative metrics

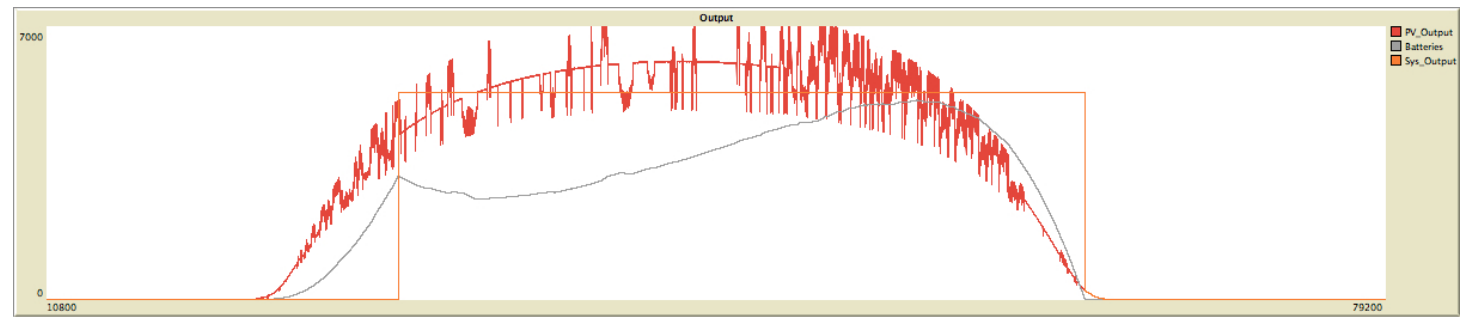

Figure 3.4: Output of an optimal configuration from BehaviorSpace Experiment 4. The values for $P V$ Output and System Output are in units of $\mathrm{kW}$, while the plot for Batteries is in units of $\mathrm{kWh}$, and represents the charge state of the BESS.

Table 3.4 shows the optimal configurations from the model in Experiment 4. Figure 3.4 shows the output of the model with one of the best configurations for the VRF metric, run number 7969. This included a Ni-Cd battery type, with BESS_Capacity set to 0.7, BESS_SS_Charge set to 0.6 and Leveling_Target set to 0.7 .

\subsubsection{Experiment 5}

Several key features of the model were highlighted in Experiment 4. For one, the Leveling_Target was revealed to be squarely optimized at $70 \%$ of the peak PV output. Secondly, the SS_Charge was indicated to be optimized between .5 and .7. The optimal battery type was shown to most likely be Ni-Cd when only considering variability reduction capabilities.

In Experiment 5, the range of parameters used in the search for reasonable configurations was narrowed substantially. I set it to run in two separate instances: one 
with the Ni-Cd battery type, varying the SS_Charge between .5 and .7 in increments of .025, varying the BESS_Capacity between .2 and .8 in increments of .025, and having the Leveling-Target vary between .6 and .8 in increments of .25, to be sure that further resolution was not possible with this parameter. The second part of the experiment would consist of the Li-Ion battery type, and otherwise the same parameter space. I would then iterate this experiment for 7 runs each configuration, in an effort to better account for any unlikely abnormalities that might arise. This amounted to 14,175 runs for each battery type, for a total of 28,350 runs altogether.

It should be noted that the NetLogo version used during this research (version 5.0.4) uses the Mersenne Twister pseudo-random number generator. This number generator works by beginning with a random seed that by default is set at the start of each model run based on the computer's date and time. That is the method by which this and all previous experiments were simulated, yet after Experiment 6 I began to set the random numbers manually to allow better reproducibility. The numbers used for the random seeds are outlined in the sections for the respective experiments.

Table 3.5 shows the optimal configurations of the combined parts of Experiment 5, including both Ni-Cd and Li-Ion battery chemistries. A plot of the model with one of the optimal configurations from Experiment 5 is shown in Figure 3.5. The individual results of the respective parts are included in the Supplemental Data Section A.4.

\subsubsection{Experiments 6, 7, and 8}

Experiments 6,7 , and 8 were focused on refining the way that the model represented certain cloud cover phenomena. First, adjustments were made to account for the fact that the PV array is made up of strings of modules, and that these 


\begin{tabular}{lrrrrrr}
\hline Rank & VRF & Run \# & Batt_Type & Capacity & SS_Charge & Lvl_Tar \\
\hline 1st & 0.997 & 2486 & "Ni-Cd" & 0.3 & 0.575 & 0.7 \\
1st & 0.997 & 9290 & "Ni-Cd" & 0.6 & 0.575 & 0.7 \\
1st & 0.997 & 9857 & "Ni-Cd" & 0.625 & 0.575 & 0.7 \\
1st & 0.997 & 3620 & "Ni-Cd" & 0.35 & 0.575 & 0.7 \\
\hline
\end{tabular}

Table 3.5: Lowest variability configurations from BehaviorSpace Experiment 5

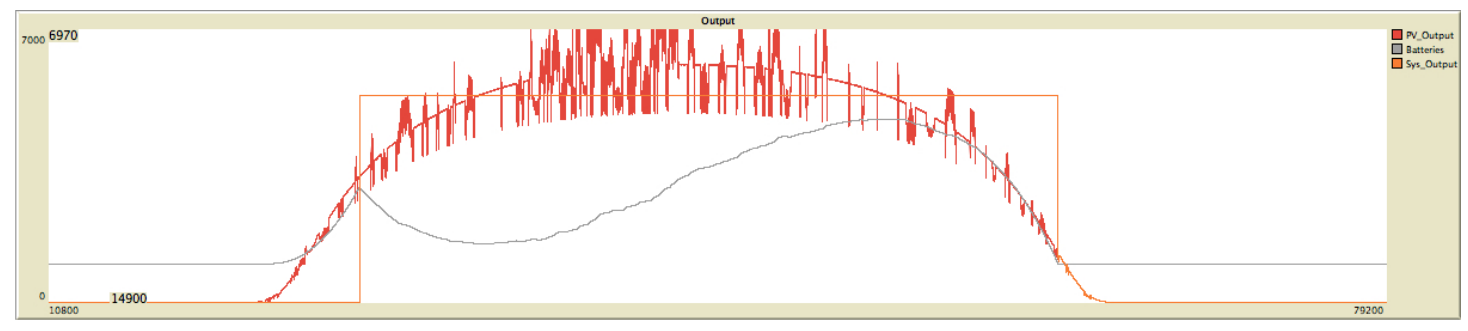

Figure 3.5: Output of an optimal configuration from BehaviorSpace Experiment 5 ; this configuration was the most optimal of the Li-ion configurations, though not the most optimal overall, and is included for comparative purposes (this output represents a $V R F$ value of approximately 0.994$)$; notice the system output defaults back to that of the PV array when the battery is discharged to its minimum level, causing somewhat increased variability; the values for PV Output and System Output are in units of $\mathrm{kW}$, while the plot for Batteries is in units of $\mathrm{kWh}$, and represents the charge state of the BESS.

strings tend to behave as units because they are wired in a series. A string will often actually drop to zero output (or at least a voltage level below the minimum requirements of the inverter) when the string is partially covered by a cloud, because of the enormous internal voltage difference within the modules. The model was updated to reflect this, and some additional controls were added for the nominal voltage of the inverter - which determines the number of modules in a string. This change did not substantially affect the comparative metrics for variability reduction, which were taken over entire days and thus largely averaged out this effect.

These experiments were also used to refine the calculations for the comparative financial feasibility metrics for each BESS configuration, and those metrics are included in the results of the following experiments. 


\subsubsection{Experiment 9}

Experiment 9 was set up to simulate the model over a wide parameter space, and to increase the number of runs to 25 to reduce the uncertainty related to random deviations of the system. The setup for this experiment is given as:

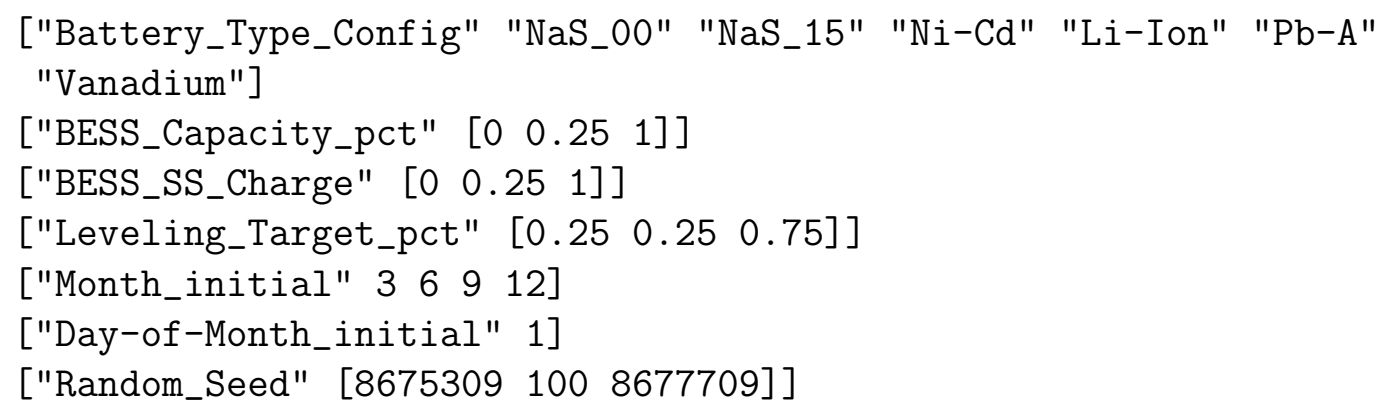

The total number of runs was 45,000, as this took into account 25 runs for four days over the course of a year in which each possible configuration was simulated. The results of this experiment are given in Figure 3.6. An expanded look at these results for the NaS_15 battery type, which was one of the most feasible types found in this experiment, is given in Figure 3.7. An analysis of these results is presented in Section 4.1.

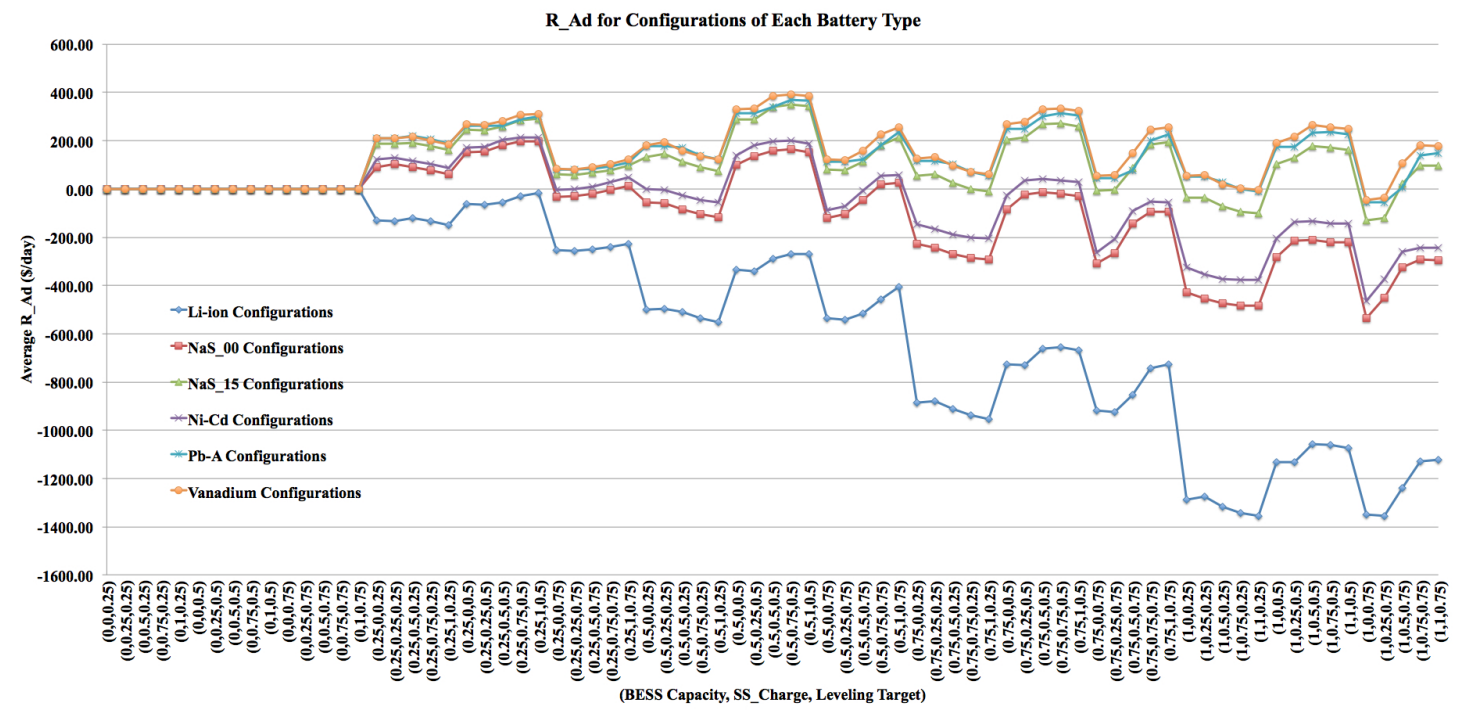

Figure 3.6: Experiment 9 results showing $R_{A d}$ values for different configurations with each battery type 


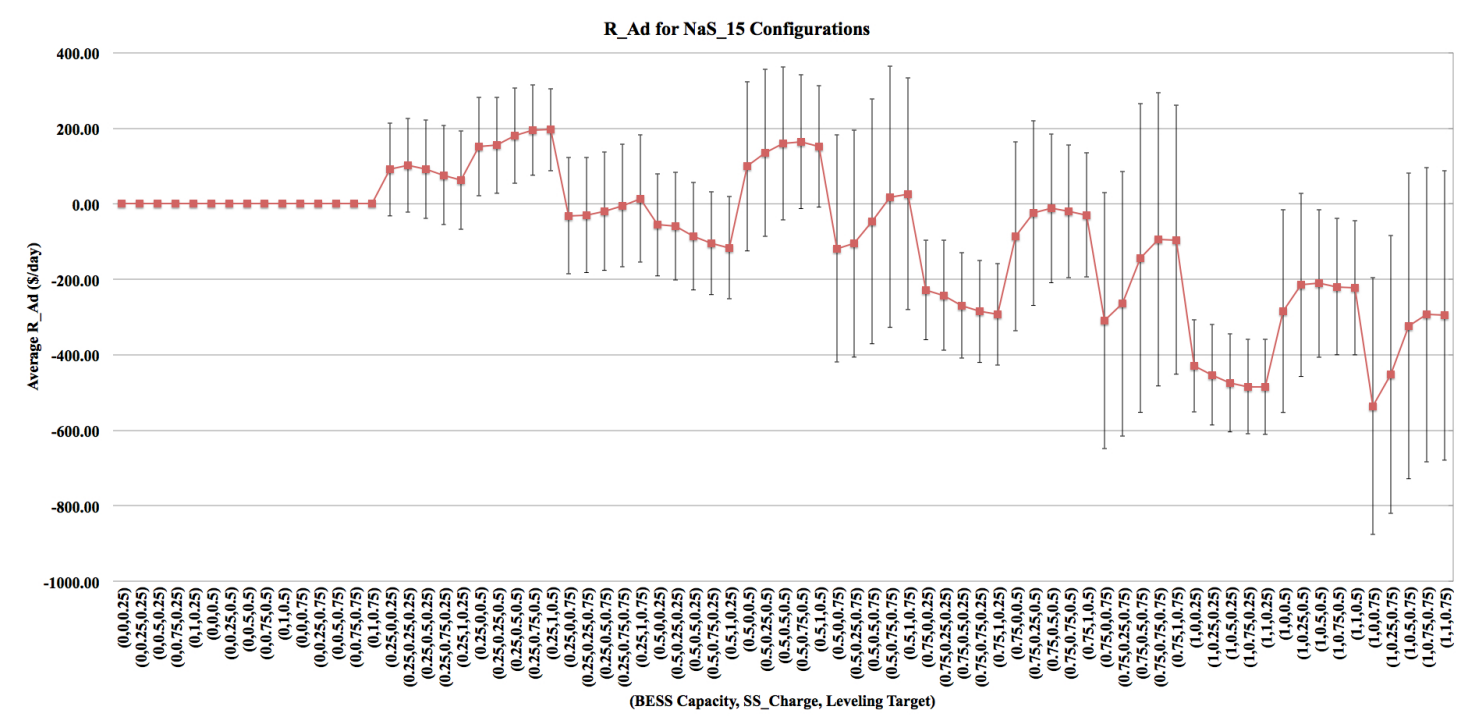

Figure 3.7: Experiment 9 results showing $R_{A d}$ values for different configurations of the NaS_15 battery type, including the standard deviation from the average across 25 runs with each configuration

\subsubsection{Experiment 10}

I set up Experiment 10 to illustrate how a completely different set of random seeds would affect the outcome of the system. In order to see the effects at a level of comparable scope to the results included in the thesis, I used the same setup as Experiment 9, except for the random seeds - which I varied between 5000 and 7400. The Random_Seed variable was therefore set by:

["Random_Seed" [ [5000 100 7400] $]$

This experiment totaled the same number of runs - 45,000 — as Experiment 9. The results of this were nearly identical to the results of Experiment 9, and thus are not shown here. This indicated that the different feasibility metrics resulting from different configurations are largely independent of which set of random seed values are chosen, when averaged over at least 25 runs.

\subsubsection{Experiment 11}

In Experiment 11, I narrowed the parameter space of interest to the regions that seemed to consistently contain the most feasible values for the parameters. The 
SS_Charge parameter seemed to have much less of an effect overall on the $R_{A d}$ value than the other parameters, and so in this experiment I set it at a constant 0.5. I then limited the battery types to the three most feasible from above, and limited the parameter space for the BESS_Capacity_pct and Leveling_Target_pct to the region from 0.3 to 0.7, (in increments of 0.1). The setup for Experiment 11 consisted of the following:

[Battery_Type_Config" NaS_15" "Pb-A" "Vanadium"]

["BESS_Capacity_pct [ $\left[\begin{array}{lll}0.3 & 0.1 & 0.7\end{array}\right]$

["Leveling_Target_pct" [ $\left[\begin{array}{llll}0.3 & 0.1 & 0.7\end{array}\right]$

["BESS_SS_Charge 0.5]

["Month_initial" 369612 ]

["Day-of-Month_initial" 1]

["Random_Seed" [8675309 100 8677709] ]

This experiment totaled 24,300 runs, with the same arrangement of days of the year, and the same random seeds. The results of this are shown in Figure 3.8.

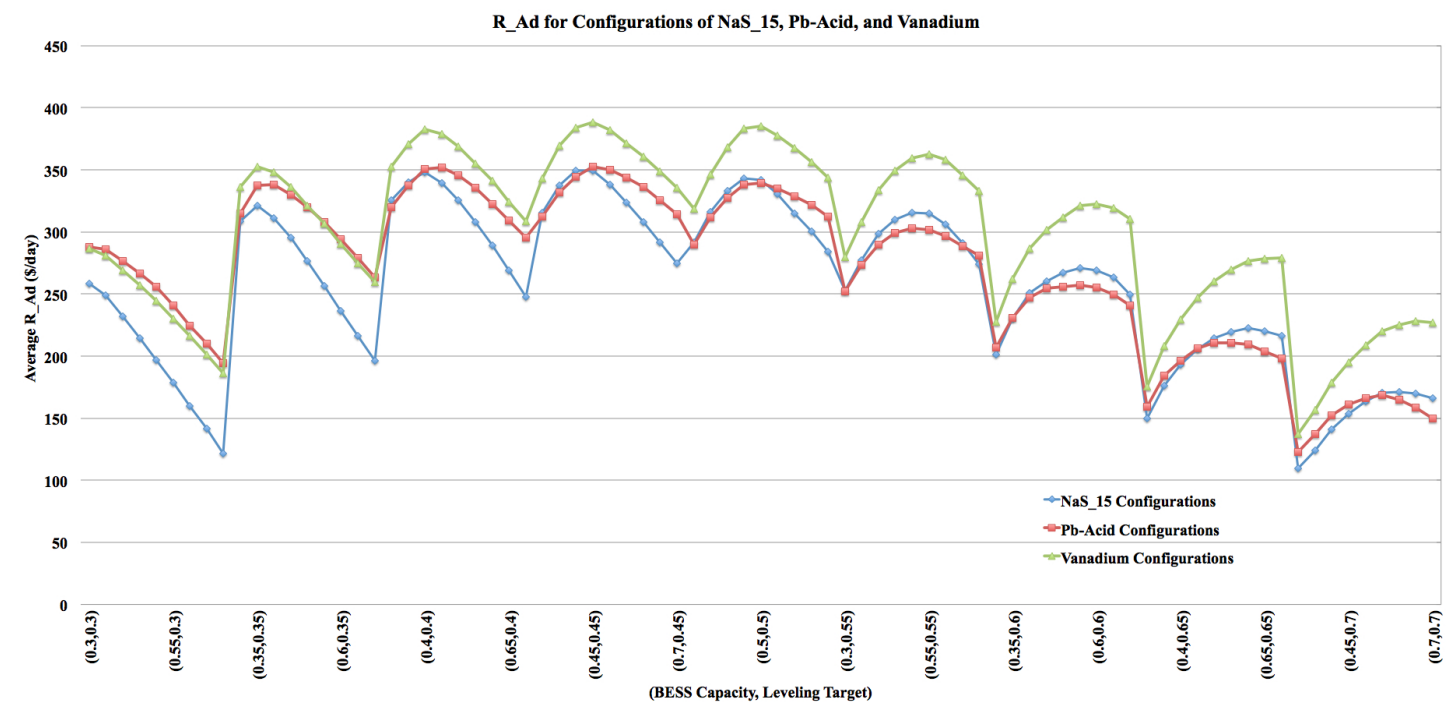

Figure 3.8: Experiment 11 results showing $R_{A d}$ values for each configuration in the narrowed parameter space of the battery types NaS_15, Pb-A, and Vanadium; the regular intervals represent the relationship between the BESS capacity and the leveling target, with multiple configurations yielding approximately the same average additional revenue 


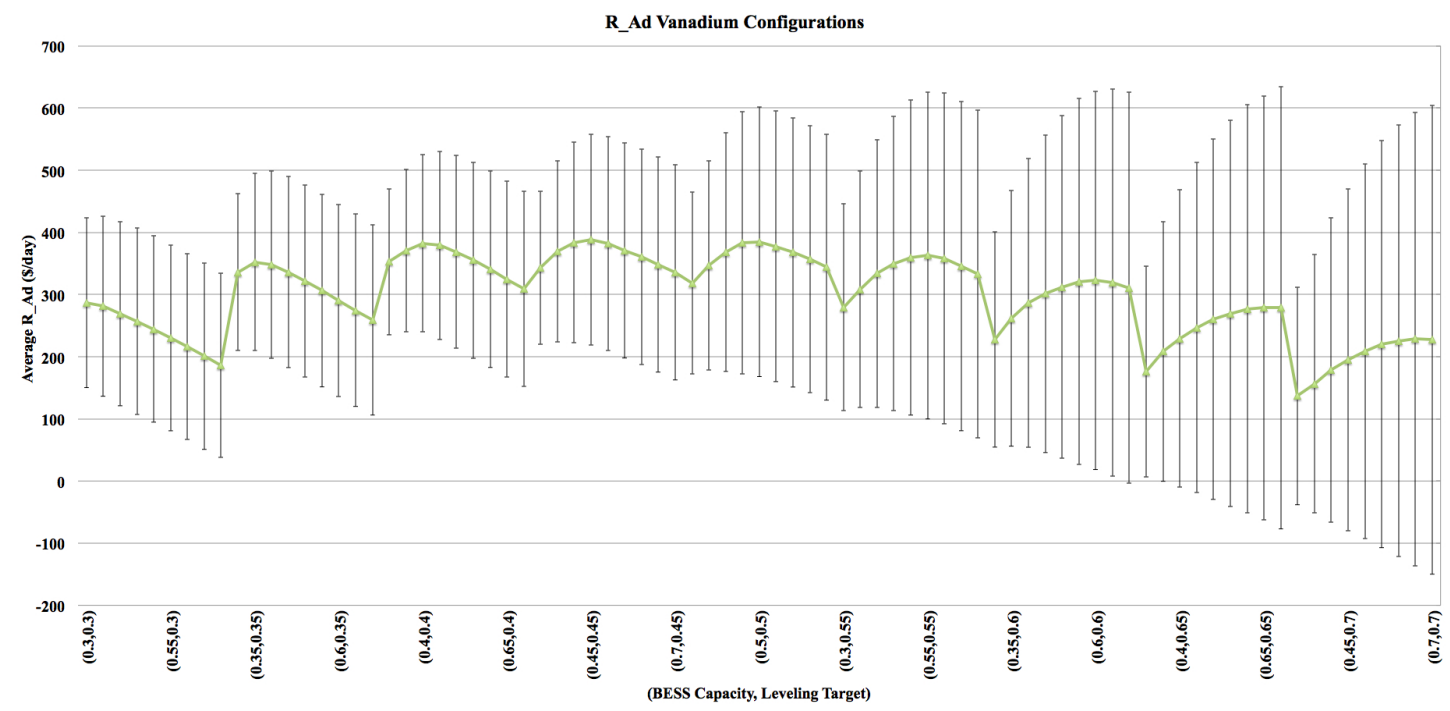

Figure 3.9: Experiment 11 results showing $R_{A d}$ values for each configuration in the narrowed parameter space of the Vanadium battery type, including the standard deviation from the average across 25 runs with each configuration; the increasing standard deviation as the leveling target increases past $50 \%$ of the daily peak PV output corresponds to the potential for both greater financial gains and losses on a daily timescale when increasing the leveling target of a BESS with a higher energy storage capacity 


\section{Discussion and Conclusion}

\subsection{Analysis and Interpretation of Primary Results}

In general, the results of the BehaviorSpace experiments reveal some important relationships between the parameters of the system, as well as several tendencies of the system as a whole. They point to a general range of parameter settings as the most likely to be feasible, and these are outlined in the following sections.

\subsubsection{Optimal Battery Chemistry}

The most relevant metric was $R_{A d}$ because it took into consideration the costs of the battery, which were well-defined, and not the costs of the PV array, which were less well known. It therefore provides the most information while relying on the least assumptions. The VRF metric, while informative, only looks at the capabilities of a battery chemistry on a day-to-day basis and not over its operational life. This is why Ni-Cd was chosen as the optimal choice by VRF, but not by either of the other metrics - because it is arguably better than NaS in the short-term. The total improved revenue metric $\left(R_{\text {Tot }}\right)$ was useful in looking at whether or not a BESS could make a project revenue neutral overall. However, because that is usually not the primary criteria for the inclusion of a BESS in a system, and because of the additional assumptions and approximations made with regard to the PV array costs and revenue in the calculation of this metric, it is also seen as less relevant overall than the $R_{A d}$ metric.

The NaS, Pb-Acid, and Vanadium battery chemistries were seen to be the optimal types when looking at the average $R_{A d}$ metric that resulted from their most 
feasible configurations. This was observed to be related to the fact that NaS batteries, as included in the model, are second only to lead-acid batteries in low cost, while being second only to Li-ion batteries in efficiency. They are also capable of more operational cycles than most battery types when discharging to no less than $15 \%$ each cycle. Overall, based on the experimental statistics for NaS batteries included in the model, it was the battery type that seemed most likely to be chosen by the model as the most feasible, and this was indeed the outcome.

\subsubsection{Feasible Steady State Charge Based on Predicted Variability}

In general, changing the SS_Charge metric seemed to cause less change in the $R_{A d}$ value relative to changing the BESS_Capacity_pct and Leveling_Target parameters. This is evidenced by the fact that the $R_{A d}$ values through each cycle of the five SS_Charge values in Experiments 9 and 10 are relatively steady, but there is a noticeable shift in not only magnitude but overall behavior of the $R_{A d}$ value when the leveling target is changed. A shift of similar magnitude seems to be observed when the battery capacity is changed.

While the changes in $R_{A d}$ due to changing SS_Charge were not substantial, they did form small patterns in some cases. These were observed to be related to several types of nuanced phenomena. In the preliminary experiments that were run when the model was being designed and tested, it seemed that one could make a case for setting the steady state charge reasonably high so that the batteries would have time to be charged adequately before the PV output was high enough in the late morning to make up the majority of the overall output. This notion was revealed to underestimate the effect of the late afternoon peak demand. Because demand is higher in the afternoon, the leveled output would be shifted that direction regardless of the steady state setting, because of the way the model was set up. The phenomena that occurred whereby the battery would be completely saturated with charge before the PV output dipped back below the leveling target 
occurred far more often than the batteries not having enough charge.

In general, the optimum setting for this parameter was observed to depend the most on the weather patterns at the site of any of the parameters. The results of the later experiments, which were run over 25 random amounts of cloud cover for different days of the year, show that while the average $R_{A d}$ values for the different steady state values were relatively close together, the standard deviation of the values used to find this metric for each configuration increased with the increasing steady state charge setting. One could then infer that when the system operators increased the level of charge the BESS would need to reach each day before it could begin to output to the grid (i.e. the steady state charge), they would potentially increase the return on their investment, but also the risk that the irradiance would not be as intermittent that day as they had predicted, and thus that the batteries would become fully charged at times that they would've otherwise been able to accept excess energy from the array. This would then cause the output to spike, thereby increasing the variability of the output significantly. The best value for this parameter was, therefore, seen to be largely dependent on the characteristics of the cloud cover for that day, and so an ideal setup would perhaps automatically adjust this value for the BESS each day, depending on the weather patterns in the area.

\subsubsection{BESS Energy Capacity and Leveling Target}

Beginning with Experiment 4, the experiments showed that increasing the BESS energy capacity of the system did not necessarily increase its feasibility. The assertion here is that when the leveling target is set too low, the model prefers a larger BESS capacity to compensate, and yet this still does not provide adequate coverage and the variability mitigation if the system falters. However, when the leveling target is set a bit higher, the target is easier to maintain because the correct balance is found between leveling and what would more properly be referred 
to as peak shifting. This is certainly a more efficient means of maintaining a level output, and lends further credence to the fact that a time shifting application should not be considered the primary directive of electrochemical energy storage, because of the significant capacities required - though it can be useful as an auxiliary service during overcast days.

Experiment 5 furthered the notion that an optimized system is one where the design choices of the system (i.e. PV and BESS capacity) are such that they provide ample parameter space for daily optimization and increased performance, without creating wasteful spending in construction and maintenance costs by "over-specing" the project. Experiment 5 found the ideal BESS energy storage capacity to be between $50 \%$ and $75 \%$ of an hour of peak PV power output - meaning that an optimally sized system could store enough energy to compensate for the entire output of the PV plant at peak output for between 30 and 45 minutes. The later models confirmed this, and found that the BESS capacity that is most feasible is most often in the range of 50-60\% of the hourly peak power output of the array. This value was not seen to be constant across the different battery types and leveling target values used, however, and It seems that the $R_{A d}$ value attainable by using a certain type of battery depends on a certain ratio of the battery energy capacity to the leveling target. While this tendency is not independent of the steady state charge, that parameter was seen to have relatively less of an effect on the resulting $R_{A d}$ value.

It is furthermore difficult to know exactly how the capacities examined here compare to those found in the industry. Many BESS systems are tied to a particular substation and are operated by a utility for frequency control and other grid support services, and are thus not sized directly proportional to a single plant PV or otherwise. Some other examples of these systems (such as the previously mentioned BESS at the Laurel Mountain project in West Virginia), are integrated 
directly with the plant, and in that example the power capacity of the BESS is approximately $33 \%$ of the power capacity of the wind farm. It is important to note, then, that the potential BESS configurations outlined in this thesis assume a maximum power output of the BESS equal to that of the PV plant to which it is tied. The BESS energy storage capacity is then quoted throughout this thesis essentially in terms of the length of time the system could match the peak PV power output.

While the later experiments in this thesis were performed using random seeds for reproducibility, it would be interesting to see the outcomes of the searches when completely random values were used for each run. The highly deterministic results of the experiments do not do justice to the extremely stochastic nature of the system, and instead are indicative of the fact that for each run of a configuration of the model that was performed, the effects of cloud cover were represented as random over the course of the day - yet for the sake of reproducibility the different configurations were run over the exact same type of "random" cloud cover.

A perfectly equivalent $R_{A d}$ value is not necessarily possible for all BESS capacities in this range by simply varying the leveling target, but very nearly so. This is to be expected, however, because one can get the same leveling benefits from a smaller BESS if the power output to the grid is sufficiently low. The biggest change shown in Experiment 11 among the different capacities is not necessarily in the average $R_{A d}$ over the days measured, but in the standard deviation of the average $R_{A d}$ over those configurations. This stands to reason as well — increasing the size of the BESS for a given PV array increases the potential for both financial gains and losses, depending on the weather patterns for each particular day.

Within the scope of the results of the narrowed parameter space of Experiment 11, the model seems to predict reliably what the general regions of feasibility are, 
according to the technological and financial specifications used in the model, but when narrowing in on those regions, the resulting $R_{A d}$ values show a great deal of parity, indicating that within the scope of those regions, the different configurations are seen to not provide a change in $R_{A d}$ values consistently beyond the level of statistical uncertainty inherent in the system.

In each of the experiments that were run within the BehaviorSpace environment, results indicated certain configurations that would give the least variability in the output of the system, and would create the most additional revenue based on the estimated costs of the systems and the estimated improved prices for the energy produced. Because the experiments were run using the Absolute Leveling application, what this meant in a general sense is that the model would vary the charge and discharge characteristics until the output from the battery covered the daily output from the PV array itself. In the plot of the output, particularly in Figure 3.2 , one can see that the value of the steady state charge that is chosen determines when the battery will begin to output to the grid. Too low a value, and the batteries will deplete before the PV output is greater than the leveling target. Too high a value, and the batteries will be completely charged before the average PV output gets below that of the leveling target, and the output of the system will spike as a result.

On the other side of this balancing equation is the leveling target. A higher leveling target means that the steady state capacity can be higher without risking the batteries becoming fully charged during peak hours. However, this can mean that the batteries are depleted too early in the evening. Since the design of the Absolute Leveling application is such that there is near-zero variability across the output period, the output at the very end of the day when PV output is minimal is expected to be exactly the same as during the midday sun, when the PV output is at at its peak. Thus, with no way to gradually decrease the system output at 
the end of the day, if the batteries do not have sufficient reserve capacity from earlier in the afternoon they will discharge to their minimum level too early, and the system output will drop from the leveling target to the level of the last vestiges of the PV output — reintroducing its unmitigated variability to the system output - before going to zero. In practice this would not increase the average variability of the system substantially more than that introduced to the grid from a particularly large distributed solar array in a residential or commercial setting with no battery storage. However, these occurrences would add considerably to the variability of the output compared to its output outside of these situations, which would otherwise be very close to zero. For this reason configurations that would create such a scenario were excluded from the BehaviorSpace results.

\subsubsection{Optimality and Robustness}

The goal of running the BehaviorSpace tool was to find the parameter configuration that yielded the lowest possible average variability for the system's output, and later to find the highest measure of increased revenue from a BESS. This was found easily, yet when these configurations were run in the model, they did not consistently give the same results. In fact, when attempting to replicate the output from earlier results, the model gave an output with average variability that was several orders of magnitude higher than that from the BehaviorSpace results. Typically this increased variability was because of the nature of the model discussed previously — which is that when the steady state charge level is set too high and the leveling target is too low, the batteries become completely charged when the PV output is still above the leveling target. This effect is shown in the output in Figure 4.1.

Here, the same configuration is shown creating a different output because of the inherent randomness of the system caused by weather effects. The reason that this occurred some times and not others was because of the randomness built into the 


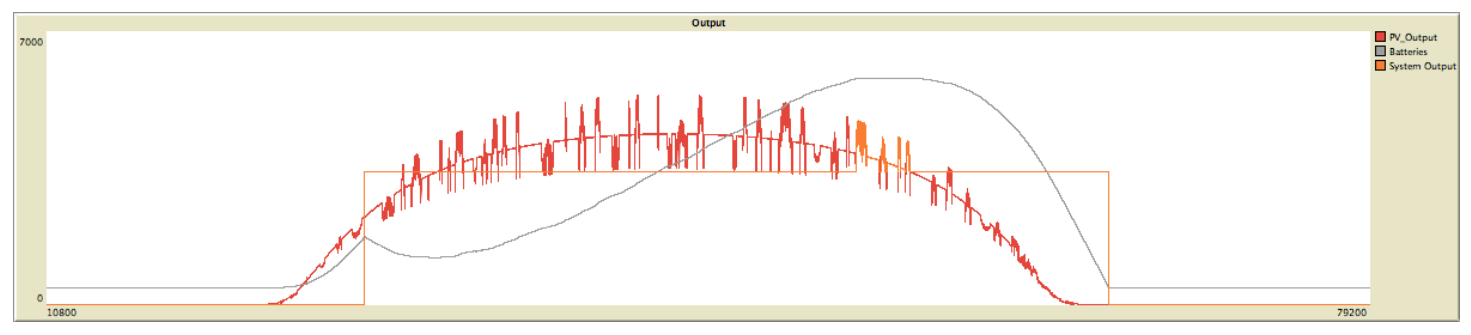

Figure 4.1: Suboptimal output of optimal configuration from Experiment 2

model to account for the inherent unpredictability of the effects of weather on the output of the system. When one approaches the extremes of the system, one is more likely to encounter drastically different outcomes from one run to the next. Because both local and global optima are, by definition, extremes of the system, this is to be expected. The randomness in the model was built on a random seed generator that in the earlier experiments was determined by the value of the system clock at the time of the model run to determine the value of the various random numbers in the system. In the real world, these random factors would amount to changing weather patterns on a day to day basis, and the random formation and movement of clouds and atmospheric particulate matter on a minute to minute basis.

Thus, a system configured with the parameters used to find the initial results would be considered a highly optimal system, yet not a robust system. The optimality of a system depends on how well suited it is to perform under a certain set of conditions. The robustness of a system depends on how well suited it is to the effect of changing conditions. Unfortunately, these two qualities are often mutually exclusive in man-made systems.

Therefore, a search for reasonable configurations for this system will be somewhat more complex than that for other non-linear systems. The main question is how much should the configuration of the parameters compensate for the uncertainty presented by changing weather systems. In a practical sense, this is often 
accounted for by a certain level of excess capabilities built into the system. This reserve is determined by the theoretical limits the system will see under normal operating conditions, and those theoretical limits can be derived mathematically from the algorithms governing the model components. These limits can then be tested in a software environment such as NetLogo. In fact, one might say that this is the area in which a "brute-force" method of testing a set of parameters repeatedly can instill confidence in a chosen reserve capacity. The risks and limitations of this approach are evaluated further in the following section.

\subsection{Limitations and Opportunities for Future Improvement}

There are several limitations to the results found throughout this research that have been mentioned separately, but should be thought of collectively. The first and most general of these is the fact that a potential BESS modeled using the method outlined here exists as an isolated system for the sake of simplicity and clarity of the calculations, yet that is not the reality of the situation in an actual implementation of this system in the real world. The important effects of the external systems a BESS would typically interact with have been included as approximated variables in this model — such as the market price for energy or the structure of the grid in the region the BESS is located. While this is generally a reasonable approximation, these values are arrived at as placeholders for important phenomena rather than substitutions for the actual value. By this, I mean that the accuracy of the financial feasibility calculations are not sufficiently accurate in their current form to exist as the sole measure of workability of a potential system - yet they create a very clear picture of the potential capabilities of such a system with regard to its primary function ${ }^{1}$, which is to mitigate the variability of the attached PV array.

\footnotetext{
${ }^{1}$ This is the primary function of most systems of this kind that are attached to the grid at the same substation as a PV array.
} 
As mentioned previously, many of the approximations of the model lie in the financial calculations. While the cost of a BESS is fairly well-documented in academic and industry literature, the return price on energy is much less deterministic. It is due to a number of factors ranging from the daily market price at the regional grid interconnect to the national, state, and municipal subsidies paid to renewable energy developers. The approximation of this used in the model is simply related to the national average retail cost of electricity for 2010. Various levels of improvement to this value could be implemented. One approach could be to perform a rudimentary predictive algorithm for the real-time energy cost at the grid interconnect based on archival data for that point in time during past years. Depending on the timescale of data used, one could then have the model take into account seasonal differences in desirable system configurations, as well as daily or hourly differences.

The manner in which the system output is "leveled" is rather simplistic. It is meant to show the absolute capabilities of a potential BESS to compensate for certain levels of fluctuation in PV output, though it lacks nuance in a few ways, and the implementation of such an application in the real world will usually reflect the price of energy at each time of day. For example, a utility will likely desire to output all available energy to the grid in the morning when demand is typically higher - even if the output is more variable this way - and then wait to charge the BESS until closer to noon. This is reflective of the myriad ways that such a system could be configured when considering the dynamics of the modern electric grid, shows the considerable opportunity for improvement of the model in terms of how well it can represent those various scenarios.

Finally, the tool used to simulate the model might be seen by some as a type of limitation because of the time required for large experiments. The reasoning behind using NetLogo to build this model versus a command line implementation 
was related to the fact that the UI is easily customizable, and that it provides a relatively intuitive interface for people that may want to know more about the feasibility of a potential BESS, but that might not have a great deal of experience with electrical engineering or programming. Ultimately, since the original goal of the model was to have a tool that could be quickly and easily configured to show a "what-if" picture of a particular BESS configuration, the runtime of the model was not a primary design concern. After seeing the potential for more accurate optimizations, however, it is clear that a more traditional scientific simulation platform such as MatLab or LabView might offer some of the same benefits of NetLogo while also providing improved runtime. It would be interesting to see if implementations on those platforms would make further research with the model more efficient.

\subsection{Effect of PV Dispersal Area on Average Variability}

One of the ancillary findings of this thesis was that, based on an analysis of the PV data from Oahu, increasing the size of the PV area (by controlling which solar modules were included in the data of interest) did not generate results indicative of a reduction in net variability. ${ }^{2}$ The possible reasons for this are given below. This was in contrast to the findings of several papers on the subject, including $A$ Simple Cloud Simulator for Investigating the Correlation Scaling Coefficient Used in the Wavelet Variability Model, by Matthew Lave and Jan Kleissl, where the authors described one of the primary results of dispersing a PV farm as reducing the average variability of its output [72]. Specifically, they note that

... six times less reserve resources are required to mitigate fluctuations for a distributed plant over $20 \times 20 \mathrm{~km}$ than would be required for a central plant of the same power capacity.

\footnotetext{
${ }^{2}$ It should be noted that this aspect of the data analysis was merely incidental to the main focus of the research, and as such was less thoroughly examined than the main body of the research - though some of the results may provide insight into exceptions to the notion that a larger area equals less variability.
} 


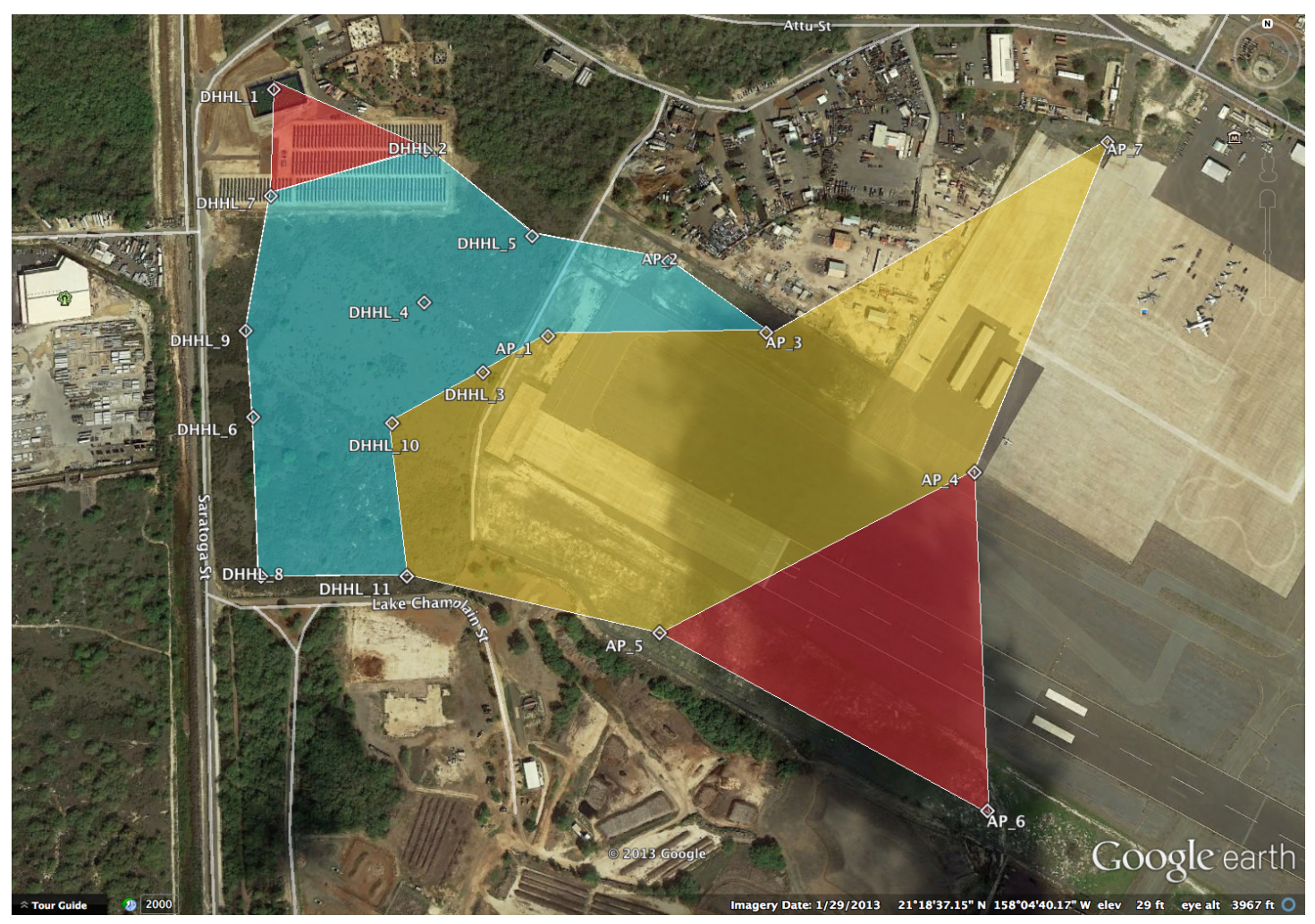

Figure 4.2: Map showing the regions of interest from the NREL Oahu site

In Figure 4.2, the solar modules used to gather the data for the NREL Oahu MIDC datasets are shown, and are connected to form three shaded regions. In this map, the blue area is contained by the yellow area, which is contained by the red area. During my analysis, I first removed the data from modules constituting the extremities of the red region because they represented solar tilt, which was outside the scope of my study. Then, in an effort to better represent the scope of the area covered by the Outback solar project in Oregon, which I was comparing my data to, I removed the data from the modules constituting the yellow region. I was then left with the blue region to conduct the comparative study with, though it was from these preliminary datasets that I concluded that the second-to-second variability over the year did not change significantly from one region to another.

The differences between my results and those found in the aforementioned paper could be due to a number of different factors. Most notably, the irregularity 
of the geometry of the areas that were included in this thesis, especially relative to local weather patterns. One might imagine that if one expanded the area of a site, and the vast majority of that expansion was parallel to the normal direction of cloud cover in the area, then the variability might not be alleviated and indeed could actually be worsened. This would be because of the lack of PV panels outside the path of a cloud continuing to accept solar radiation, while those under the cloud diminished in power output. If there were simply more modules lined up under the average direction of the movement of the clouds, then there would be less of a chance that the cloud cover would miss the panels entirely.

While the macro-level weather patterns in Hawaii generally form a stable Northeastto-Southwest gradient, the Southwest side of the island that this site is on is often affected by the canyons that form the central part of the island. I concluded therefore that a more in-depth analysis would be required to ascertain whether the irregular geometry of the included area was a factor in the absence of a reduction in variability at different scales. It should be noted at this point that the NetLogo model outlined throughout the thesis represents the clouds passing over the PV array in a deterministic way based on the geometry of the array (approximated as a square area) and the geometry of each cloud (approximated as a circle) which are then represented to move in a consistent direction - though this direction is unspecified. An interesting opportunity for future research may be to add more detail to the manner in which the model relates the geometry of the array with the cloud cover passing over it, and to see if those parameters can be adjusted to replicate the lack of change in variability observed over different areas as has been seen in the Oahu data. 


\subsection{Role of Infrastructure Integration}

One of the foremost papers in past few years on the subject of the integration of renewables into the electric grid is the paper prepared for NREL by GE Energy titled Western Wind and Solar Integration Study. It is as rigorous as it is comprehensive, outlining not simply the obstacles to further integration of stochastic energy resources, but also novel ways to solve those problems. One of their major findings echoes that from the paper previously mentioned in that the key to further integration is geographically broad synchronization. Their footprint is, of course, larger than the previously mentioned study — they evaluate the difference in variability from a municipality area all the way up to the Western Interconnect. In doing so, they show that adequate communication systems and short response time devices can have a staggering impact on the variability, often reducing variability tenfold or more over the course of a year [21]. Batteries are not explicitly evaluated in the study, because, as the authors briefly acknowledge, at present the energy losses incurred for a BESS using traditional battery chemistries average about $25 \%$. They point out that many methods of spinning reserve currently used contribute less waste energy than this, and from an economic point of view provide the same service with less R \& D overhead.

This is a valid point, and a perspective that many have adopted. However, wasted energy from current methods of maintaining spinning reserve is often in the form of natural gas or other fossil fuels. While this is discussed in the NREL paper, it is not seen as a primary hindrance by the authors of the paper. Furthermore, the premise of the NREL paper is not to find ways to completely adopt wind and solar as our primary, base load generators, but instead to find ways to allow for integration up to $35 \%$ (including 30\% wind and 5\% solar PV and solar thermal) $[21,73]$. This is tied directly to ongoing initiatives in several states to reach a similar figure of overall generation by a specified date — such as $33 \%$ by 2020 in 
the case of California. The NREL paper outlines a means to achieve this power generation reliably through a variety of common-sense approaches to forecasting, communications, demand response, and infrastructure improvements [21]. These are excellent steps, but the question remains of how the grid will cope with additional renewable integration after we reach that important first milestone of $35 \%$. That is precisely when batteries will be required, if not sooner.

\subsection{Conclusion}

This thesis has shown that though the path forward is not without questions, there is certainly a place in our future for grid-scale battery energy storage systems. The simulation and analysis of a PV array connected to such a system has echoed here what has been shown elsewhere before, which is that the precipitous decline of the price of photovoltaic cells combined with the aggressive progress of new technologies such as NaS and Vanadium redox flow batteries (as well as the evolution of older ones, such as lead-acid cells), has opened up entirely new areas of research and development in the field of renewable energy. The findings of this thesis are that existing grid-connected PV systems are likely to be brought closer to profitability by installing a BESS. It was observed that different costs seem to play different roles at different scales, and that the return on investment will vary widely by region.

Based on the specifications included in the model for the different battery technologies, the sodium-sulfur, lead-acid, and vanadium flow battery types were consistently seen to be the most feasible, when considering the factors that would be the most important in such a design decision in terms of a potential system's variability mitigation capabilities as well as its initial and operating costs. The steady state charge was seen to have relatively little impact on the average longterm feasibility when each simulated value remained unchanged throughout the year. 
Further analysis is required to know if this is because of an overall lack of impact on the daily variability, or if on a yearly timescale, the days that a static steady state charge is optimized and the days that it is less than ideal balance each other out to cancel any but a relatively small effect on the comparative metrics.

It was then observed that the average longterm feasibility of the system was tied to the relationship between the BESS energy storage capacity and the leveling target. When considering the three battery types mentioned above, the configurations that resulted in the highest additional revenue with the least variability were consistently those that used a BESS energy capacity of approximately $50 \%$ of the hourly peak power output of the PV array, and a power output leveling target of approximately the same. This was seen to have a large variation over the samples taken, with a standard deviation much greater than the difference between the average additional revenue of this configuration and other similar ones. However, when the experiments were performed with entirely different random seeds (i.e. entirely different weather patterns) the same configurations for each of battery type were seen to be the most feasible, with small changes in relative magnitude between the configurations. This indicated that when the comparative metrics were averaged over a year or longer, the variation due to the different levels of stochasticity on different days is relatively less than the variation due to seasonal differences in additional revenue per day.

Battery technology is still no doubt in its nascent stage. Though some forms of the battery have been around for a number of years, new chemistries and methods have reopened problems that were once thought unsolvable. The issues facing variability mitigation are, at their heart, the issues facing energy storage. Better forecasting, communication, demand-response, and infrastructure integration will certainly go a long way; as will demand-side best practices that have been enabled by the evolution of smart grid technologies. While important, these all 
simply temporarily alleviate in some way the core issue which is that we must be able to store energy in some form if we are to escape from a reliance on fossil fuel consumption. The best logistics companies in the world need warehouses to store their goods between shipments - regardless of how fast or efficient they are. This is exactly what energy storage means to the grid - to not have to let natural gas plants idle continuously as reserve for when the wind calms or a cloud passes overhead. It also means security and dispatchability. Solar arrays of the future will be built not only in locations with extreme amounts of sunshine; and the savings in costs associated with infrastructure maintenance will begin to grow as we begin to harness energy closer to where we use energy. Energy storage is the vehicle that will allow us to move in this direction, and soon the leap to embrace the capabilities it offers will not be a leap of faith at all — it will be based on proven results. In many ways it already is. 


\section{Bibliography}

[1] J. P. Rodrigue et al. 2013. "Liner Transatlantic Crossing Times, 1833 - 1952 (in days)." The Geography of Transport Systems. Hofstra University, Department of Global Studies \& Geography. http://people.hofstra.edu/geotrans/eng/ch3en/conc3en/linertransatlantic .html, part of http://people.hofstra.edu/geotrans.

[2] Donald J. Wuebbles and A. K. Jain. 2001. "Concerns about climate change and the role of fossil fuel use." Fuel Processing Technology. Volume 71. Issues 13. June. Pages 99-119. ISSN 0378-3820. DOI: 10.1016/S0378-3820(01)001394.

[3] Daniel A. Lashof and Dilip R. Ahuja. 1990. "Relative Contributions of Greenhouse Gas Emissions to Global Warming." Nature. April 25. Volume 344. Issue 6266. Pages 529-531. DOI: 10.1038/344529a0.

[4] EIA. 2012. "Energy Explained: Your Guide To Understanding Energy." U.S. Energy Information Administration. Last modified May 25. http://www.eia.gov/energyexplained/index.cfm?page=renewable_home

[5] Jordi Bruno and Rodney C. Ewing. 2006. "The Nuclear Fuel Cycle Environmental Aspects: Spent Nuclear Fuel." Elements. December. v. 2, p. 6: 343-349. DOI: gselements.2.6.343.

[6] Donald Mitchell. 2008. "A note on rising food prices." World Bank Policy Research Working Paper Series, Vol 4682. July 1. http://ssrn.com.proxy.lib.pdx.edu/abstract $=1233058$

[7] Allan L. Drewitt and Rowena H. W. Langston. 2006. "Assessing the impacts of wind farms on birds." Ibis 148, no. s1: 29-42. DOI: 10.1111/j.1474919X.2006.00516.x.

[8] Fiona Crichton, George Dodd, Gian Schmid, Greg Gamble, and Keith J. Petrie. 2013. "Can Expectations Produce Symptoms From Infrasound Associated With Wind Turbines?." March 11. DOI: 10.1037/a0031760.

[9] Loren D. Knopper, and Christopher A. Ollson. "Health effects and wind turbines: A review of the literature." Environmental Health 10, no. 1 (2011): 78. DOI: 10.1186/1476-069X-10-78.

[10] T. Enk, K. Bay, M. Sonnenberg, J. Flaig, J. R. Boehrs, and A. Palochak. 2011. "Year 1 Post-Construction Avian and Bat Monitoring Report: Biglow Canyon Wind Farm Phase II, Sherman County, Oregon. September 10, 2009-September 12, 2010." Prepared for Portland General Electric Company, Portland, Oregon. Prepared by Western EcoSystems Technology, Inc.(WEST), Cheyenne, Wyoming, and Walla Walla, Washington. January 7. http://www.oregon.gov/energy/Siting/docs/BCW/BCW_2010Monitoring Report.pdf. 
[11] Benjamin K. Sovacool. 2009. "Contextualizing avian mortality: A preliminary appraisal of bird and bat fatalities from wind, fossil-fuel, and nuclear electricity." Energy Policy 37, no. 6: 2241-2248. DOI: 10.1016/j.enpol.2009.02.011.

[12] Wallace P. Erickson, Gregory D. Johnson, M. Dale Strickland, David P. Young Jr, Karyn J. Sernka, and Rhett E. Good. 2001. "Avian collisions with wind turbines: a summary of existing studies and comparisons to other sources of avian collision mortality in the United States." No. 1E. RESOLVE, Inc.(US). http://www.osti.gov/scitech/servlets/purl/822418

[13] Ajay Pal Singh Chabba. 2013. "Potential Environmental Impacts and Obstacles of Solar Energy." RESET: Smart Approaches to Sustainability. July 1. http://in.reset.org/blog/potential-environmental-impacts-andobstacles-solar-energy

[14] EERE, DOE; BLM, DOI. 2013. "Solar Energy Development Environmental Considerations." Solar Energy Development Programmatic EIS. September 3. http://solareis.anl.gov/guide/environment/index.cfm

[15] Good Company. 2008. "Life-Cycle Environmental Performance of Silicon Solar Panels." Oregon Department of Transportation. August. http://www.oregon.gov/ODOT/HWY/OIPP/docs/solar_panel_lifecycle.pdf

[16] Stuart Elmes. 2013. "Down to Earth - Will Scarce Rare Earths Impact the Solar Industry?" The Solarblogger. January 12. http://www.solarblogger.net/2013/01/down-to-earth-will-scarce-rareearths.html

[17] DOE. 2010. "Critical Materials Strategy." U.S. Department of Energy. December. http://energy.gov/sites/prod/files/edg/news/documents/critical materialsstrategy.pdf

[18] Department of Energy. 2012. "DOE-Funded Solar Variability Model in High Demand in Puerto Rico." SunShot Initiative High Penetration Solar Portal. October 10. https://solarhighpen.energy.gov/article/doe_funded_solar_variability_model high_demand_puerto_rico

[19] Ward T. Jewell and Timothy D. Unruh. "Limits on cloud-induced fluctuation in photovoltaic generation." Energy Conversion, IEEE Transactions on 5, no. 1 (1990): 8-14. DOI: 10.1109/60.50805.

[20] SI Staff. 2011. "Utilities Predict That Increased PV Deployment Will Pose Grid Challenges." Solar Industry. November 14. http://www.solarindustrymag.com/e107_plugins/content/content.php? content.9130\#.UQmUA67Z6PQ

[21] GE Energy. 2010 "Western wind and solar integration study." Prepared for The National Renewable Energy Laboratory. May. http://www.nrel.gov/docs/fy10osti/47781.pdf. Accessed at http://www.nrel.gov/electricity/transmission/western_wind.html

[22] David L. King, William E. Boyson, and Jay A. Kratochvil. 2002. "Analysis of factors influencing the annual energy production of photovoltaic systems." In Photovoltaic Specialists Conference, 2002. Conference Record of the TwentyNinth IEEE, pp. 1356-1361. IEEE. DOI: 10.1109/PVSC.2002.1190861. 
[23] S. Janjai, P. Pankaew, and J. Laksanaboonsong. 2009. "A model for calculating hourly global solar radiation from satellite data in the tropics." Applied Energy 86, no. 9: 1450-1457. DOI: 10.1016/j.apenergy.2009.02.005

[24] NOAA. 2013. "Solar Calculation Details." NOAA Earth System Research Laboratory: Global Monitoring Division. Accessed May 30. http://www.esrl.noaa.gov/gmd/grad/solcalc/calcdetails.html

[25] Wolfram Alpha LLC. 2013. "Day for Oahu, Hawaii, June 21, 2010." Wolfram|Alpha. Accessed June 1. http://www.wolframalpha.com/input/?i= Day+for+Oahu $\% 2 \mathrm{C}+$ Hawaii\%2C + June $+21 \% 2 \mathrm{C}+2010$

[26] J. Kolecki. 2008. "Calculating the Energy from Sunlight over an Actual Day." NASA Glen Research Center: Glenn Learning Technologies Project. Last modified December 8. http://www.grc.nasa.gov/WWW/k12/Numbers/Math/Mathematical_Thinking/sun12.htm

[27] C. Honsberg and Stuart Bowden. 2013. "Air Mass." PVEducation.org. Accessed May 5. http://pveducation.org/pvcdrom/properties-of-sunlight/airmass

[28] Mark Z. Jacobson. 2005. Fundamentals of Atmospheric Modeling (2 ed.). Cambridge University Press. p. 317. ISBN 0521548659.

[29] Dennis L. Hartmann. 1994. Global Physical Climatology. Academic Press. p. 30. ISBN 0080571638.

[30] Lawrence H. Auer and E. Myles Standish. 2000. "Astronomical refraction: computational method for all zenith angles." The Astronomical Journal 119, no. 5: 2472. DOI: $10.1086 / 301325$.

[31] Andrew T. Young. 1994. "Air mass and refraction." Applied optics 33, no. 6/2: 0. DOI: 10.1364/AO.33.001108.

[32] Lalit Kumar, Andrew K. Skidmore, and Edmund Knowles. 1997. "Modelling topographic variation in solar radiation in a GIS environment." International Journal of Geographical Information Science 11, no. 5: 475-497. DOI: $10.1080 / 136588197242266$.

[33] L. T. Wong and W. K. Chow. 2001. "Solar radiation model." Applied Energy 69, no. 3: 191-224. DOI: 10.1016/S0306-2619(01)00012-5.

[34] E. G. Laue. 1970. "The measurement of solar spectral irradiance at different terrestrial elevations." Solar Energy 13, no. 1: 43-57. DOI: 10.1016/0038092X(70)90006-X.

[35] Douglas V. Hoyt. 1978. "A model for the calculation of solar global insolation." Solar Energy 21, no. 1: 27-35. DOI: 10.1016/0038-092X(78)90113-5.

[36] Richard E. Bird and Carol Riordan. 1986. "Simple solar spectral model for direct and diffuse irradiance on horizontal and tilted planes at the earth's surface for cloudless atmospheres." Journal of Climate and Applied Meteorology 25, no. 1: 87-97. DOI: 10.1175/1520-0450(1986)025¡0087:SSSMFD ¿2.0.CO;2.

[37] Richard Perez, Robert Seals, Pierre Ineichen, Ronald Stewart, and David Menicucci. 1987. "A new simplified version of the Perez diffuse irradiance model for tilted surfaces." Solar energy 39, no. 3: 221-231. DOI: 10.1016/S0038-092X(87)80031-2. 
[38] Marcel Š́ri and Jaroslav Hofierka. 2004. "A New GIS?based Solar Radiation Model and Its Application to Photovoltaic Assessments." Transactions in GIS 8, no. 2: 175-190. DOI: 10.1111/j.1467-9671.2004.00174.x.

[39] Melih Yaramoglu, Russel B. Brinsfield, and Robert E. Muller Jr. 1985. "Estimation of solar radiation using stochastically generated cloud cover data." Energy in agriculture 4: 227-242. DOI: 10.1016/0167-5826(85)90019-1.

[40] Catherine Gautier, Georges Diak, and Serge Masse. 1980. "A simple physical model to estimate incident solar radiation at the surface from GOES satellite data." Journal of Applied Meteorology 19, no. 8: 1005-1012. DOI: 10.1175/1520-0450(1980)019\%3C1005:ASPMTE\%3E2.0.CO;2.

[41] Russell Brinsfield, Melih Yaramanoglu, and Fredrick Wheaton. 1984. "Ground level solar radiation prediction model including cloud cover effects." Solar energy 33, no. 6: 493-499. DOI: 10.1016/0038-092X(84)90003-3.

[42] Meca Solar. 2013. "Distinct Advantages." 2-AXIS Solar Trackers. Accessed June 5. http://www.mecasolar.com/_bin/ventajas_2_ejes.php

[43] A. K. Sinha, Venkataiah Mekala, and S. K. Samantaray. 2010. "Design and testing of PV maximum power tracking system with Matlab simulation." In Tencon 2010-2010 IEEE Region 10 Conference, pp. 466-473. IEEE. DOI: 10.1109/TENCON.2010.5686701.

[44] J. M. Gordon and Howard J. Wenger. 1991. "Central-station solar photovoltaic systems: Field layout, tracker, and array geometry sensitivity studies." Solar energy 46, no. 4: 211-217. DOI: 10.1016/0038-092X(91)90065-5.

[45] Sergio Vazquez, Srdjan M. Lukic, Eduardo Galvan, Leopoldo G. Franquelo, and Juan M. Carrasco. 2010. "Energy storage systems for transport and grid applications." Industrial Electronics, IEEE Transactions on 57, no. 12: 38813895. DOI: 10.1109/TIE.2010.2076414.

[46] G. Torres. 2006. "The Truth About NiCd Batteries." Last modified March 6, 2006. http://www.hardwaresecrets.com/article /The-Truth-About-NiCdBatteries/292/2

[47] ABB, Inc. 2013. "Worlds Largest Battery Energy Storage System." Accessed September 16. http://www05.abb.com/global/scot/scot232.nsf/veritydisplay/3c4e15816e4 a7bf1c12578d100500565/\$file/case_note_bess_gvea_fairbanks-web.pdf

[48] Golden Valley Electric Association. 2013. "Battery System." Accessed September 16. http://www.gvea.com/energy/bess

[49] W. B. Gu, C. Y. Wang, S. M. Li, M. M. Geng, and B. Y. Liaw. 1999. "Modeling discharge and charge characteristics of nickelmetal hydride batteries." Electrochimica Acta 44, no. 25: 4525-4541. DOI: 10.1016/S00134686(99)00187-5.

[50] Energizer. 2013. "Energizer NH15-2300." Form No. EBC - 7102WB. Accessed June 5. http://data.energizer.com/PDFs/nh15-2300.pdf

[51] David J. Bradwell, Hojong Kim, Aislinn HC Sirk, and Donald R. Sadoway. 2012. "MagnesiumAntimony Liquid Metal Battery for Stationary Energy Storage." Journal of the American Chemical Society 134, no. 4: 1895-1897. DOI: $10.1021 /$ ja209759s. 
[52] Ken Mattern, A. Ellis, S. E. Williams, C. Edwards, A. Nourai, and Dave Porter. 2008. "Application of inverter-based systems for peak shaving and reactive power management." In Transmission and Distribution Conference and Exposition, 2008. T\&D. IEEE/PES, pp. 1-4. IEEE. DOI: 10.1109/TDC.2008.4517244.

[53] Ambri. 2013. "Ambri Brochure." Accessed April 25. http://www.ambri.com/storage/documents/ambri-brochure.pdf

[54] Sandia National Laboratories. 2013. "Charleston Energy Storage Project." DOE International Energy Storage Database. Last modified April 1. http://www.energystorageexchange.org/projects/182

[55] William Tahil 2007. "The trouble with lithium." Implications of Future PHEV Production for Lithium Demand." January. Meridian International Research. http://www.inference.phy.cam.ac.uk/sustainable/refs/nuclear/Trouble Lithium.pdf

[56] Prachi Patel and Brian Perusse. 2012. "Grid battery storage gets big in the States." MRS bulletin 37, no. 11: 1004-1005. DOI: 10.1557/mrs.2012.294.

[57] Ludwig Joerissen, Juergen Garche, Ch Fabjan, and G. Tomazic. 2004. "Possible use of vanadium redox-flow batteries for energy storage in small grids and stand-alone photovoltaic systems." Journal of Power Sources 127, no. 1: 98-104. DOI: 10.1016/j.jpowsour.2003.09.066.

(starting at 58,59):

[58] E. Wesoff. 2011. GE's Gas-Fired Plants Could Enable More Wind and Solar Power. Greentech Media. May 25. http://www.greentechmedia.com/articles/read/ge-launching-gas-firedplants-better-suited-to-wind-and-solar-power

[59] Michael Milligan, Pearl Donohoo, Debra Lew, Erik Ela, Brendan Kirby, Hannele Holttinen, Eamonn Lannoye et al. (2010) "Operating reserves and wind power integration: an international comparison." In proc. 9th International Workshop on large-scale integration of wind power into power systems, pp. $18-29$.

[60] National Renewable Energy Laboratory. 2013. "Solar Measurement Grid (1Year Archive), 1-Second Global Horizontal Irradiance, Oahu, Hawaii." Measurement and Instrumentation Data Center (MIDC). Accessed May 30. DOI: $10.5439 / 1052451$

[61] LI-COR. 2013. "Pyranometer Light Sensors". Accessed July 1. http://www.licor.com/env/products/light/pyranometers/

[62] J. P. Kerr, G. W. Thurtell, and C. B. Tanner. 1967. "An Integrating Pyranometer for Climatological Observer Stations and Mesoscale Networks." Journal of Applied Meteorology 6: 688-694. DOI: 10.1175/15200450(1967)006;0688:AIPFCO ¿2.0.CO;2.

[63] C. A. Federer and C. B. Tanner. 1966. "Sensors for measuring light available for photosynthesis." Ecology: 654-657. Stable URL: http://www.jstor.org/stable/1933948. 
[64] NOAA. 2013 "1981-2010 Station Normals of Temperature, Precipitation, and Heating and Cooling Degree Days." National Climatic Data Center. Accessed September 23. http://www.ncdc.noaa.gov.

[65] D. G. Erbs, S. A. Klein, and J. A. Duffie. 1982. "Estimation of the diffuse radiation fraction for hourly, daily and monthly-average global radiation." Solar Energy 28, no. 4: 293-302. DOI: 10.1016/0038-092X(82)90302-4.

[66] J. F. Orgill and K. G. T. Hollands. 1977. "Correlation equation for hourly diffuse radiation on a horizontal surface." Solar energy 19, no. 4: 357-359. DOI: $10.1016 / 0038-092 X(77) 90006-8$.

[67] UO SRML. 2000. "YES Multifilter Rotating Shadowband Radiometer (MFR7)." University of Oregon, Solar Radiation Monitoring Laboratory. December 11. http://solardat.uoregon.edu/YESMFR.html

[68] Alexandre Oudalov, Daniel Chartouni, and Christian Ohler. 2007. "Optimizing a battery energy storage system for primary frequency control." Power Systems, IEEE Transactions on 22, no. 3: 1259-1266. DOI: 10.1109/TPWRS.2007.901459.

[69] R. Carbone. 2009. "Grid-connected photovoltaic systems with energy storage." In Clean Electrical Power, 2009 International Conference on, pp. 760767. IEEE. DOI: 10.1109/ICCEP.2009.5211967.

[70] U. Wilensky. 1999. "NetLogo." Center for Connected Learning and Computer-Based Modeling, Northwestern University, Evanston, IL. http://ccl.northwestern.edu/netlogo/.

[71] EIA. 2013. "Average Retail Price of Electricity to Ultimate Customers by End-Use Sector." U.S. Energy Information Administration. September 20. http://www.eia.gov/electricity/monthly/epm_table_grapher.cfm?t=epmt_5_6 $-\mathrm{a}$

[72] Matthew Lave and Jan Kleissl. 2012. "A Simple Cloud Simulator for Investigating the Correlation Scaling Coefficienct Used in the Wavelet Variability Model (WVM)." Paper presented at the World Renewable Energy Forum, Denver, Colorado. https://ases.conferenceservices.net/resources/252/2859/pdf/SOLAR2012_0726_full\%20paper.pdf

[73] J. St. John. 2013. "How Much Renewable Energy Can the Grid Handle?" Greentech Media. http://www.greentechmedia.com/articles/read/onthe-uncertain-edge-of-the-renewable-powered-grid

[74] R.W. Collins, A.S. Ferlauto, G.M. Ferreira, Chi Chen, Joohyun Koh, R.J. Koval, Yeeheng Lee, J.M. Pearce, C.R. Wronski. 2008. "Evolution of microstructure and phase in amorphous, protocrystalline, and microcrystalline silicon studied by real time spectroscopic ellipsometry." Solar Energy Materials and Solar Cells. Volume 78, Issues 14, July. Pages 143-180. ISSN 0927-0248. DOI: http://dx.doi.org/10.1016/S0927-0248(02)00436-1.

[75] S. M. Chalmers, M. M. Hitt, J. T. Underhill, P. M. Anderson, P. L. Vogt, and R. Ingersoll. 1985. "The effect of photovoltaic power generation on utility operation." Power Apparatus and Systems, IEEE Transactions on 3: 524-530. DOI: 10.1109/TPAS.1985.318968. 
[76] Charles J. Barnhart, Michael Dale, Adam R. Brandt, and Sally M. Benson. 2013. "The energetic implications of curtailing versus storing solarand wind-generated electricity." Energy \& Environmental Science. DOI: $10.1039 / \mathrm{c} 3 \mathrm{ee} 41973 \mathrm{~h}$.

[77] Fritz Kasten, and Andrew T. Young. 1989. "Revised optical air mass tables and approximation formula." Appl. Opt 28, no. 22: 4735-4738. DOI: 10.1364/AO.28.004735.

[78] C. Monteiro, R. Bessa, V. Miranda, A. Botterud, J. Wang, and G. Conzelmann. 2009. "Wind power forecasting: state-of-the-art 2009." No. ANL/DIS10-1. Argonne National Laboratory (ANL). DOI: 10.2172/968212.

[79] First Solar. 2013. "The First "Global and Comprehensive Module Recycling Program." Accessed July 5. http://www.firstsolar.com/Sustainability/Environmental/Product-lifecycle-management/Recycling-Process

[80] Kadra Branker, M. J. M. Pathak, and J. M. Pearce. 2011. "A review of solar photovoltaic levelized cost of electricity." Renewable and Sustainable Energy Reviews 15, no. 9: 4470-4482. DOI: 10.1016/j.rser.2011.07.104.

[81] NERC. 2009. "Accommodating High Levels of Variable Generation." North American Electric Reliability Corporation (NERC). April. http://www.nerc.com/files/ivgtf_report_041609.pdf

[82] PGE. 2013. "2012 Annual Report." Portland General Electric Company. Accessed April 28. http://investors.portlandgeneral.com/annuals.cfm

[83] Cody A. Hill, Matthew Clayton Such, Dongmei Chen, Juan Gonzalez, and W. Mack Grady. 2012. "Battery energy storage for enabling integration of distributed solar power generation." Smart Grid, IEEE Transactions on 3, no. 2: 850-857. DOI: 10.1109/TSG.2012.2190113.

[84] FERC. 2013. "Electric Quarterly Reports (EQR)." Federal Energy Regulatory Commision (FERC). Accessed June 1. http://www.ferc.gov/docsfiling/eqr.asp\#.Uj6ixRZ8xFI

[85] Zhenguo Yang, Jianlu Zhang, Michael CW Kintner-Meyer, Xiaochuan Lu, Daiwon Choi, John P. Lemmon, and Jun Liu. 2011. "Electrochemical energy storage for green grid." Chemical Reviews 111, no. 5: 3577-3613. DOI: $10.1021 / \mathrm{cr} 100290 \mathrm{v}$.

[86] KEMA. 2013. "Energy Storage for System Reliability and Interconnection." DNV KEMA. Accessed June 6. http://www.dnvkema.com/services/etd/es/default.aspx

[87] Richard E. Brown. 2008. "Impact of Smart Grid on distribution system design." In Power and Energy Society General Meeting-Conversion and Delivery of Electrical Energy in the 21st Century, 2008 IEEE, pp. 1-4. IEEE. DOI: 10.1109/PES.2008.4596843.

[88] Douglas Backer. 2007. "Power Quality and Asset Management The Other" Two-Thirds" of AMI Value." In 2007 IEEE Rural Electric Power Conference, Rapid City, SD. DOI: 10.1109/REPCON.2007.369560. 
[89] R. Ray. 2013. "Sandy Creek Coal-fired Power Plant Set to Go Online in April." Power Engineering. February 15. http://www.powereng.com/articles/2013/02/sandy-creek-coal-fired-power-plant-set-to-goonline-in-april.html

[90] Hassan Farhangi. 2010. "The path of the smart grid." Power and Energy Magazine, IEEE 8, no. 1: 18-28. DOI: 10.1109/MPE.2009.934876.

[91] NPR. 2009. "Visualizing the U.S. Electric Grid." National Public Radio (NPR). April 24. http://www.npr.org/templates/story/story.php?storyId=110997398

[92] Trung Nguyen and Robert F. Savinell. 2010. "Flow batteries." Electrochemical Society Interface 19, no. 3: 54-56. http://www.electrochem.org/dl/interface/fal/fal10/fal10_p054-056.pdf.

[93] Jim Eyer and Garth Corey. 2010. "Energy storage for the electricity grid: Benefits and market potential assessment guide." Sandia National Laboratories Report, SAND2010-0815, Albuquerque, New Mexico. http://www.smartgrid.gov/sites/default/files/resources/energy_storage.pdf.

[94] K. C. Divya and Jacob Østergaard. 2009. "Battery energy storage technology for power systemsAn overview." Electric Power Systems Research 79, no. 4: 511-520. DOI: 10.1016/j.epsr.2008.09.017.

[95] Claude Solnik. 2011. "LIPA Eyes World's Biggest Battery." Long Island Business News September 21. http://www.aesenergystorage.com/news/libn-lipaeyes-worlds-biggest-battery.html

[96] M. Paulescu, and Z. Schlett. 2003. "A simplified but accurate spectral solar irradiance model." Theoretical and applied climatology 75, no. 3-4: 203-212. DOI: $10.1007 / \mathrm{s} 00704-003-0731-y$.

[97] Christian A. Gueymard. 2001. "Parameterized transmittance model for direct beam and circumsolar spectral irradiance." Solar Energy 71, no. 5: 325-346. DOI: $10.1016 / \mathrm{S} 0038-092 \mathrm{X}(01) 00054-8$.

[98] Alex Cronin. 2009. "Brief report on NAS storage batteries." February 20. http://www.physics.arizona.edu/ cronin/Solar/References/Storage/

[99] Lisa Zyga. 2013. "Sodium-ion battery cathode has highest energy density to date." Phys.org. September 12. http://phys.org/news/2013-09-sodium-ionbattery-cathode-highest-energy.html

[100] Verónica Palomares, Paula Serras, Irune Villaluenga, Karina B. Hueso, Javier Carretero-González, and Teófilo Rojo. 2012. "Na-ion batteries, recent advances and present challenges to become low cost energy storage systems." Energy \& Environmental Science 5, no. 3: 5884-5901. DOI: 10.1039/C2EE02781J.

[101] Haitao Zhuo, Xianyou Wang, Anping Tang, Zhiming Liu, Sergio Gamboa, and P. J. Sebastian. 2006. "The preparation of NaV1-xCrxPO4F cathode materials for sodium-ion battery." Journal of Power Sources 160, no. 1: 698703. DOI: $10.1016 /$ j.jpowsour.2005.12.079.

[102] Green Car Congress. 2012. "MIT team calls initial performance results of magnesium-antimony liquid metal battery 'promising'." January 23. http://www.greencarcongress.com/2012/01/sadoway-20120123.html 
[103] PV Depot. 2013 "Hanwha 285 Watt HSL72P6-PA-0-285T Solar Panel." Accessed September 1. http://pvdepot.com/hanwha-285-watt-hsl72p6-pa-0285t-solar-panel.html

[104] John Whitney, Jr. 2012. "Levelized Cost of Energy: Renewable energy has achieved parity." CleanEnergy Action Project Blog. June 18. http://www.cleanenergyactionproject.com/CleanEnergyActionProject/Blog /Entries/2012/6/18_Levelized_Cost_of_Energy__Renewable_energy_has_ achieved_parity.html

[105] CleanEnergy. Project

2013.

"Agua

Caliente

Solar http://www.cleanenergyactionproject.com/CleanEnergyActionProject/CS. Agua_Caliente_Solar_Project__-_Thin_Film_Photovoltaic_Solar_Power_Case_ Studies.html

[106] SunPower Corp. 2010. "E19 / 425 SOLAR PANEL." March. http://us.sunpowercorp.com/cs/Satellite?blobcol=urldata\&blobheader $=$ application\%2Fpdf\&blobheadername3=Content-Disposition\&blobheader value $3=$ attachment $\% 3 \mathrm{~B}+$ filename\%3D615\%252F177\%252FSP425\%2BPanel \%2BSpec\%2BSheet\%2Bfor\%2BDownload\%252C1.pdf\&blobkey=id\&blob table $=$ MungoBlobs\&blobwhere $=1300258501532 \&$ ssbinary $=$ true

[107] D. Kottick, M. Blau, and D. Edelstein. 1993. "Battery energy storage for frequency regulation in an island power system." IEEE Transactions on Energy Conversion 8, no. 3: 455-459. DOI: 10.1109/60.257059.

[108] M. K. C. Marwali, Ma Haili, S. M. Shahidehpour, and K. H. Abdul-Rahman. 1998. "Short term generation scheduling in photovoltaic-utility grid with battery storage." IEEE Transactions on Power Systems 13, no. 3: 1057-1062. DOI: $10.1109 / 59.709099$.

[109] Francois Giraud, and Ziyad M. Salameh. 1999. "Analysis of the effects of a passing cloud on a grid-interactive photovoltaic system with battery storage using neural networks." IEEE Transactions on Energy Conversion 14, no. 4: 1572-1577. DOI: 10.1109/60.815107.

[110] Bruce Dunn, Haresh Kamath, and Jean-Marie Tarascon. 2011. "Electrical energy storage for the grid: A battery of choices." Science 334, no. 6058: 928-935. DOI: 10.1126/science.1212741.

[111] Peter J. Hall, and Euan J. Bain. 2008. "Energy-storage technologies and electricity generation." Energy policy 36, no. 12: 4352-4355. DOI: 10.1016/j.enpol.2008.09.037. 


\section{Appendix A: Supplemental Data}

\section{A.1 Energy Price per kWh}

While the power supplier would almost certainly get less than the retail cost of energy for the energy they sell to a utility, this approximation is not as liberal as it might seem. The price per $\mathrm{kWh}$ included in the model is the national average for the month of July, 2013 across all sectors [71]. This price is an approximation of the entire return on investment for the project developer, which would include not only the price they earned from selling power, but also government subsidies — which depending on the municipality of the project can make up a significant portion of the return on the investment.

Furthermore, many utilities, (including Portland General Electric in Oregon), offer their customers the opportunity to pay an additional cost per kWh on top of the base electricity rate to ensure that their demand is met exclusively by renewables such as wind, solar, and hydro. This allows the utilities to offer a higher price for that energy to the producer, to ensure that they have adequate supply to be able to make that renewable guarantee.

\section{A.2 Initial Model Versions}

The multiple initial attempts at formulating a model were not the result of my specifically manipulating the individual parameters of the algorithms, but rather because of trying various formulae to simulate the direct and diffuse irradiance. These formulae were from a number of different sources which were each based largely on empirical statistical fits of data collected at different times, with differ- 
ent conditions, and different technologies.

Earlier in the research, I tried the formula for $I_{D i r N}$ given by

$$
\left.I_{\text {DirN }}=1.353 \cdot(1-a \cdot h) \cdot 0.7^{\text {AirMass }^{0.678}}+a \cdot h\right) \cdot \cos \left(\phi-\delta_{\odot}\right)
$$

which is an empirical fit to data observed at several terrestrial sites — with $a$ being a constant equal to 0.14 , and $h$ being equal to the elevation of the site above sea level in kilometers [27]. However, while this equation gave easily generalizable results across a broad spectrum of cases, it was not sufficiently accurate for the purposes of this thesis.

\section{A.3 Preliminary Results}

Figure A.1 show some preliminary results of the model prior to introducing the effects of "diffuse peaks" and absolute leveling. One of the primary negative aspects of this iteration of the model was that it unnecessarily kept the battery charge state nearly constant throughout the day, while allowing the output to vary within a certain threshold.

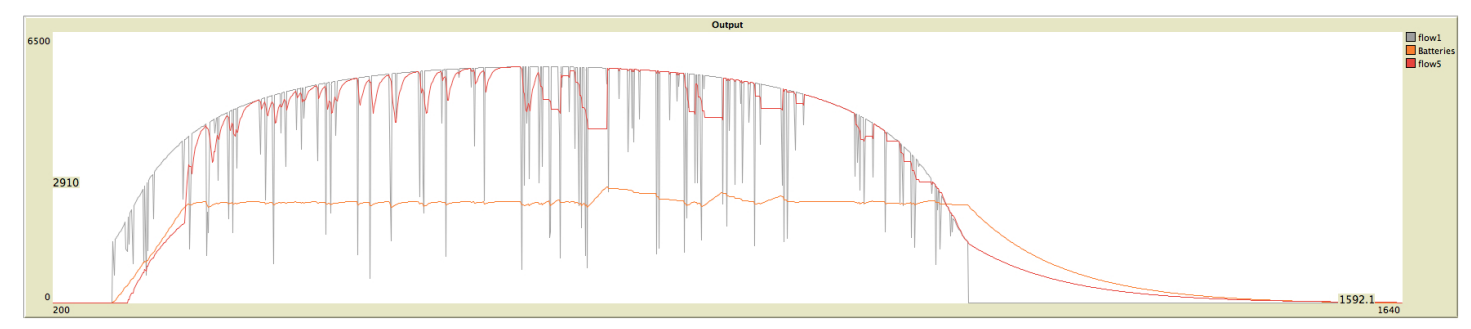

Figure A.1: Some preliminary results of output of $5.7 \mathrm{MW}$ PV project with 3 MWh capacity BESS

\section{A.4 Experiment 5 Parts I \& II}

The results from Experiment 5, part I (Ni-Cd only), and part II (Li-Ion only) are included below in Table A.1 and Table A.2, respectively. 


\begin{tabular}{lrrrrrrr}
\hline & & Value & Run \# & Batt_Type & Capacity & SS_Charge & Lvl_Tar \\
\hline \multirow{7}{*}{ VRF } & 1st & 0.9972 & 2486 & "Ni-Cd" & 0.3 & 0.575 & 0.7 \\
& 2nd & 0.9971 & 9290 & "Ni-Cd" & 0.6 & 0.575 & 0.7 \\
& 3rd & 0.9969 & 9857 & "Ni-Cd" & 0.625 & 0.575 & 0.7 \\
\multirow{2}{*}{ R_Ad } & 4th & 0.9967 & 3620 & "Ni-Cd" & 0.35 & 0.575 & 0.7 \\
& 1st & 2212 & 9360 & "Ni-Cd" & 0.6 & 0.6 & 0.725 \\
& 2nd & 2211 & 9668 & "Ni-Cd" & 0.625 & 0.5 & 0.7 \\
& 3rd & 2211 & 6833 & "Ni-Cd" & 0.5 & 0.5 & 0.7 \\
\multirow{2}{*}{ R_Tot } & 4th & 2211 & 596 & "Ni-Cd" & 0.225 & 0.5 & 0.7 \\
& 1st & 615.1 & 6833 & "Ni-Cd" & 0.5 & 0.5 & 0.7 \\
& 2nd & 614.9 & 9668 & "Ni-Cd" & 0.625 & 0.5 & 0.7 \\
& 3rd & 614.5 & 4565 & "Ni-Cd" & 0.4 & 0.5 & 0.7 \\
& 4th & 614.2 & 596 & "Ni-Cd" & 0.225 & 0.5 & 0.7 \\
\hline
\end{tabular}

Table A.1: Reasonable configurations from BehaviorSpace Experiment 5, part 1/ 2

\begin{tabular}{rrrrrrrr}
\hline & & Value & Run \# & Batt_Type & Capacity & SS_Charge & Lvl_Tar \\
\hline \multirow{6}{*}{ VRF } & 1st & 0.9941 & 7085 & "Li-Ion" & 0.5 & 0.6 & 0.7 \\
& 2nd & 0.9900 & 12755 & "Li-Ion" & 0.75 & 0.6 & 0.7 \\
& 3rd & 0.9899 & 5447 & "Li-Ion" & 0.425 & 0.625 & 0.7 \\
\multirow{6}{*}{ R_Ad } & 4th & 0.9891 & 7344 & "Li-Ion" & 0.5 & 0.7 & 0.725 \\
& 1st & 2268 & 7085 & "Li-Ion" & 0.5 & 0.6 & 0.7 \\
& 2nd & 2265 & 7344 & "Li-Ion" & 0.5 & 0.7 & 0.725 \\
& 3rd & 2263 & 10746 & "Li-Ion" & 0.65 & 0.7 & 0.7 \\
\multirow{2}{*}{ R_Tot } & 4th & 2262 & 12755 & "Li-Ion" & 0.75 & 0.6 & 0.7 \\
& 1st & 679.6 & 7085 & "Li-Ion" & 0.5 & 0.6 & 0.7 \\
& 2nd & 671.2 & 12755 & "Li-Ion" & 0.75 & 0.6 & 0.7 \\
& 3rd & 670.9 & 7344 & "Li-Ion" & 0.5 & 0.7 & 0.725 \\
& 4th & 669.4 & 10746 & "Li-Ion" & 0.65 & 0.7 & 0.7 \\
\hline
\end{tabular}

Table A.2: Reasonable configurations from BehaviorSpace Experiment 5, part 2/ 2 


\section{Appendix B: Data Processing}

\section{B.1 FileIO Script}

The $\mathrm{C}++$ script that I used to find the comparative metrics on the years worth of MIDC second-to-second timescale irradiance data from the Oahu site is included below.

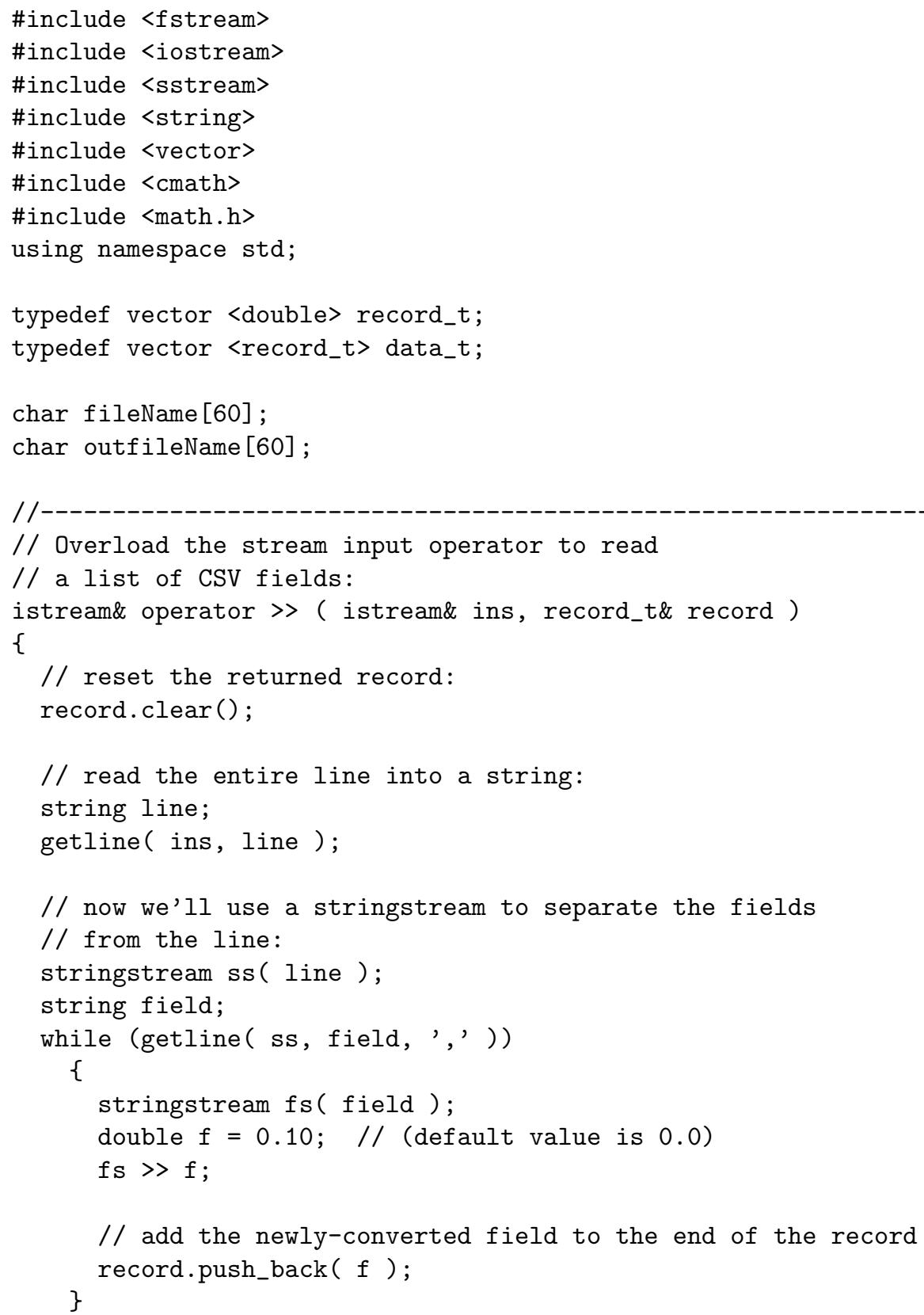




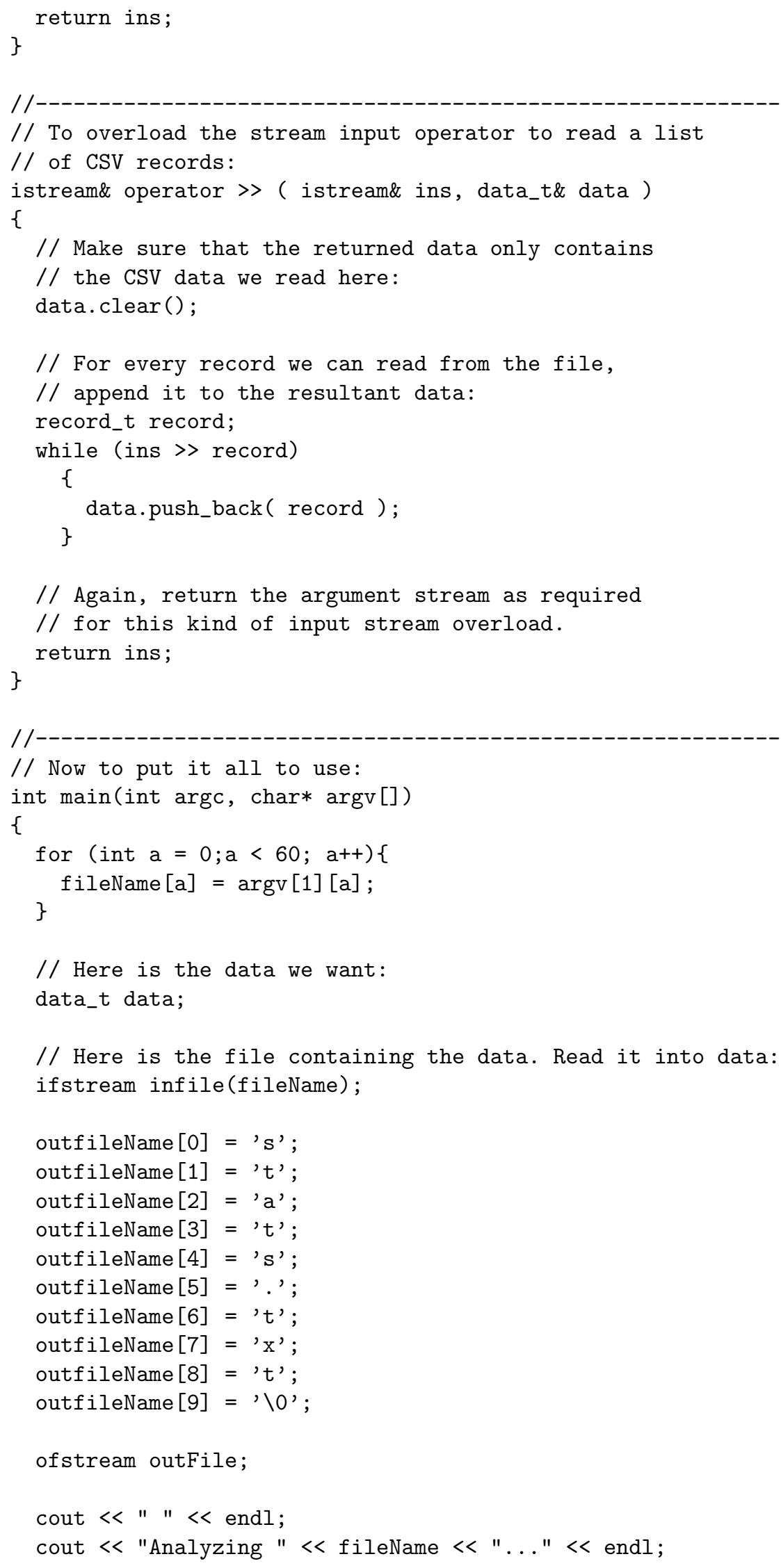




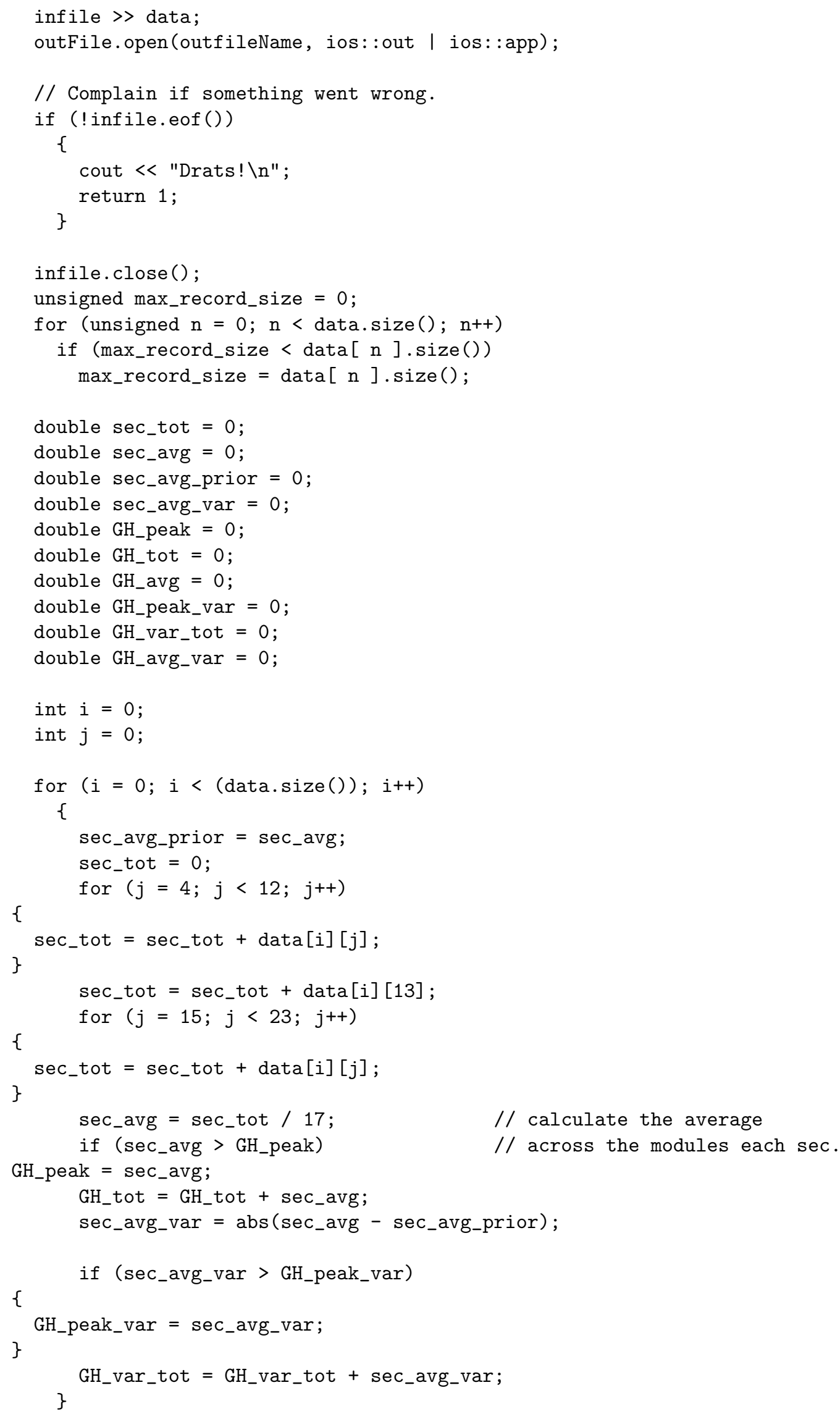




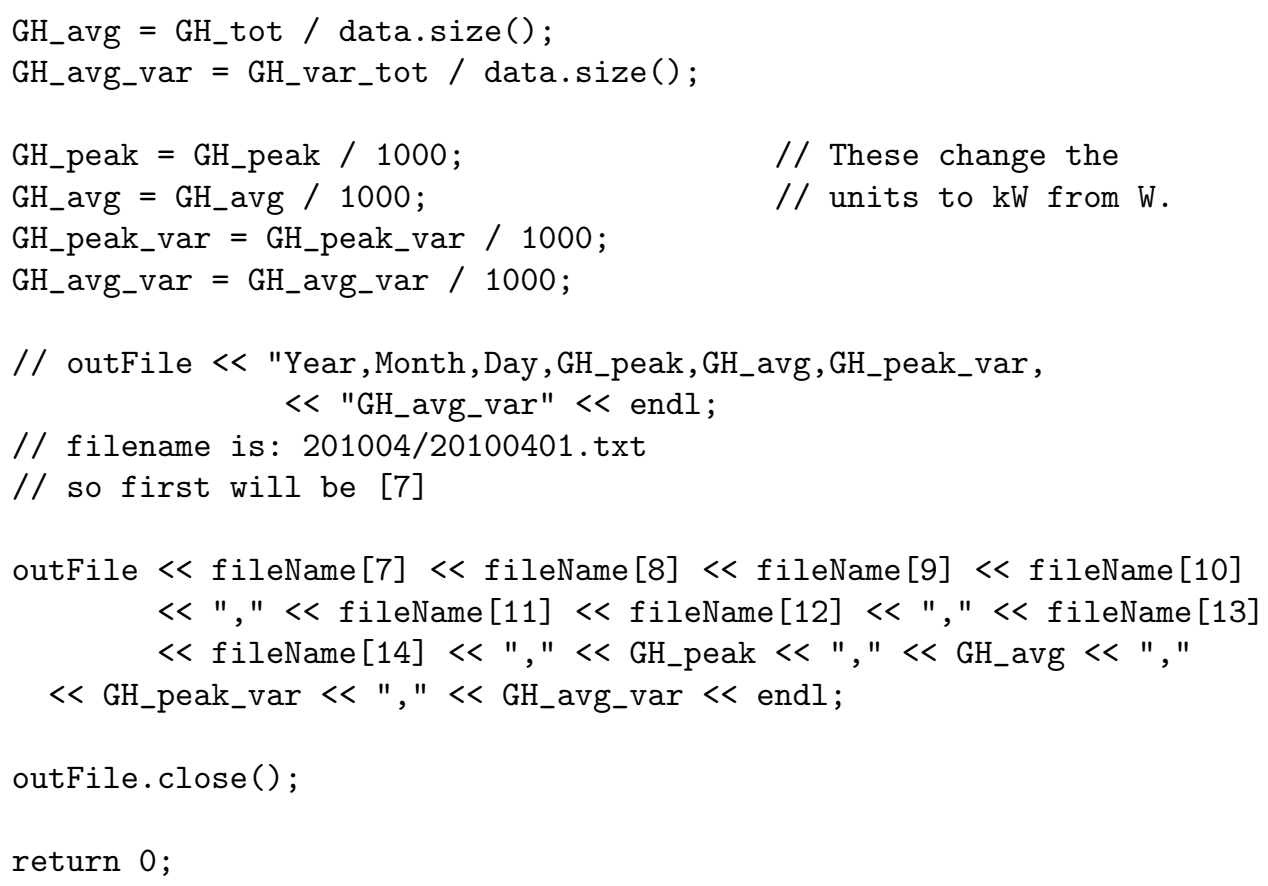

\section{B.2 Shell Script}

I automated the execution of the FileIO script on the 365 MIDC files from Oahu using the following Shell script:

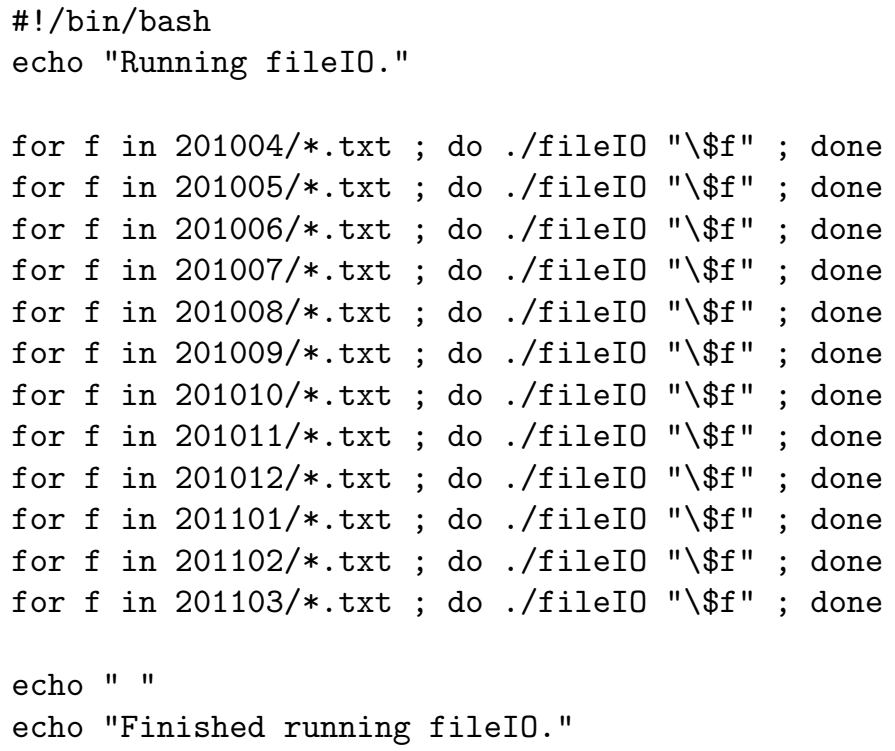

